\title{
The conjugacy problem for Coxeter groups
}

\author{
Daan Krammer
}

\begin{abstract}
We prove that for every finite rank Coxeter group there exists a polynomial (cubic) solution to the conjugacy problem.
\end{abstract}

Mathematics Subject Classification (2000). 20F55, 20F10, 22E40.

Keywords. Coxeter group, conjugacy problem, Tits cone, parabolic closure.

\section{Contents}

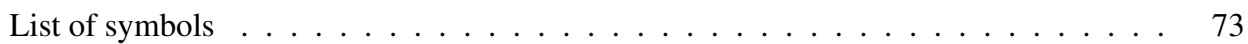

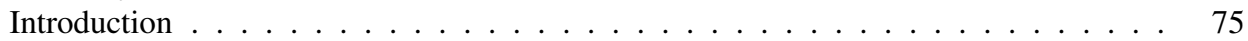

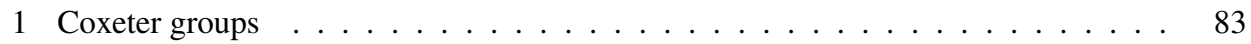

1.1 Coxeter groups $\ldots \ldots \ldots \ldots \ldots \ldots$

1.2 Root bases . . . . . . . . . . . . . . . . . . . 84

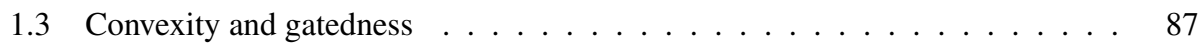

1.4 Reflection subgroups . . . . . . . . . . . . . . . . . . . . . . . 89

1.5 Nonnegative matrices . . . . . . . . . . . . . . . . . . . . . . 92

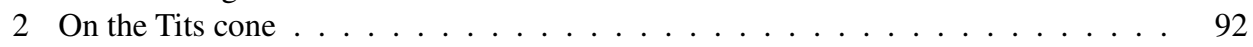

2.1 The Galois correspondence . . . . . . . . . . . . . . . . . 93

2.2 The interior of the Tits cone . . . . . . . . . . . . . . . . 95

3 The normalizer of a parabolic subgroup . . . . . . . . . . . . . . . . 97

3.1 The normalizer of a parabolic subgroup . . . . . . . . . . . . . . . 97

3.2 Finite subgroups of Coxeter groups . . . . . . . . . . . . . . . . 101

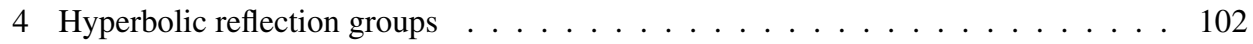

4.1 Signatures . . . . . . . . . . . . . . . . . . . . . 103

4.2 Affine Coxeter groups . . . . . . . . . . . . . . . . . . . . . 103

4.3 Hyperbolicity and word hyperbolicity . . . . . . . . . . . . . . 104

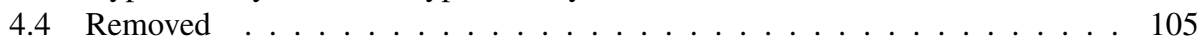

4.5 Hyperbolic space . . . . . . . . . . . . . . . . . . . 105

4.6 Hyperbolic reflection groups . . . . . . . . . . . . . . . . . . 106

4.7 The conjugacy problem . . . . . . . . . . . . . . . . 107

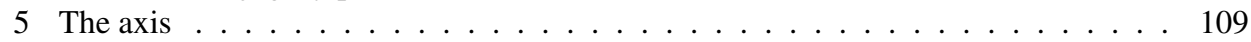

5.1 Introduction . . . . . . . . . . . . . . . . 109 
5.2 Periodic, even and odd roots . . . . . . . . . . . . . . 110

5.3 Distinguishing even roots from odd ones . . . . . . . . . . . . . . . . . . . . 112

5.4 A pseudometric on $U^{0} \ldots \ldots \ldots \ldots \ldots \ldots \ldots \ldots$

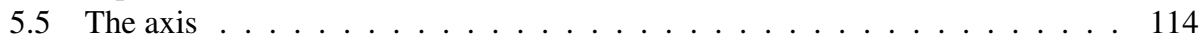

5.6 Non-emptiness of the axis . . . . . . . . . . . . . . . . . 120

5.7 Critical roots . . . . . . . . . . . . . . . . . . . . . . . . . 122

5.8 The parabolic closure . . . . . . . . . . . . . . . . . . . . . . 127

5.9 Calculations in number fields . . . . . . . . . . . . . . . . . . . . 129

5.10 Algorithms . . . . . . . . . . . . . . . . . . . . . . . . . . . . . . . . . 130

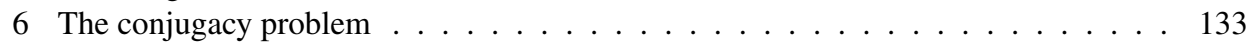

6.1 Dividing out the radical . . . . . . . . . . . . . . . . . 133

6.2 Ordered triples of roots . . . . . . . . . . . . . . . . . . 134

6.3 Outward roots and their ordering . . . . . . . . . . . . . . 136

6.4 First solution to the conjugacy problem . . . . . . . . . . . . . . . . . . 140

6.5 The greatest eigenvalue . . . . . . . . . . . . . . . . . . . . . . . . . . . . . . . . 144

6.6 Removed . . . . . . . . . . . . . . . . . . 151

6.7 Second solution to the conjugacy problem . . . . . . . . . . . . . 151

6.8 Free abelian groups in Coxeter groups . . . . . . . . . . . . . . . . . 153

Appendix A Euclidean complexes of non-positive curvature . . . . . . . . . . 155

A.1 Metric spaces . . . . . . . . . . . . . . . . . . 155

A.2 Metric complexes . . . . . . . . . . . . . . . . . 156

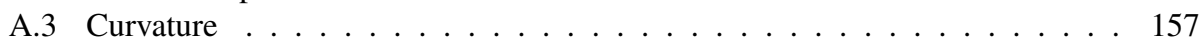

A.4 Convex metrics . . . . . . . . . . . . . . . . . . 158

Appendix B The Davis-Moussong complex . . . . . . . . . . . . . . . . . . . . 159

B.1 Nerves of almost negative matrices . . . . . . . . . . . . . . . . 159

B.2 Cells for the Davis-Moussong complex . . . . . . . . . . . . . . . 160

B.3 The Davis-Moussong complex . . . . . . . . . . . . . . . . . 161

B.4 The embedding of the Davis-Moussong complex into the Tits cone . . . . . . 163

B.5 Comparison of $d_{W}$ and $d_{M} \ldots \ldots \ldots \ldots \ldots \ldots \ldots$

B.6 The conjugacy problem . . . . . . . . . . . . . . . . 167

References . . . . . . . . . . . . . . . . . . 168 


\section{List of symbols}

\begin{tabular}{|c|c|c|}
\hline \multirow{4}{*}{$\begin{array}{l}a_{s}, 160 \\
a_{s t}=\left(e_{s}, e_{t}\right), 85 \\
A_{I}, \text { Gram matrix, } 85 \\
A(\alpha), \text { halfspace, } 86\end{array}$} & $K_{I}, 94$ & $S_{J}, 159$ \\
\hline & $K(\alpha), K(A), 91$ & \multirow{3}{*}{$\begin{array}{l}U, \text { Tits cone, } 85 \\
U^{0}, \text { interior of } U, 85\end{array}$} \\
\hline & $K, 97$ & \\
\hline & $\ell, 84,104$ & \\
\hline$c, 112$ & $L(w), 153$ & $w_{I}$, longest element, 87 \\
\hline$C$, fundamental chamber, & Lk, link, 157 & $\begin{array}{l}w_{p}, 162 \\
W\end{array}$ \\
\hline$C_{I}, 85$ & $m_{s t}$, order of $s t, 83$ & $W_{I}, 84$ \\
\hline$C(w)$, centralizer, 150 & $\begin{array}{l}m(w), 152 \\
M=M(W), 162\end{array}$ & $\bar{x}$ sunnort 156 \\
\hline Cp, complement, 157 & $\begin{array}{l}M=M(W), 102 \\
M_{0}(w), 152\end{array}$ & $\begin{array}{l}x, \text { support, } 150 \\
x_{J}, X_{J}, 160\end{array}$ \\
\hline $\begin{array}{l}d, \text { metric on } H^{n}, 105 \\
d, \text { metric on a complex, } \\
157\end{array}$ & $\begin{array}{l}N\left(W_{I}\right) \text {, normalizer, } 99 \\
N(A), \text { nerve, } 159\end{array}$ & $\begin{array}{l}X^{0}, \text { interior of a cell, } \\
156 \\
X_{J K}, 160\end{array}$ \\
\hline $\begin{array}{l}\operatorname{deg}(\lambda, w), \operatorname{deg}(w), 144 \\
\operatorname{deg}\left(\lambda, a_{n}\right), \operatorname{deg}\left(a_{n}\right), 145\end{array}$ & $\begin{array}{l}\operatorname{Ost}(x), \text { open star, } 156 \\
\operatorname{Out}(w), 119\end{array}$ & $Y_{J}, 162$ \\
\hline $\begin{array}{l}d_{W}, \text { metric on } W, 84 \\
d_{\alpha}, d_{\Phi}, 113\end{array}$ & $\mathrm{O}^{+}(n, 1), 105$ & $Z(w)$, centralizer, 115 \\
\hline$D, D_{I}, 107$ & $p_{\mu}, p_{w}, 144$ & $Z\left(W_{I}\right)$, centralizer, 99 \\
\hline$D_{I J}, 88$ & $\begin{array}{l}p_{I}, 95 \\
P[X], 122\end{array}$ & $\lambda=\rho(w), 144$ \\
\hline$e_{s}, 85$ & $\operatorname{Pc}(X)$, parabolic closure, & $\lambda(e), 98$ \\
\hline $\begin{array}{l}E, 84 \\
E^{*} 84\end{array}$ & 94 & $\mu(\alpha)$, wall, 93 \\
\hline $\begin{array}{l}E^{4}, 84 \\
E_{I}, 85\end{array}$ & $\begin{array}{l}\mathrm{Pc}^{\infty}(w), \mathrm{Pc}^{0}(w), 128 \\
P S^{n}, 105\end{array}$ & $\mu(A)$, wall, 114 \\
\hline $\begin{array}{l}E_{\mu}, E_{w}, 144 \\
E_{>0}, 120\end{array}$ & 10 & $v(I, s), 97$ \\
\hline $\mathbb{E}^{n}$, Euclidean space, 156 & $Q(w)$, axis, 114 & $\pi, 104,105$ \\
\hline $\begin{array}{l}f_{s}, 85 \\
f_{S}^{J}, 160\end{array}$ & $\begin{array}{l}Q_{0}(w), 150 \\
Q_{1}(w), Q_{2}(w), 120 \\
Q^{*}(w), 126\end{array}$ & $\begin{array}{l}\Pi, 84 \\
\Pi_{I}, 85\end{array}$ \\
\hline$f_{\alpha}, 113$ & $Q^{+}(w), 129$ & $\rho(w), 144$ \\
\hline$F(X)$, free group, 75 & $Q(x)=(x, x), 105$ & $\rho\left(a_{n}\right), 145$ \\
\hline $\begin{array}{l}g_{J}^{K}, 160 \\
G_{J}, 99\end{array}$ & $r=\# S, 112$ & $\phi, 99,104,107$ \\
\hline & $\operatorname{rk}(G), 153$ & $\Phi$, root system, 86 \\
\hline$H, 105$ & $r(w), 119$ & $\Phi_{I}, 86$ \\
\hline$H^{+}, H^{-}, 105$ & $r_{\alpha}$, reflection, 85,86 & $\Phi_{\text {odd }}, 128$ \\
\hline $\begin{array}{l}\mathbb{H}^{n} \text {, hyperbolic space, } \\
105\end{array}$ & $\begin{array}{l}r_{A}, 86 \\
R_{g}, 107\end{array}$ & $\psi, 99$ \\
\hline$H(\alpha), H(A), 91$ & $S$, generators of $W, 85$ & $(\cdot, \cdot), 84$ \\
\hline$I^{\perp}, 90$ & $S, S^{+}, S^{-}, S^{n-1}, 105$ & {$[x, y], 85$} \\
\hline$I \stackrel{s}{\longrightarrow} J, 97$ & $S(\lambda)$, circle, 158 & $<$, ordering on roots, 91 \\
\hline$J(p), 162$ & $\operatorname{St}(x)$, star, 156 & $*, 158$ \\
\hline
\end{tabular}




\section{Dependence of sections and appendices}

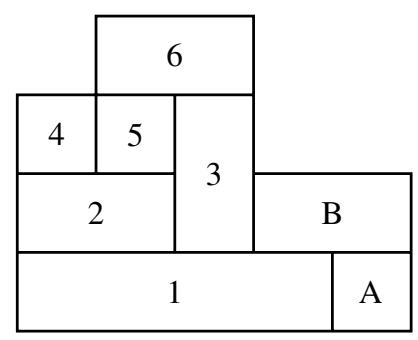

\section{Notational conventions}

$\begin{array}{ll}\# X & \text { cardinality of a set } X \\ \langle X\rangle & \text { group or root system generated by a set } X \\ w^{\mathbb{Z}} & \text { group generated by an element } w \\ \backslash & \text { division by a left group action } \\ - & \text { set difference } \\ \subset & \text { (not necessarily strict) inclusion } \\ \sqcup & \text { disjoint union } \\ X^{0} & \text { topological interior of } X \\ \bar{X} & \text { topological closure of } X \\ C(w) & \text { conjugacy class } \\ N(H) & \text { normalizer } \\ Z(H) & \text { centralizer } \\ G \ltimes N & \text { semi-direct product with normal subgroup } N \\ \text { Ann } & \text { annihilator for dual vector spaces } \\ \mathrm{Ch} & \text { convex hull } \\ \mathrm{Im} & \text { image of a map } \\ \mathrm{O}(k), \mathrm{O}(k, \ell) & \text { real orthogonal groups } \\ P(X) & \text { power set of } X \\ \mathrm{Span} & \text { linear span } \\ \mathrm{Sym}(X) & \text { symmetric group on } X \\ & \end{array}$




\section{Introduction}

Algorithms. There exists a precise definition of what an algorithm is ([40], Chapter 5), but for reading this paper it is enough to have as little familiarity with the concept as one acquires after writing a few computer programs.

More important to us than whether an algorithm exists is whether a fast algorithm exists: the complexity. An algorithm is called polynomial of exponent $k$ if $t(n)=$ $\mathcal{O}\left(n^{k}\right)$ where $t(n)$ is the maximal time taken by the algorithm on input of length $n>0$. An algorithm is called exponential if we have $t(n)=\mathcal{O}\left(A^{n}\right)$ for some $A>1$.

These are upper bounds for $t(n)$. If we are interested in lower bounds, we say so explicitly.

Algorithmic problems for groups. In 1912, Max Dehn [26] stated, and in certain cases solved, three fundamental problems on groups, two of which are as follows. Let $G=\langle X \mid R\rangle$ be a (say finitely) presented group. Thus, $X$ is a finite set, and $R$ is a finite subset of the free group $F(X)$ on $X$, and $G=F(X) / N$, where $N$ is the smallest normal subgroup of $F(X)$ containing $R$. Let $\pi: F(X) \rightarrow G$ denote the natural map.

Word problem: Does there exist an algorithm that, given $w \in F(X)$, decides whether or not $\pi w=1$ ?

Conjugacy problem: Does there exist an algorithm that, given $v, w \in$ $F(X)$, decides whether or not $\pi v$ and $\pi w$ are conjugate in $G$ ?

If an algorithm for the word problem exists, we say that the group has solvable word problem, and similarly for the conjugacy problem.

Note that if the conjugacy problem for a certain group is solvable, then so is the word problem. It is easy to see that the solvability of the two problems does not depend on the given presentation, only on the group $G$. For a survey on the conjugacy problem, we refer to [37].

In order to make the complexity of the Dehn problems well defined, we need to define what their input lengths are, at least up to reparametrisation by a polynomial.

Let $\ell: F(X) \rightarrow \mathbb{Z}$ be the length with respect to $X$. We saw that the input of the word problem is an element $g \in F(X)$; the length of this input is defined to be $\ell(g)$. Likewise, the input of the conjugacy problem is a pair $(g, h) \in F(X) \times F(X)$ and we define its length to be $\ell(g)+\ell(h)$.

This is a common choice but by no means the only possible one.

The word problem for Coxeter groups. A Coxeter matrix is a symmetric matrix $M=\left(m_{s t}\right)_{s, t \in S}$ with $m_{s t} \in\{1,2, \ldots\} \cup\{\infty\}$ and $m_{s t}=1 \Longleftrightarrow s=t$. To a Coxeter matrix $M$ we associate a Coxeter group given by the presentation

$$
W=\left\langle S \mid\left\{(s t)^{m_{s t}} \mid s, t \in S\right\}\right\rangle .
$$


Our main references for Coxeter groups are [8], [19], [36], [47]. See also [7], [25], [33], [48].

Two solutions to the word problem for Coxeter groups were found by Tits. One of them is a consequence of his result that $W$ is isomorphic to a $\operatorname{subgroup}$ of $\operatorname{GL}(n, \overline{\mathbb{Q}})$ (Corollary 1.2.3) where $\overline{\mathbb{Q}}$ denotes an algebraic closure of $\mathbb{Q}$. It is easy to deduce a polynomial (indeed, quadratic) solution to the word problem.

Another solution [46] is elegant because it uses only manipulations of words in $X$. But it is slow: it has an exponential lower bound for time.

In 1993, Brigitte Brink and Bob Howlett, building on ideas of Mike Davis and Mike Shapiro, proved that finite rank Coxeter groups are automatic [10]. Every automatic group has a quadratic solution to the word problem and the involved algorithm is clean and easy to implement [31], [34].

The conjugacy problem for Coxeter groups. In his 1988 thesis [43] supervised by Mike Davis, Gábor Moussong proved that every finite rank Coxeter group acts properly cocompactly on a CAT(0) Euclidean complex. (An action $G \times X \rightarrow X$ is called proper if for every compact set $K \subset X$, the space $\{g \in G \mid K \cap g K \neq \varnothing\}$ is compact.) It is easy to deduce a solution to the conjugacy problem for Coxeter groups. Our version of these ideas can be found in appendix B. Also see the 2007 monograph [25] by Mike Davis.

This solution to the conjugacy problem was the only one so far, and it has both upper and lower time bounds which are exponential.

One of our main aims is to give a polynomial solution (Theorem 6.7.4). It has a lot in common with Tits's first solution to the word problem: it makes extensive use of Tits' faithful representation of Coxeter groups; it is polynomial in time; and it involves algorithms in finite field extensions of $\mathbb{Q}$, making it more wieldy to implement than one might have hoped.

The bulk of the paper doesn't mention words like algorithm and complexity though and is devoted to proving theorems as usual.

One of our main results is Theorem 6.5.14 which states the following. Let $W \rightarrow$ $\mathrm{GL}\left(E^{*}\right)$ be the Tits representation of a Coxeter group $W$ (see Section 1 for details). Suppose that $W$ is irreducible and not affine. Let $w \in W$ not be contained in a parabolic subgroup of $W$ other than $W$ (see Section 2). Then there is a 2-dimensional $w$-invariant subspace $E_{w} \subset E^{*}$ meeting $U^{0}$, the interior of the Tits cone $U$.

The algebraic rank of a group $G$ is the supremum of those $n$ for which $\mathbb{Z}^{n}$ can be embedded in $G$. Another main result is Theorem 6.8.3. It implies that one can read off the algebraic rank of a Coxeter group from the Coxeter matrix. It explicitly gives the commensurability classes of abelian subgroups (two subgroups of $W$ are called commensurable if their intersection has finite index in either).

What we don't do. Our result leaves the following three questions open. 
(a) We prove that every fixed Coxeter group has a polynomial word problem. In other words, the bounds depend on the group. What if the Coxeter group is allowed to vary?

(b) Find an algorithm which is easier to implement than ours.

Is there a property that groups may or may not have (call it property P) such that for every group with property $\mathrm{P}$ there exists a polynomial solution to the conjugacy problem which is easy to implement? If so, can you prove that Coxeter groups have property P?

As to the word problem, one may choose property $\mathrm{P}$ to be automaticity. Coxeter groups are known to be automatic [10].

(c) One may define the input length of a solution to a Dehn problem differently, resulting in a non-equivalent notion of complexity. Instead of the word metric with respect to a finite set $X$ of generators, we suggest the straight line length with respect to $X$ : the straight line length of an element $g \in F(X)$ is the least $k>0$ for which there exist $a_{1}, \ldots, a_{k} \in F(X)$ such that $a_{k}=g$, and for all $p \in\{1, \ldots, k\}$, either $a_{p} \in X$ or there are $i, j<p$ such that $a_{p}=a_{i} a_{j}$ or $a_{p}=a_{i}^{-1}$.

Comparison with the thesis. This article is essentially my 1994 thesis. For ease of reference, I have kept the numbering of the original thesis as far as possible, resulting in some empty sections. The main differences with my thesis are as follows.

I rewrote the introduction. We removed Sections 4.4, 6.6 and 6.9-6.11. Appendix A has been replaced with a mere summary on CAT( 0 ) Euclidean complexes, referring to the literature for all the proofs. We slightly changed the statement of 6.8.2 and the proof of 6.8.3 to fix some unclarity in the proof of 6.8.3.

Many thanks to Bernhard Mühlherr who suggested to include 6.1.2. This also cancels the original item by that number, and simplifies the proof of 6.1.3.

Acknowledgement. As noted above, this paper is essentially my $1994 \mathrm{PhD}$ thesis supervised by Arjeh Cohen at Utrecht. The paper owes an enormous lot to his insights. I am deeply indebted to Arjeh for his careful guidance. It was a wonderful time working with him.

Detailed overview by section. Section 1 collects the preliminaries with references to the literature for their proofs.

For the purpose of this introduction, we mention the following.

A central notion is a root basis, defined in 1.2.1. Every root basis gives rise to a faithful representation of an associated (finite rank) Coxeter group $W$ in a finite dimensional real vector space $E^{*}$.

The Tits cone is a certain convex cone $U \subset E^{*}$ left invariant by $W$. The $W$-action on the interior $U^{0}$ is proper. 
A reflection in $W$ is a conjugate to an element of $S$. Every reflection in $W$ gives rise to a hyperplane: the set of fixed points in $U$. It has codimension 1 .

The set of such hyperplanes is locally finite in $U^{0}$. A chamber is a connected component of $U$ minus all hyperplanes. The Tits cone is the union of the closed chambers.

Section 2. Here we prove some results on the Tits cone which have been known but deserve to be looked at again.

A root basis $(E,(\cdot, \cdot), \Pi)$ is said to be non-degenerate if $(\cdot, \cdot)$ is. We prove the (crucial) results of Section 6.3 under the assumption that the root basis is nondegenerate. Luckily, as we prove in Section 6.1, for every irreducible non-affine Coxeter group there exists a non-degenerate root basis. But it may not be a classical root basis, that is, a root basis where $\Pi$ is a basis for $E$. In Section 2 we pay special attention to non-classical root bases.

For a subset $X \subset U$ we define $X^{\prime} \subset W$ to be the pointwise stabilizer in $W$ of $X$. Conversely, for a subset $H \subset W$ we define $H^{\prime} \subset U$ to be the set of points fixed by each element of $H$. Sets of the form $X^{\prime}$ or $H^{\prime}$ are called stable. The prime maps set up a bijection between stable subsets of $U$ and stable subsets of $W$.

In the case of a classical root basis, the stable subsets of $W$ are precisely the parabolic subgroups of $W$. It follows that every subset $X \subset W$ is contained in a unique smallest parabolic subgroup: its parabolic closure $\operatorname{Pc}(X)$. The parabolic closure $\operatorname{Pc}(w)$ of a single element $w \in W$ is at the center of our attention in later sections.

For non-classical root bases, a parabolic subgroup may not be stable; see 2.1.4 for the correct statement in this direction.

Section 3. We recall in 3.1.3 an important theorem by Deodhar [27], earlier proved by Howlett [35] for finite Coxeter groups. Consider the groupoid (= category whose morphisms are invertible) whose objects are the subsets of $S$ and such that the set of morphisms from $I$ to $J$ is the set of those $g \in W$ for which $g W_{I} g^{-1}=W_{J}$. The result gives an explicit set of generators of this groupoid. In particular, it tells us whether $W_{I}, W_{J}$ are conjugate in $W$.

Using this result, after a little work we arrive at Corollary 3.1 .13 which states the following. The conjugacy problem for Coxeter groups can be solved as soon as for all Coxeter groups, the following two problems can.

(1) Given $w \in W$, determine $I \subset S, g \in W$ such that $\operatorname{Pc}(w)=g W_{I} g^{-1}$.

(2) Given $v, w \in W$ such that $\operatorname{Pc}(v)=\operatorname{Pc}(w)=W$, decide whether $v, w$ are conjugate in $W$.

A polynomial algorithm for (1) is obtained later on in 5.10.9 and for (2) in 6.4.4 as well as in 6.7.4.

In Section 3.2 we study finite parabolic subgroups. In particular, we solve both (1) and (2) if $v, w$ are torsion (3.2.1 (b) and 3.2.2). 
Section 4. By a hyperbolic reflection group we mean a discrete subgroup of the isometry group of hyperbolic space, generated by finitely many reflections. Such a group is always a Coxeter group.

This should not be confused with word hyperbolic groups. See Section 4.3 for a definition of this and a comparison of the two concepts.

Suppose that $(E,(\cdot, \cdot), \Pi)$ is a root base for $W$ such that the symmetric bilinear form $(\cdot, \cdot)$ has signature $(n, 1)$. Then $W$ is a hyperbolic reflection group; indeed hyperbolic space $\mathbb{H}^{n}$ is one of the two components of $\left\{\mathbb{R}_{>0} x \mid x \in E,(x, x)<0\right\}$.

It is known that if $g$ is an isometry of hyperbolic space $\mathbb{H}^{n}$ then precisely one of the following is true (see 4.5.1).

(a) $g$ has a fixed point in $\mathbb{H}^{n}$.

(b) $g$ has no fixed points in $\mathbb{H}^{n}$, and has exactly one fixed point at infinity.

(c) $g$ has no fixed points in $\mathbb{H}^{n}$, and has exactly two fixed points at infinity.

In the above cases we call $g$, respectively, elliptic, parabolic, and hyperbolic.

We find this trichotomy a useful guidance. In Section 6 we will find a rather similar trichotomy for Coxeter groups.

The main result of Section 4 is 4.7.3 stating that if $w \in W$ is parabolic in the above sense and fixes $\mathbb{R}_{>0} x \in \overline{\mathbb{H}}^{n}$, then $x \in U$. In Theorem 4.7.6 we use this to give a solution to the conjugacy problem for hyperbolic reflection groups.

Section 5. For a root $\alpha$, let $f_{\alpha}: U^{0} \rightarrow\{-1 / 2,0,1 / 2\}$ be the map

$$
f_{\alpha}(x)= \begin{cases}-1 / 2 & \text { if }\langle x, \alpha\rangle<0 \\ 0 & \text { if }\langle x, \alpha\rangle=0 \\ 1 / 2 & \text { if }\langle x, \alpha\rangle>0\end{cases}
$$

We define pseudometrics $d_{\alpha}$ and $d_{\Phi}$ on $U^{0}$ by

$$
d_{\alpha}(x, y)=\left|f_{\alpha}(x)-f_{\alpha}(y)\right|, \quad d_{\Phi}(x, y)=\sum_{\alpha \in \Phi^{+}} d_{\alpha}(x, y) .
$$

Then $d_{\Phi}(x, y)=0$ if and only if $\{x, y\}$ is contained in some facet. Moreover, $d_{\Phi}$ generalizes the metric $d_{W}$ in the sense that $d_{W}(x, y)=d_{\Phi}(x C, y C)$ for all $x, y \in W$.

In this section we fix an element $w \in W$. We define the axis $Q(w)$ by

$$
\left\{x \in U^{0} \mid \text { for all } n \in \mathbb{Z}: d_{\Phi}\left(x, w^{n} x\right)=|n| d_{\Phi}(x, w x)\right\} .
$$

Examples of pictures of $Q(w)$ can be found in Figures 5-7. We prove that $Q(w)$ is non-empty in 5.6.10.

A large part of Section 5 is devoted to distinguishing different sorts of roots with respect to $w$. For example, a root $\alpha$ is said to be $w$-periodic if there exists $n>0$ such that $w^{n} \alpha=\alpha$. 
We often say periodic instead of $w$-periodic if there is no danger of confusion, and likewise for the other classes of roots.

There is a bijection between the set $\Phi$ of roots and the set of half-spaces. If the root $\alpha$ corresponds to the half-space $A$ then we say that $A$ satisfies some property (such as being periodic) if $\alpha$ does. Also, we have a 2-to-1 map from $\Phi$ to the set of reflections in $W$. If $\alpha$ has a property then we say that the corresponding reflection $r_{\alpha}$ has, provided this doesn't lead to any confusion.

In Figure 1 we give an overview of the classes of roots and their definitions.

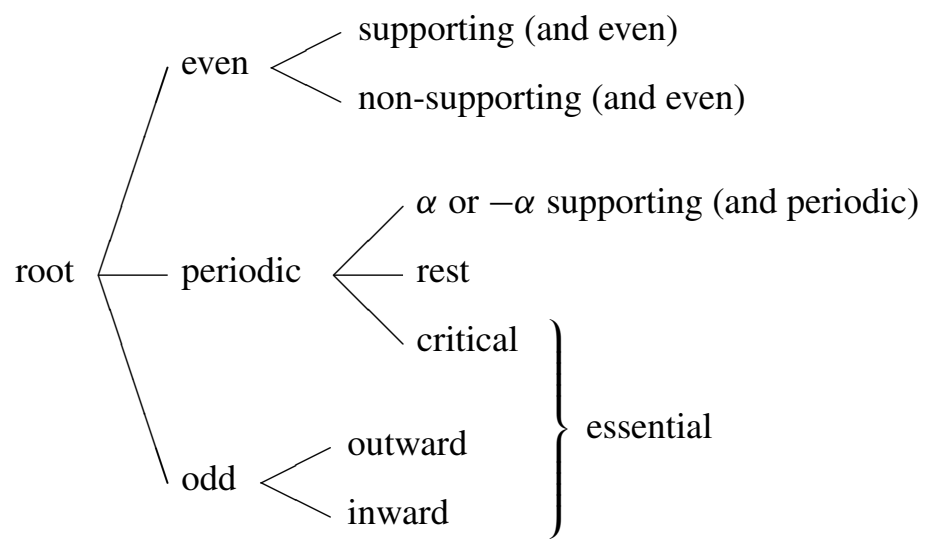

Let $\alpha$ be a root and let $A$ denote the corresponding half-space.

We say that $A$ is periodic if there exists $n>0$ such that $w^{n} A=A$.

We say that $A$ is even if it is not periodic and for all $x \in W$, the number $\#\{n \in \mathbb{Z} \mid$ $A$ separates $w^{n} x$ from $\left.w^{n+1} x\right\}$ is finite and even.

We say that $A$ is odd if it is not periodic and for all $x \in W$, the number $\#\{n \in \mathbb{Z} \mid$ $A$ separates $w^{n} x$ from $w^{n+1} x$ \} is finite and odd.

We say that $A$ is supporting if $Q(w) \subset H(\alpha):=\left\{x \in U^{0} \mid\langle x, \alpha\rangle>0\right\}$.

We say that $A$ is critical if it satisfies the following equivalent conditions.

- Span $w^{\mathbb{Z}} \alpha$ is positive definite and $\sum_{n=1}^{k} w^{n} \alpha=0$, where $k$ is the smallest positive integer with $w^{k} \alpha=\alpha$.

- $\bigcap_{n \in \mathbb{Z}} w^{n} A=\bigcap_{n \in \mathbb{Z}} w^{n}(W-A)=\varnothing$, and $A$ is periodic.

- $Q(w) \subset \mu(\alpha)$.

As the diagram suggests, a periodic root $\alpha$ is called rest if it is not critical and neither $\alpha$ nor $-\alpha$ are supporting.

An odd root $\alpha$ is said to be outward if for some (hence all) $x \in U^{0}$ the following holds. For almost all $n \in \mathbb{Z}$, we have $n\left\langle w^{n} x, \alpha\right\rangle<0$. It is called inward if $-\alpha$ is outward.

A root is called essential if it is odd or critical.

Figure 1. Classes of roots and their definitions. 
In Theorem 5.8.3 we prove that the parabolic closure $\operatorname{Pc}(w)$ of $w$ is the subgroup of $W$ generated by the $w$-essential reflections. Also, $\operatorname{Pc}(w)$ is a direct product $\mathrm{Pc}^{\infty}(w) \times \mathrm{Pc}^{0}(w)$ where $\mathrm{Pc}^{\infty}(w)$ is generated by the odd reflections and $\mathrm{Pc}^{0}(w)$ by those reflections $r_{\alpha}$ for which $\alpha$ is perpendicular to all odd roots.

In Section 5.10 we prove that there are polynomial algorithms doing all sorts of related computations. For example, on input $w, \alpha$ one algorithm (5.10.1) decides to which of the classes the root $\alpha$ belongs. Another example is an algorithm (5.10.9) that, on input $w$, finds $I \subset S$ and $g \in W$ such that $\operatorname{Pc}(w)=g W_{I} g^{-1}$. Most of the algorithms make extensive use of the Tits cone.

Section 6. In 3.1.13 and 5.10.9, the conjugacy problem for Coxeter groups has been reduced to the case $\operatorname{Pc}(v)=\operatorname{Pc}(w)=W$. In Section 6, we solve the conjugacy problem for this case.

We treat affine and non-affine groups separately. The affine groups have been taken care of in 4.2.1.

In Section 6.1 it is shown that for irreducible infinite non-affine Coxeter groups, there exists a non-degenerate root basis, which means that the radical $E^{\perp}=\{x \in$ $E \mid(x, y)=0$ for all $y \in E\}$ is zero. Indeed, every root basis $E$ gives rise to one, obtained by dividing out the radical.

In Section 6.2 we prove some useful inequalities, such as Corollary 6.2.3 which states that if $\alpha<\beta<\gamma$ are roots then $(\alpha, \beta) \leq(\alpha, \gamma)$ and $(\beta, \gamma) \leq(\alpha, \gamma)$.

From here on, we assume $(W, S)$ to be irreducible, infinite and non-affine, unless stated otherwise. Furthermore, an element $w \in W$ with $\operatorname{Pc}(w)=W$ is fixed. By 5.8.7, we have $\operatorname{Pc}^{\infty}(w)=W$, and $\operatorname{Span}(\Pi)$ is spanned by the outward roots.

By 6.1.3 there exists a non-degenerate root basis $(E,(\cdot, \cdot), \Pi)$ for $W$. Also we may assume that $E$ is spanned by $\Pi$ and hence by the odd roots. Such $E$ is fixed from now on.

A key section is 6.3. One useful result from this section is Corollary 6.3.8 stating that for all outward $\alpha, \beta$ one has $\left(\alpha, w^{n} \beta\right) \rightarrow \infty$ as $n \rightarrow \infty$ or $n \rightarrow-\infty$. Another is Corollary 6.3.11 which says that for all outward $\alpha, \beta$ one has $w^{-n} \alpha<\beta<w^{n} \alpha$ for almost all $n>0$. A result of independent interest is Corollary 6.3.10 stating that $w^{\mathbb{Z}}$ has finite index in the centralizer of $w$.

In Section 6.4 we prove that every Coxeter group of rank $r$ has a polynomial time conjugacy problem with exponent $r+3$ (Theorem 6.4.4). This is not our fastest algorithm, which will be obtained in a later section. Another result, unrelated to polynomial algorithms, is Corollary 6.4.7 stating that for every finite rank Coxeter group $W$ there exists a constant $N=N(W)$ such that if $v, w \in W$ are conjugate, then there exists $g \in W$ with $g v g^{-1}=w$ and $\ell(g) \leq N(\ell(v)+\ell(w))$.

We turn to Section 6.5. Let $\lambda$ denote the maximum of the absolute values of the complex eigenvalues of $w$. Let $E_{\mu} \subset E^{*}$ denote the generalized eigenspace of eigenvalue $\mu$, for all $\mu$. 
A relatively easy lemma (6.5.4) states that $\lambda>1$, that $\lambda$ is an eigenvalue of $w$, and that if $\mu$ is any eigenvalue of $w$ such that $|\mu|=\lambda$, then $(w-\mu) E_{\mu}=0$.

The larger part of Section 6.5 is devoted to proving that $\lambda$ is a simple eigenvalue. This is proved in Theorem 6.5.14, which also yields that the set $Q_{0}(w):=\left(E_{\lambda} \oplus\right.$ $\left.E_{\lambda-1}\right) \cap U^{0}$ is 2-dimensional, indeed, of the form $\mathbb{R}_{>0} u_{1} \oplus \mathbb{R}_{>0} u_{2}$ for independent $u_{1}, u_{2} \in E^{*}$. It is the closest possible analogue to the axis in real hyperbolic space of a hyperbolic element. It is clear that $Q_{0}(w) \subset Q(w)$.

Theorem 6.7.4 states that for every (finite rank) Coxeter group there exists a cubic solution to the conjugacy problem, that is, a polynomial one with exponent 3 . The proof relies on our knowledge of $Q_{0}(w)$.

Section 6.8 presents some results of independent interest. They are not related to algorithms, and are relatively easily proved using some of our results.

Let $I_{1}, \ldots, I_{n} \subset S$ be irreducible, non-spherical and pairwise perpendicular. For all $i$, let $H_{i} \subset W_{I_{i}}$ be a subgroup as follows. If $I_{i}$ is affine, $H_{i}$ is the translation subgroup. Otherwise, $H_{i}$ is any subgroup of $W_{I_{i}}$ isomorphic to $\mathbb{Z}$. We call $\prod_{i} H_{i}$ a standard free abelian subgroup.

The main result of this section is Theorem 6.8.3 which states the following. Each free abelian subgroup of a finite rank Coxeter group $W$ has a finite index subgroup which is conjugate to a subgroup of a standard free abelian subgroup.

Appendix A. In this appendix we collect all definitions and results one needs to know to be able to read Appendix B, and we point to the proofs in the literature. Our main reference is [9].

The appendix begins with a short introduction to metric spaces and their geodesics in Section A.1. A geodesic space is a metric space in which any two points can be connected by a geodesic.

A Euclidean cell is the convex hull of finitely many points in Euclidean space $\mathbb{E}^{n}$ with the usual metric. A Euclidean complex is what you get if you glue a bunch of Euclidean cells together by isometries of faces. By a metric complex we mean a Euclidean complex or its cousin, a spherical complex. In Section A. 2 we look at metric complexes.

A geodesic space $X$ is called CAT(0) if, roughly, triangles in $X$ are no fatter than in the Euclidean plane. For example, a simply connected complete Riemannian manifold is CAT(0) if and only if it has nonpositive curvature.

Just as in the tangent space to a point of a Riemannian manifolds there is a unit sphere, every point in a spherical complex has a tangent-space-like-thing with a spherical complex in it. The latter is called the link, and it is a spherical complex. One can repeat the link operation to obtain more complexes of ever smaller dimensions; they are also called links.

As we are interested in constructing an action of a Coxeter group on a CAT(0) Euclidean complex, we need a way to prove that a Euclidean complex is CAT(0) if it is. This is provided by Theorem A.3.2. The condition is that every link satisfies the 
girth condition, that is, its minimal cycles have length at least $2 \pi$.

Appendix B. In his 1988 thesis [43] supervised by Michael Davis, Gábor Moussong proved that every finite rank Coxeter group acts properly cocompactly on a CAT(0) Euclidean complex. We call it the Davis-Moussong complex M. Appendix B presents our approach to it.

The appendix begins with Section B. 1 on certain spherical complexes called nerves of almost negative matrices, or just nerves. Nerves satisfy the girth condition, see B.1.1. Moreover, links of nerves are themselves nerves (B.1.2).

One difference between our approach and Moussong's is that we build $M$ from one (Euclidean) cell for each finite parabolic subgroup, whereas Moussong builds his complex from many copies of one thing, one for each element of $W$. The Euclidean cells involved in our construction are studied in Section B.2.

In Section B.3 we construct the Davis-Moussong complex. Every link in $M$ is a nerve so $M$ is CAT(0) (see B.3.3).

In Section B.4 we construct an embedding $f: M \rightarrow U^{0}$ of the Davis-Moussong complex in the interior of the Tits cone. For every cell $C \in M$, the bilinear form $(\cdot, \cdot)$ on $\operatorname{Ann}(C) \subset E$ is positive definite. It follows that $\operatorname{Span}(C)$ has a Euclidean metric. The metric on $C$ inherited from $\operatorname{Span}(C)$ is the metric in $M$. This yields a different but equivalent construction of the Davis-Moussong complex.

One of the reasons we are interested in the Davis-Moussong complex is that it gives rise to a (slow) algorithm to the conjugacy problem for Coxeter groups. We prove this in Proposition B.6.1 after some preparation in Section B.5.

\section{Coxeter groups}

1.1. Coxeter groups. A Coxeter matrix is a symmetric matrix $M=\left(m_{i j}\right)_{i, j \in X}$ indexed by a set $X$, with entries in $\{1,2, \ldots\} \cup\{\infty\}$ such that $m_{i j}=1 \Longleftrightarrow i=j$. The Coxeter group associated to $M$ is the group generated by elements $s_{i}(i \in X)$ subject to the relations

$$
\left(s_{i} s_{j}\right)^{m_{i j}}=1 \text {. }
$$

If $m_{i j}=\infty$, there is no relation. The Coxeter group is denoted by $W(M)$ or just $W$. Write $S=\left\{s_{i} \mid i \in X\right\} \subset W$. A Coxeter system is a pair $\left(W^{\prime}, S^{\prime}\right)$ of a group $W^{\prime}$ and a subset $S^{\prime} \subset W^{\prime}$ such that there exists a Coxeter matrix $M$ and a group isomorphism $W(M) \rightarrow W^{\prime}$, mapping $S$ to $S^{\prime}$. The cardinality of $X$ is called the rank of the system (or group).

Let $(W, S)$ be a Coxeter system. Following [8], we construct a representation of $W$ as follows. Let $E$ be a real vector space with basis $\left\{e_{i} \mid i \in X\right\}$. Define a symmetric bilinear form $(\cdot, \cdot)$ on $E$ by $\left(e_{i}, e_{j}\right)=-\cos \left(\pi / m_{i j}\right)$. We let $s_{i}$ act on $E$ as the orthogonal reflection in $e_{i}$, that is, $s_{i} x=x-2\left(e_{i}, x\right) e_{i}$ for all $x \in E$. It is easy to check that this extends to a representation $W \rightarrow \mathrm{GL}(E)$. 
In particular, the map $i \mapsto s_{i}$ is bijective. We prefer $S$ over $X$ as the indexing set, that is, we write $m_{s t}=m_{i j}$ where $s=s_{i}, t=s_{j}$, and $e_{s}=e_{i}$ where $s=s_{i}$. Another consequence of the representation is that $m_{s t}$ is the exact order of $s t$. Hence, the Coxeter matrix is determined by the Coxeter system.

For $w \in W$, define the length $\ell(w)=\min \left\{k \mid w=s_{1} \ldots s_{k}\right.$ for some $\left.s_{1}, \ldots, s_{k} \in S\right\}$. We define a metric $d_{W}$ on $W$ by $d_{W}(x, y)=\ell\left(x^{-1} y\right)$.

Thus, the metric space $\left(W, d_{W}\right)$ is acted upon by $W$ from the left by multiplication. In the sequel, we will define two more metric spaces, acted upon by $W$. The actions will always be from the left.

The Cayley graph of $(W, S)$ is the graph with vertex set $W$ and edges $\{x, x s\}$, $x \in W, s \in S$. The edge $\{x, x s\}$ is sometimes labelled $s$. The metric $d_{W}$ is nothing but the path metric of the Cayley graph.

The Coxeter graph is the graph with vertex set $S$ and labelled edges $\left\{\{s, t\} \mid m_{s t}>\right.$ 2 ) with labels $m_{s t}$. Usually, labels 3 are omitted. The Coxeter system (or group) is called irreducible if the Coxeter graph is connected.

For a subset $I \subset S$, we denote the subgroup of $W$ generated by $I$ by $W_{I}$. It is called a standard parabolic subgroup of $W$. Note that a subgroup of $W$ is a standard parabolic subgroup if and only if it is connected in the Cayley graph. A parabolic subgroup is a subgroup of $W$ conjugate to a standard parabolic one.

\subsection{Root bases}

1.2.1 Definition. A root basis is a triple $(E,(\cdot, \cdot), \Pi)$ of a real vector space $E$, a symmetric bilinear form $(\cdot, \cdot)$ on $E$ and a finite set $\Pi \subset E$ such that:

(1) For all $\alpha \in \Pi:(\alpha, \alpha)=1$.

(2) For all different $\alpha, \beta \in \Pi:(\alpha, \beta) \in\left\{-\cos (\pi / m) \mid m \in \mathbb{Z}_{>1}\right\} \cup(-\infty,-1]$.

(3) There exists $x \in E^{*}$ such that $\langle x, \alpha\rangle>0$ for all $\alpha \in \Pi$.

Here $E^{*}$ denotes the dual of $E$, and $\langle\cdot, \cdot\rangle: E^{*} \times E \rightarrow \mathbb{R}$ is the pairing.

We are grateful to Bernhard Mühlherr for pointing out that (3) is often a consequence of (1) and (2). See 6.1.2 for the precise statement and a proof.

Probably many of our results on root bases do not require the assumption that $\Pi$ is finite. We will not go into this.

Note that $\Pi$ does not have to span $E$, nor be independent. If $(E,(\cdot, \cdot), \Pi)$ is a root basis, then so is $\left(\operatorname{Span} \Pi,(\cdot, \cdot)^{\prime}, \Pi\right)$, where $(\cdot, \cdot)^{\prime}$ is the restriction of $(\cdot, \cdot)$ to Span $\Pi$. An example of a root basis where $\Pi$ is dependent is associated to a quadrangle in the hyperbolic plane with angles $\pi / m_{s t}$ (see Section 4.6). Another example will be given in 6.1.4.

Given a Coxeter matrix $\left(m_{s t}\right)_{s, t \in S}$, a natural root basis is the one briefly mentioned in the previous section, and which is defined by $E=\bigoplus_{s \in S} \mathbb{R} e_{s},\left(e_{s}, e_{t}\right)=$ $-\cos \left(\pi / m_{s t}\right)\left(=-1\right.$ if $\left.m_{s t}=\infty\right), \Pi=\left\{e_{s} \mid s \in S\right\}$. Note that (3) is satisfied by 
$x \in E^{*}$ defined by $\left\langle x, e_{s}\right\rangle=1$ for all $s \in S$. We will sometimes refer to this as the classical root basis.

Fix a root basis $(E,(\cdot, \cdot), \Pi)$. For $\alpha \in \Pi$, define $r_{\alpha} \in \operatorname{GL}(E)$ by $r_{\alpha}(x)=$ $x-2(x, \alpha) \alpha$. We define $S=\left\{r_{\alpha} \mid \alpha \in \Pi\right\}, W=\langle S\rangle \subset \operatorname{GL}(E)$. The dual action of $W \subset \mathrm{GL}(E)$ on $E^{*}$ is defined by $\langle w x, w y\rangle=\langle x, y\rangle$. Write $e_{s}=\alpha$ whenever $\alpha \in \Pi, s=r_{\alpha}$. For a subset $I \subset S$, write $\Pi_{I}=\left\{e_{i} \mid i \in I\right\}$. We sometimes write $a_{s t}=\left(e_{s}, e_{t}\right)$. For a subset $I \subset S$, define the Gram matrix $A_{I}=\left(a_{s t}\right)_{s, t \in I}$, and write $E_{I}=\operatorname{Span} \Pi_{I}$.

In the case where $\Pi$ is a basis of $E$, it is sometimes convenient to use the basis $\left\{f_{s}\right\}$ of $E^{*}$ dual to $\left\{e_{s}\right\}$, which is defined by $\left\langle f_{s}, e_{t}\right\rangle=\delta_{s t}$.

We define

$$
C=\left\{x \in E^{*} \mid\left\langle x, e_{s}\right\rangle>0 \text { for all } s \in S\right\} .
$$

Observe that $C \neq \varnothing$ by (3). For every $I \subset S$, define the face

$$
C_{I}=\left\{x \in E^{*} \mid\left\langle x, e_{s}\right\rangle=0 \text { for all } s \in I,\left\langle x, e_{s}\right\rangle>0 \text { for all } s \in S-I\right\} .
$$

We call a set $I \subset S$ facial if $C_{I} \neq \varnothing$. Note that $C_{\varnothing}=C$ and $\bar{C}=\bigsqcup_{I \subseteq S} C_{I}$, where a bar denotes topological closure. We define the Tits cone $U=\bigcup\{w \bar{C} \mid w \in W\}$. Its topological interior relative to $E^{*}$ is denoted by $U^{0}$. A facet is a set of the form $w C_{I}, w \in W, I \subset S$. The dimension of a facet is by definition the dimension of its linear span.

For two points $x, y$ in a real vector space, write $[x, y]=\{t x+(1-t) y \mid t \in[0,1]\}$. A subset $X$ of a real vector space is called convex if for all $x, y \in X$, we have $[x, y] \subset X$.

1.2.2 Theorem. Let $(E,(\cdot, \cdot), \Pi)$ be a root basis, and retain the notations of above. Then the following holds.

(a) $(W, S)$ is a Coxeter system, and $W$ is a discrete subgroup of $\operatorname{GL}(E)$.

(b) Let $w \in W, s \in S$. Then either

$$
\left\langle w C, e_{s}\right\rangle \subset \mathbb{R}_{>0} \text { and } \ell(s w)>\ell(w)
$$

or

$$
\left\langle w C, e_{s}\right\rangle \subset \mathbb{R}_{<0} \text { and } \ell(s w)<\ell(w) .
$$

(c) Let $w \in W, I, J \subset S$. If $C_{I} \cap w C_{J} \neq \varnothing$ then $I=J$ and $w \in W_{I}$.

(d) $U$ is convex. If $x, y \in U$ then there are only finitely many facets meeting $[x, y]$.

Proof. For the classical root basis, proofs can be found in [36], 5.13, [8], Theorem 1, Chapter 4.4. For other root bases, see [47] or apply 6.1.3. 
1.2.3 Corollary. (a) For all $x \in C_{I}$, the stabilizer in $W$ of $x$ is $W_{I}$.

(b) Let $I \subset S$. Then $\left(W_{I}, I\right)$ is a Coxeter system, and its length function is the restriction to $W_{I}$ of the length function on $W$.

(c) Let $I, J \subset S$. Then $W_{I} \cap W_{J}=W_{I \cap J}$.

(d) The word problem for Coxeter groups is solvable.

By 1.2.3 (c), if $X \subset W$ is a subset, then there exists a smallest standard parabolic subgroup of $W$ containing $X$. It is called the standard parabolic closure of $X$.

1.2.4 Proposition. There exists an algorithm that, given $x \in U$, finds $w \in W$ such that $w x \in \bar{C}$.

Proof. We describe the algorithm. Let $x_{0} \in U$ be given, and put $w_{0}=1 \in W$. Compute $x_{k}, w_{k}(k=1,2, \ldots)$ which are (non-uniquely) defined as follows. Suppose that $x_{k} \notin \bar{C}$. Choose $s \in S$ such that $\left\langle x_{k}, e_{s}\right\rangle<0$. Let $x_{k+1}=s x_{k}, w_{k+1}=s w_{k}$. If $x_{k} \in \bar{C}$, then the algorithm finishes. We have $x_{k}=w_{k} x_{0}$ for all $k$. We will show that the algorithm terminates. Let $v \in W$ such that $x_{0} \in v \bar{C}$. Choose $y_{0} \in v C$ and write $y_{k}=w_{k} y_{0}$. Then for all $s \in S$, the condition $\left\langle x_{k}, e_{s}\right\rangle<0$ implies $\left\langle y_{k}, e_{s}\right\rangle<0$, which shows that the sequence $y_{k}$ is also a sequence satisfying the conditions of the algorithm. Hence $\ell\left(w_{k}\right) \leq \ell(v)$ whenever $w_{k}$ is defined. By 1.2.2(b), we have $\ell\left(w_{k}\right)=k$, which shows that the algorithm terminates.

A root is an element in $E$ of the form $w e_{s}, w \in W, s \in S$. The set of roots is denoted by $\Phi$ and is called the root system associated to $(W, S)$. To each $\alpha \in \Phi$ we associate a half-space $A(\alpha) \in W$ by

$$
A(\alpha)=\left\{w \in W \mid\langle w C, \alpha\rangle \subset \mathbb{R}_{>0}\right\},
$$

and a reflection $r_{\alpha} \in W$ by

$$
r_{\alpha}(x)=x-2(x, \alpha) \alpha .
$$

Note that $W=A(\alpha) \sqcup A(-\alpha)$ for every $\operatorname{root} \alpha$. Define $\Phi^{+}=\{\alpha \in \Phi \mid 1 \in A(\alpha)\}$, $\Phi^{-}=-\Phi^{+}$, so that $\Phi=\Phi^{+} \sqcup \Phi^{-}$. Elements of $\Phi^{+}$are called positive roots, the others negative. If a root $\alpha=\sum_{s \in S} x_{s} e_{s}$ is positive and $\Pi$ is independent, then $x_{s} \geq 0$ for all $s \in S$.

A standard parabolic root subsystem is a set of the form $\Phi_{I}=\left\{w e_{s} \mid w \in W\right.$, $s \in I\}$, where $I \subset S$. A parabolic root system is a set of the form $w \Phi_{I}, w \in W$, $I \subset S$.

The set of half-spaces is in bijection with $\Phi$ by $\alpha \mapsto A(\alpha)$. We sometimes write $r_{A}=r_{\alpha}$ if $A=A(\alpha)$. By 1.2.2(b), half-spaces can be characterized completely combinatorially - for example, $A\left(e_{s}\right)=\{w \in W \mid \ell(s w)>\ell(w)\}$.

Let $\alpha \in \Phi, A=A(\alpha), x, y \in W$. We say that $\alpha$ (or $A$, or $r_{\alpha}$ ) separates $x$ from $y$ if $A$ contains precisely one of $x, y$. The distance $d_{W}(x, y)$ equals the number of reflections separating $x$ from $y$. 
1.2.5 Lemma. $\Phi$ is discrete in $E$.

Proof. Consider the map $R:\{\alpha \in E \mid(\alpha, \alpha)=1\} \rightarrow \operatorname{GL}(E), \alpha \mapsto r_{\alpha}=(x \mapsto$ $x-2(x, \alpha) \alpha)$. Since $R$ is continuous and $W \subset \operatorname{GL}(E)$ is discrete, $R^{-1}(W)$ is discrete in $E$ and hence so is $\Phi \subset R^{-1}(W)$.

1.2.6 Proposition. Let $(W, S)$ be a Coxeter system and let $E$ be the classical root basis. The following conditions are equivalent.

(a) $W$ is finite.

(b) $\Phi$ is finite.

(c) $U=E^{*}$.

(d) $C \cap-U \neq \varnothing$.

(e) The bilinear form $(\cdot, \cdot)$ on $E$ is positive definite.

(f) There exists $w \in W$ such that for all $s \in S: \ell(w s)<\ell(w)$.

(g) There exists $w \in W$ such that for all $v \in W-\{w\}: \ell(v)<\ell(w)$.

(h) There exists $w \in W$ such that $w \Pi=-\Pi$.

Moreover, if these conditions hold, then the elements in (f), (g) and (h) are unique and equal.

Proof. A hint to a proof is given in [8], Chapter 5, p. 130, Exercise 2.

The irreducible finite Coxeter groups have been classified by Coxeter [23].

We call $I \subset S$ spherical if $W_{I}$ is finite. For every spherical $I \subset S$, there is a unique longest element in $W_{I}$ by 1.2 .6 , which is denoted by $w_{I}$.

In this article, unless stated otherwise, we fix a finite rank Coxeter system $(W, S)$ and a root basis $(E,(\cdot, \cdot), \Pi)$ associated to it.

1.3. Convexity and gatedness. The results of this section will be used in $2.2 .5,3.1 .8$ and B.4.1.

1.3.1 Proposition. Let $X \subset W$ be a set. The following are equivalent.

(1) Let $x, z \in X, y \in W$ with $d_{W}(x, z)=d_{W}(x, y)+d_{W}(y, z)$. Then $y \in X$.

(2) $X$ is the intersection of a family of half-spaces.

Proof. (1) $\Longrightarrow$ (2). Let $p \in W-X$. Choose $x \in X$ closest to $p$. Let $s \in S$ be such that $y=x s$ is closer to $p$ than $x$ is. Let $A$ be the half-space containing $x$ but not $y$. We have $p \notin A$. The proof will be finished once we have shown $X \subset A$. Let $z \in X-A$. Note that $d(x, z)=d(x, y)+d(y, z)$. By (1), $y \in X$, a contradiction. Hence $X \subset A$, which concludes the proof. (2) $\Longrightarrow(1)$ is trivial. 
1.3.2 Definition. A set $X \subset W$ is called convex if it satisfies the properties of 1.3.1. Some authors call it geodesically closed. We call $X$ gated if for every $x \in W$, there exists $y \in X$ such that for every half-space $A$ with $y \in A, x \notin A$, we have $X \subset A$.

1.3.3 Proposition. (a) A set $X \subset W$ is gated if and only if for every $x \in W$, there exists $y \in X$ such that for all $z \in X$, we have $d_{W}(x, z)=d_{W}(x, y)+d_{W}(y, z)$. The points $y$ of the two definitions are equal and unique.

(b) Gatedness implies convexity.

Proof. Left to the reader.

1.3.4 Definition. Let $I, J \subset S$. We define $D_{I J}$ to be the set of elements in $W$ such that $\ell(s w)>\ell(w)$ for all $s \in I$ and $\ell(w s)>\ell(w)$ for all $s \in J$.

1.3.5 Proposition. (a) Let $I, J \subset S, w \in W$. Then the set $W_{I} w W_{J} \cap D_{I J}$ consists of precisely one element. Every element of $w \in W$ has a (not necessarily unique) expression $w=u d v$, with $u \in W_{I}, d \in D_{I J}, v \in W_{J}, \ell(w)=\ell(u)+\ell(d)+\ell(v)$.

(b) Let $I \subset S$. The map $W_{I} \times D_{I \varnothing} \rightarrow W,(x, y) \mapsto x y$ is a bijection. For all $x \in W_{I}, y \in D_{I \varnothing,}$ we have $\ell(x y)=\ell(x)+\ell(y)$.

(c) $W_{I}$ is gated.

(d) If $w \in D_{I J}$ then $W_{I} \cap w W_{J} w^{-1}=\left\langle I \cap w J w^{-1}\right\rangle$.

Proof. For (a) and (b), see [8], Exercise 1.3, Chapter 4. Part (c) is easily seen to be equivalent to (b). For (d), see [45], Lemma 2.

1.3.6 Corollary. Let $w=s_{1} \ldots s_{n}, s_{i} \in S, \ell(w)=n$. Then the standard parabolic closure of $w$ equals $W_{I}$, with $I=\left\{s_{1}, \ldots, s_{n}\right\}$.

Proof. [19], 5.1 (ii).

1.3.7 Corollary. Let $w \in W, I=\{s \in S \mid \ell(s w)<\ell(w)\}$. Then $I$ is spherical and $\ell(w)=\ell\left(w_{I} w\right)+\ell\left(w_{I}\right)$.

Proof. By 1.3.5(b), there exist (unique) $x \in W_{I}, y \in D_{I \varnothing}$ such that $w=x y$, $\ell(w)=\ell(x)+\ell(y)$. For all $s \in I$, we have $\ell(s x)<\ell(x)$ because, again using 1.3.5(b),

$$
\ell(s x)+\ell(y)=\ell(s x y)=\ell(s w)<\ell(w)=\ell(x)+\ell(y) .
$$

By 1.2.6, $I$ is spherical, and $x=w_{I}$. This proves the corollary. 
1.4. Reflection subgroups. We will give two basic theorems $(1.4 .2,1.4 .3)$ on reflection subgroups, essentially shown by Deodhar [28] and independently Dyer [30]. We study in more detail subgroups generated by two roots in 1.4.7. This provides a link between inner products of roots and the combinatorics of Coxeter groups, which will be used, among others, in 5.3.2, 5.6.5, 6.2.2 and 6.5.6.

1.4.1 Theorem. Let $W$ be a group generated by a set $S$ of involutions, and suppose that $W$ acts on a set $W^{\prime}$. Let there be given $p \in W^{\prime}$, and for each $s \in S$ a set $A_{s} \subset W^{\prime}$ such that the following holds.

(1) $\{p\}=\bigcap_{s \in S} A_{s}$.

(2) $W^{\prime}=A_{s} \sqcup s A_{s}$ for all $s \in S$.

(3) If $w \in\langle s, t\rangle, s, t \in S$, then $w\left(A_{s} \cap A_{t}\right)$ is contained in either $A_{s}$ or $s A_{s}$, and in the latter case, we have $\ell(s w)<\ell(w)$.

Then $(W, S)$ is a Coxeter system and the $W$-action on $W^{\prime}$ is simply transitive.

Proof. The proof is essentially the same as that of [8], Theorem 1, p. 93.

Let $(W, S)$ be a Coxeter system. To an edge $\{x, x s\}$ in the Cayley graph of $(W, S)$, we associate a reflection $x s x^{-1}$. It is the (unique) reflection reversing the edge. Any reflection is associated to at least one edge.

1.4.2 Theorem. Let $X \subset W$ be a set of reflections, such that for all $x, y \in X$, we have $x y x \in X$. Let $H=\langle X\rangle$. Let $\Gamma$ be the Cayley graph of $W$ minus those edges reversed by an element of $X$. Let $H^{\prime}$ be the set of connected components of $\Gamma$. Let $p \in H^{\prime}$ be the component containing 1 . Let $T$ be the set of reflections reversing an edge from $p$ to $W-p$. Then the following holds.

(a) $(H, T)$ is a Coxeter system.

(b) $H$ acts simply transitively on $H^{\prime}$.

(c) $X$ equals the set of reflections in $H$.

Proof. For each $t \in T$, define $A_{t} \subset H^{\prime}$ by $A_{t}=\{w p \mid w \in H, t$ separates 1 from $w$ \}. We leave it to the reader to check that conditions (1), (2), (3) of 1.4 .1 are verified. Application of 1.4.1 immediately shows (a) and (b). In order to prove (c), let $r \in H$ be a reflection. Let $\{x, x s\}$ be an edge reversed by $r$. Hence $x$ and $x s$ are in different components of $\Gamma$ by (b) and since $r \neq 1$. This shows that $r \in X$.

In the above theorem, $T$ is called the canonical set of generators of $H$.

By a reflection subgroup of $W$ we mean a subgroup generated by reflections. A root subsystem of $\Phi$ is a subset $X \subset \Phi$ such that for all $\alpha, \beta \in X$, we have $r_{\alpha} \beta \in X$. By 1.4.2(c), there is a bijection between reflection subgroups $H \subset W$ and root subsystems of $\Phi$, defined by $H \mapsto\left\{\alpha \in \Phi \mid r_{\alpha} \in H\right\}$. 
Let $X \subset \Phi$ be a root subsystem and let $H$ be the corresponding subgroup. Let $Y \subset X$ denote a minimal non-empty subset such that $Y^{\perp} \cap X=X-Y$. Then $Y$ is called a component of $X$ and $\left\langle\left\{r_{\alpha} \mid \alpha \in Y\right\}\right\rangle$ is a component of $H$. For subsets $I \subset S$, a component of $I$ is by definition a component in the Coxeter graph on $I$. Note that $W_{I}$ is a component of $W_{J}$ if and only if $I$ is a component of $J$. We sometimes write $I^{\perp}=\left\{s \in S \mid m_{s t}=2\right.$ for all $\left.t \in I\right\}$.

A root subbasis of $(E,(\cdot, \cdot), \Pi)$ is a triple $(E,(\cdot, \cdot), X)$ (or simply $X$ ) such that $X \subset \Phi^{+}$and for all different $\alpha, \beta \in X$, we have

$$
(\alpha, \beta) \in\left\{-\cos (\pi / m) \mid m \in \mathbb{Z}_{>1}\right\} \cup(-\infty,-1] .
$$

Note that $(E,(\cdot, \cdot), X)$ is indeed a root basis.

We will often consider root bases $(E,(\cdot, \cdot), X)$ where $E$ and $(\cdot, \cdot)$ are fixed but $X$ varies. The objects defined in this section, like $\Phi, U, C$ are then written $\Phi(X)$ etc., in order to stress their dependence on $X$.

1.4.3 Theorem. There exists a bijection between root subbases and root subsystems of $\Phi$ with finitely many canonical generators, defined by $X \mapsto\langle X\rangle$. Moreover, $\left\{r_{\alpha} \mid \alpha \in X\right\}$ is nothing but the canonical set of generators of the reflection group that it generates. For every set of roots $Y$ generating $\langle X\rangle$, we have $\# Y \leq \# X$.

Proof. For the first two statements, see [30], Theorem 4.4. The inequality $\# Y \leq \# X$ is proved in [10], Lemma 1.5.

1.4.4 Lemma. Let $(E,(\cdot, \cdot), X)$ be a root basis, and let $(E,(\cdot, \cdot), Y)$ be a root subbasis. Let $U(X), U(Y)$, respectively, denote their Tits cones. Then $U(X) \subset U(Y)$.

Proof. Let $x \in U(X)$. Let $g \in W$ be such that $x \in g \bar{C}$. Let $W, H$ denote the Coxeter groups associated to $X, Y$, respectively. Let $p, H^{\prime}$ be as in 1.4.2. Since $H$ acts transitively on $H^{\prime}$, we may assume, after translation over an element of $H$, that $g \in p$. Equivalently, for all $\alpha \in Y$, we have $\langle g C, \alpha\rangle \subset \mathbb{R}_{>0}$. Hence $\langle x, \alpha\rangle \geq 0$. Since this is true for all $\alpha \in Y$, we have $x \in C(Y) \subset U(Y)$.

1.4.5 Lemma. Let $\alpha, \beta$ be two roots with $(\alpha, \beta)=-\left(p+p^{-1}\right) / 2, p>0$. Then, after replacing $\alpha, \beta$ by $-\alpha,-\beta$ if necessary, we have

$$
\langle U, \alpha+p \beta\rangle,\langle U, p \alpha+\beta\rangle \subset \mathbb{R}_{\geq 0} .
$$

Proof. Let $X$ be the root subbasis of $\Phi$ generating the same root subsystem as $\{\alpha, \beta\}$ does. It is easy to see that there exists $w \in\left\langle r_{\alpha}, r_{\beta}\right\rangle$ such that $\{w \alpha, w \beta\}=X$ or $-X$. Thus, we may assume that $\{\alpha, \beta\}=X$. Let $u=\alpha+p \beta, v=p \alpha+\beta$, $V=\left\{x \in E^{*} \mid\langle x, u\rangle,\langle x, v\rangle \geq 0\right\}$. First, we show that $r_{\alpha}$ and $r_{\beta}$ fix $V$. We have

$$
r_{\alpha} u=r_{\alpha}(\alpha+p \beta)=-\alpha+p\left(\beta+\left(p+p^{-1}\right) \alpha\right)=p(\beta+p \alpha)=p v,
$$


and since $r_{\alpha}$ is an involution, $r_{\alpha} v=u / p$. This shows that $r_{\alpha}$ fixes $V$, and similarly for $r_{\beta}$. We have $\overline{C(X)} \subset V$, whence $U(X)=\bigcup\left\{w \overline{C(X)} \mid w \in\left\langle r_{\alpha}, r_{\beta}\right\rangle\right\} \subset V$, which proves that $\langle U(X), u\rangle,\langle U(X), v\rangle \subset \mathbb{R}_{\geq 0}$. By 1.4.4, we have $U \subset U(X)$, which finishes the proof.

1.4.6 Definition. We define a (partial) ordering $\leq$ on $\Phi$ by $\alpha \leq \beta \Longleftrightarrow A(\alpha) \subset A(\beta)$.

To each root $\alpha \in \Phi$ we associate open and closed half-spaces in $U^{0}$ by

$$
H(\alpha)=\left\{x \in U^{0} \mid\langle x, \alpha\rangle>0\right\}, \quad K(\alpha)=\left\{x \in U^{0} \mid\langle x, \alpha\rangle \geq 0\right\} .
$$

1.4.7 Proposition. Let $\alpha, \beta$ be two roots. Then the following holds.

(a) If $|(\alpha, \beta)|<1$ then all of the four intersections $A( \pm \alpha) \cap A( \pm \beta)$ are non-empty.

(b) $(\alpha, \beta) \geq 1$ if and only if $\alpha \leq \beta$ or $\beta \leq \alpha$.

(c) If $\alpha \leq \beta$, say (by (b)) $(\alpha, \beta)=\left(p+p^{-1}\right) / 2, p \geq 1$, then for all $x \in U$, we have

$$
\langle x, \alpha\rangle \leq p\langle x, \beta\rangle, \quad\langle x, \alpha\rangle \leq p^{-1}\langle x, \beta\rangle .
$$

(d) $\alpha \leq \beta$ if and only if $\langle U, \beta-\alpha\rangle \subset \mathbb{R}_{\geq 0}$.

(e) Let $X \subset \Phi$ be a root subbasis, and let $\leq_{X}$ denote the ordering on the associated root system $\Phi(X)$. Then for all $\alpha, \beta \in \Phi(X)$, we have $\alpha \leq \beta$ if and only if $\alpha \leq_{X} \beta$

Proof. (a) Let $|(\alpha, \beta)|<1$. Then $P:=\operatorname{Span}\{\alpha, \beta\}$ is positive definite. Hence the group $F$ generated by $r_{\alpha}$ and $r_{\beta}$ is finite, because it is a discrete subgroup of the compact group $\{g \in \mathrm{GL}(E) \mid g P=P,(g x, g y)=(x, y)$ for all $x, y \in E\} \cong$ $\mathrm{O}(2)$. In order to prove that all four intersections are non-empty, suppose the contrary, say $\alpha \leq \beta$. Note that $\alpha<\beta$ because $|(\alpha, \beta)|<1$. Now $r_{\beta} \alpha<r_{\beta} \beta$, whence

$$
\alpha<\beta=-r_{\beta} \beta<-r_{\beta} \alpha=t \alpha,
$$

where $t=r_{\beta} r_{\alpha}$. Hence $\alpha<t \alpha<t^{2} \alpha<\cdots$. Thus the root system generated by $\alpha$, $\beta$ contains infinitely many roots $t^{n} \alpha$, a contradiction. This proves (a).

(b) $\Longrightarrow$. By 1.4.5, the set $\langle U, \alpha-p \beta\rangle$ is contained in $\mathbb{R}_{\geq 0}$ or $\mathbb{R}_{\leq 0}$. In the first case, for all $x \in H(\beta)$, we have $\langle x, \alpha\rangle \geq\langle x, p \beta\rangle>0$, whence $x \in H(\alpha)$, and hence $\beta \leq \alpha$. The other case is treated similarly. $\Longleftarrow$. By (a), we have $|(\alpha, \beta)| \geq 1$. If $(\alpha, \beta) \leq-1$, then, by applying ' $\Longrightarrow$ ' to $\alpha$ and $-\beta$, we find $\alpha \leq-\beta$ or $-\beta \leq \alpha$. It is easy to check that this contradicts the fact that $\alpha \leq \beta$ or $\beta \leq \alpha$. Hence $(\alpha, \beta) \geq 1$.

(c) Same argument as $\Longrightarrow$ in (b).

(d) $\Longrightarrow$. Let $x \in U$. Let $p \geq 1$ be as in (c). Then, by (c), $\langle x, p \beta-\alpha\rangle \geq 0$ and $\langle x, \beta-p \alpha\rangle \geq 0$. Adding these inequalities gives $(p-1)\langle x, \beta-\alpha\rangle \geq 0$. If $p>1$, then the right-hand side of (d) follows. For $p=1$, it follows immediately from (c). $\Longleftarrow$ is trivial. 
(e) $\Longrightarrow$. By (b), we have $(\alpha, \beta) \geq 1$. By (b) again, we have $\alpha \leq_{X} \beta$ or $\beta \leq_{X} \alpha$. Suppose not $\alpha \leq_{X} \beta$. Then, by (d), we have $\langle U(X), \alpha-\beta\rangle \subset \mathbb{R}_{\geq 0}$. Since $U \subset U(X)$ by 1.4.4, we find $\langle U, \alpha-\beta\rangle \subset \mathbb{R}_{\geq 0}$. However, by (d), applied to $\alpha \leq \beta$, we also have $\langle U, \alpha-\beta\rangle \subset \mathbb{R}_{\leq 0}$. Since $U$ contains the open set $C$, it follows that $\alpha=\beta$, whence $\alpha \leq_{X} \beta$, a contradiction. Hence $\alpha \leq_{X} \beta$. $\Longleftarrow$ follows immediately from (d) and the fact that $U \subset U(X), 1.4 .5$.

1.5. Nonnegative matrices. The results of this section will be used in various places in this article, namely in the proofs of 2.2.2, 6.1.1 and B.4.1. See also Section 6.5.

A non-negative, respectively, positive matrix is a square matrix over $\mathbb{R}$ all of whose entries are non-negative, respectively, positive. Similarly for vectors. Let $A=\left(a_{s t}\right)_{s, t \in S}$ be a non-negative matrix. It is called reducible if there exists a partition $S=I \sqcup J(I, J \neq \varnothing)$ such that $a_{s t}=0$ whenever $s \in I, t \in J$. It is called irreducible otherwise.

1.5.1 Theorem (Perron-Frobenius). Let $A$ be an irreducible non-negative matrix. Then up to multiples, A has a unique non-negative eigenvector $v$, say with eigenvalue $\lambda$. The vector $v$ is positive. Moreover, every (complex) eigenvalue $\mu$ satisfies $|\mu| \leq \lambda$, and is simple if $|\mu|=\lambda$.

Proof. [6], 1.4, p. 27.

1.5.2 Lemma. Let $A=\left(a_{i j}\right)$ be a positive definite real square symmetric matrix with $a_{i i}=1$ and $a_{i j} \leq 0(i \neq j)$. Then the entries of $A^{-1}$ are non-negative.

Proof. For a proof that uses the Perron-Frobenius theorem, see [43], 9.1.

Let $E$ be a finite-dimensional real vector space. A cone in $E$ is a subset closed under addition and multiplication by positive scalars. The dimension of a cone is by definition the dimension of its span (the dimension of the empty cone being -1) A cone is called pointed if it does not contain a linear subspace of positive dimension. It is called solid if its interior relative to $E$ is non-empty. A proper cone is a closed pointed solid cone. The theorem of Perron-Frobenius has been generalized to linear maps mapping a proper cone into itself - see [6].

\section{On the Tits cone}

This section shows some results on the Tits cone. Many of the results were proved by Vinberg [47]. 
2.1. The Galois correspondence. Let $A, B$ be sets, and let $R \subset A \times B$ be a relation. The Galois correspondence associated to $R$ is the pair of maps from $P(A)$ to $P(B)$ (power sets) and vice versa, both denoted by a prime, defined as follows. For $X \subset A$,

$$
X^{\prime}=\{y \in B \mid(x, y) \in R \text { for all } x \in X\}
$$

and, similarly, for $Y \subset B$,

$$
Y^{\prime}=\{x \in A \mid(x, y) \in R \text { for all } y \in Y\} .
$$

Usually we write $x^{\prime}$ instead of $\{x\}^{\prime}$. For a set $X$ which is at the same time a subset of $A$ and $B$ (for example, $X=\varnothing$ ), $X^{\prime}$ has two meanings. It will however be clear which one is meant.

We collect a few properties about Galois correspondences. We have $X^{\prime \prime \prime}=X^{\prime}$ for all $X \subset A$ or $X \subset B$. Sets of the form $X^{\prime}$ are called stable. A set $X$ is stable if and only if $X^{\prime \prime}=X$. More generally, $X^{\prime \prime}$ is the smallest stable set containing $X$. We call $X^{\prime \prime}$ the stable closure of $X$. Intersections of stable sets are again stable, for $\bigcap X_{i}^{\prime}=\left(\bigcup X_{i}\right)^{\prime}$. The prime maps set up a bijection between the stable subsets of $A$ and $B$.

The case of our interest is the Galois correspondence associated to the relation $R$ between a Coxeter group $W$ and the Tits cone $U$, defined by

$$
(w, x) \in R \Longleftrightarrow w x=x .
$$

First of all note that stable sets are non-empty. Namely, stable sets in $W$ contain 1 , whereas stable sets in $U$ contain 0 . For every point $x \in U$, the group $x^{\prime}$ is a parabolic subgroup of $W$. Namely, if $x \in w C_{I}$ then $x^{\prime}=w W_{I} w^{-1}$, by 1.2.2(c).

Write $\mu(\alpha)=\{x \in U \mid\langle x, \alpha\rangle=0\}$. We call $\mu(\alpha)$ the wall associated to $\alpha$.

2.1.1 Lemma. Let $x, y \in U$. Then $x^{\prime} \subset y^{\prime}$ if and only if for all roots $\alpha$ : if $x \in \mu(\alpha)$ then $y \in \mu(\alpha)$.

Proof. 'If'. By 1.2.2(c), $x^{\prime}$ and $y^{\prime}$ are generated by the reflections that they contain. We have

$$
r_{\alpha} \in x^{\prime} \Longleftrightarrow x \in \mu(\alpha) \Longrightarrow y \in \mu(\alpha) \Longleftrightarrow r_{\alpha} \in y .
$$

Hence $x^{\prime} \subset y^{\prime}$. 'Only if' is easy.

The following lemma will often be useful.

2.1.2 Lemma. Let $X \subset U$ be a non-empty cone. Then there exists $x \in X$ with $x^{\prime}=X^{\prime}$. 
Proof. Let $x \in X$ be such that $x^{\prime}$ is minimal (such an $x$ exists because if $y \in U$ then $y^{\prime}$ is a parabolic subgroup, and decreasing chains of parabolic subgroups are stable). Suppose not $x^{\prime}=X^{\prime}$. Let $y \in X$ such that $x^{\prime} \not \subset y^{\prime}$, say (by 2.1.1) $y \notin \mu(\alpha)$, $x \in \mu(\alpha)$. By 1.2.2(d), there exists $z \in[x, y]$ such that for every root $\beta$, if $z \in \mu(\beta)$ then $x, y \in \mu(\beta)$. It follows that $z^{\prime} \subset x^{\prime}$ by 2.1.1. Moreover, $z^{\prime} \neq x^{\prime}$ because $r_{\alpha} \in x^{\prime}-z^{\prime}$. Since $X$ is a cone, we have $z \in X$. This contradicts minimality of $x^{\prime}$. Hence $x^{\prime}=X^{\prime}$.

2.1.3 Definition. For $I \subset S$, write $K_{I}=\left\{x \in U \mid\left\langle x, e_{s}\right\rangle=0\right.$ for all $\left.s \in I\right\}$. By a facial subgroup of $W$ we mean a subgroup conjugate to $W_{I}$ for some facial $I \subset S$.

2.1.4 Proposition. A subset of $W$ is stable if and only if it is a facial subgroup. A subset of $U$ is stable if and only if it is of the form $w K_{I}$, with $w \in W, I \subset S$ facial. Finally, the bijection is given by $\left(w W_{I} w^{-1}\right)^{\prime}=w K_{I}$.

Proof. Let $I \subset S$ be facial. From 1.2.2(c) it follows that $\left(K_{I}\right)^{\prime}=W_{I}$. Clearly, $\left(W_{I}\right)^{\prime}=K_{I}$. Translating the results over $w \in W$ gives $\left(w W_{I} w^{-1}\right)^{\prime}=w K_{I}$ and $\left(w K_{I}\right)^{\prime}=w W_{I} w^{-1}$. This proves the 'if' parts. Now we will prove the 'only if' parts. Let $X \subset U$ be stable. By 2.1.2, there exists $x \in X$ with $x^{\prime}=X^{\prime}$. Let $w C_{I}$ be the facet containing $x$. Then $X=X^{\prime \prime}=x^{\prime \prime}=\left(w W_{I} w^{-1}\right)^{\prime}=w K_{I}$. In the last but one equality, 1.2.2 (c) is used. Hence every stable subset in $U$ is of the form $w K_{I}$ (with $I$ facial). Consequently, each stable set in $W$ is of the form $\left(w K_{I}\right)^{\prime}=w W_{I} w^{-1}$ with $I$ facial.

In the special case of the classical root basis, the stable subgroups are precisely the parabolic subgroups. It follows that the intersection of two parabolic subgroups is again parabolic. Thus, every subset $X \subset W$ is contained in a unique smallest parabolic subgroup, which is called the parabolic closure of $X$ and written $\operatorname{Pc}(X)$. For root bases with the property that $\Pi$ is independent, we have $\operatorname{Pc}(X)=X^{\prime \prime}$. However, we will also be considering root bases without this property.

A formula for the intersection of two parabolic subgroups is given by 1.3 .5 (d). Conversely, the intersection of two stable subsets of $U$ is again stable. I do not know of a result on this intersection. In terms of subgroups of $W$, the (equivalent) question is the following.

2.1.5 Question. What is the parabolic closure of the union of two parabolic subgroups?

2.1.6 Theorem. Suppose that $W$ is irreducible and $E=\operatorname{Span}(\Pi)$. Then

$$
U \cap-U= \begin{cases}\{0\} & \text { if } W \text { is infinite }, \\ E^{*} & \text { otherwise. }\end{cases}
$$


Proof. Let $T=U \cap-U$. Then $T$ is a $W$-invariant linear subspace of $E^{*}$. Write $T^{\prime \prime}=w K_{I}$. Since $T$ is $W$-invariant, so is $T^{\prime \prime}$, whence $T^{\prime \prime}=K_{I}$. We will show that

$$
s \in I, m_{s t}>2 \Longrightarrow t \in I \text {. }
$$

Suppose that $s \in I, m_{s t}>2$. Let $x \in K_{I}$. Since $s \in I$, we have $\left\langle x, e_{s}\right\rangle=0$. Since $t T^{\prime \prime}=T^{\prime \prime}$, we have

$$
0=\left\langle t x, e_{s}\right\rangle=\left\langle x, t e_{s}\right\rangle=\left\langle x, e_{s}-2 a_{s t} e_{t}\right\rangle=-2 a_{s t}\left\langle x, e_{t}\right\rangle .
$$

Now $a_{s t}=-\cos \left(\pi / m_{s t}\right) \neq 0$, so $\left\langle x, e_{t}\right\rangle=0$. We conclude $K_{I} \subset K_{t}$. By 2.1.4 we have $W_{t}=\left(K_{t}\right)^{\prime} \subset\left(K_{I}\right)^{\prime}=W_{I}$, whence $t \in I$, which proves (1).

From (1) and irreducibility of $W$ it follows that $I=S$ or $I=\varnothing$. In the first case, we have $T=\{0\}$ and $W$ is infinite by 1.2.6. If $I=\varnothing$, we will show that $W$ is finite and $T=E^{*}$, which will prove the theorem. By 2.1.2, there exists $x \in T$ such that $x^{\prime}=T^{\prime}=T^{\prime \prime \prime}=\left(K_{\varnothing}\right)^{\prime}=W_{\varnothing}=\{1\}$. Hence $x$ is on no wall; we may suppose that $x \in C$. Hence $x \in C \cap-U$. By 1.2.6, $W$ is finite and $U=E^{*}$.

Theorem 2.1.6 has been proved by different methods by Vinberg, [47], Lemma 15, p. 1112 .

\subsection{The interior of the Tits cone}

2.2.1 Definition. Let $I \subset S$ be spherical. Let $E_{I}^{\perp}=\{x \in E \mid$ for all $y \in$ $\left.E_{I}:(x, y)=0\right\}$. Note that $E=E_{I} \oplus E_{I}^{\perp}$. Dually, $E^{*}=Y_{I} \oplus Z_{I}$ where $Y_{I}=\operatorname{Ann}\left(E_{I}^{\perp}\right) \subset E^{*}, Z_{I}=\operatorname{Ann}\left(E_{I}\right) \subset E^{*}$. We define $p_{I}: E \rightarrow E_{I}$, $q_{I}: E^{*} \rightarrow Z_{I}$ to be the projections with respect to these decompositions. Equivalently,

$$
q_{I} x=\frac{1}{\# W_{I}} \sum_{w \in W_{I}} w x .
$$

Note that for all $x \in E^{*}, y \in E$, we have

$$
\langle x, y\rangle=\left\langle x, p_{I} y\right\rangle+\left\langle q_{I} x, y\right\rangle \text {. }
$$

2.2.2 Lemma. Suppose that $\Pi$ is a basis of E. Let $I \subset S$ be spherical.

(a) For all $s \in I, t \in S-I$, we have $\left\langle f_{s}, p_{I} e_{t}\right\rangle \leq 0$.

(b) For all $s \in I, t \in S-I$, we have $\left\langle q_{I} f_{s}, e_{t}\right\rangle \geq 0$.

Proof. (a) Let us write $p_{I} e_{t}=\sum_{s \in I} b_{s} e_{s}$. Then the $b_{s}$ are defined by the equations $\left(e_{u}, p_{I} e_{t}-e_{t}\right)=0(u \in I)$. Thus, $\sum_{s \in I} a_{s u} b_{s}=a_{t u}(u \in I)$, or, in matrix form, $A_{I} b=c$, where $c=\left(a_{t u}\right)_{u \in I}$. On inverting, $b=A_{I}^{-1} c$. By 1.5.2, the entries of $A_{I}^{-1}$ are non-negative. Since $t \notin I$, the entries of $c$ are non-positive. Hence for all $s \in I$ we have $\left\langle f_{s}, p_{I} e_{t}\right\rangle=b_{s} \leq 0$.

(b) This follows from (a) and (2). 
2.2.3 Theorem. Let $I \subset S$ be spherical. Then $q_{I}(C)=C_{I}$.

Proof. Let $D=\bigoplus_{s \in S} \mathbb{R} d_{s}$ be the root basis with $\left(d_{s}, d_{t}\right)=\left(e_{s}, e_{t}\right)$, and let $\pi: D \rightarrow E$ be the linear map mapping $d_{s}$ to $e_{s}$. Let $\pi^{*}: E^{*} \rightarrow D^{*}$ be the dual map defined by $\left\langle\pi^{*} x, y\right\rangle=\langle x, \pi y\rangle$. For $I \subset S$, let $B_{I}$ be what is usually written $C_{I}$, that is,

$$
B_{I}=\left\{x \in D^{*} \mid\left\langle x, d_{s}\right\rangle=0(s \in I),\left\langle x, d_{t}\right\rangle>0(t \in S-I)\right\} .
$$

Let $r_{I}: D^{*} \rightarrow D^{*}$ be the projection, $r_{I} x=\left(\sum_{w \in W_{I}} w x\right) / \# W_{I}$. Since $\pi^{*}$ is a $W$-equivariant map, we have $\pi^{*} \circ q_{I}=r_{I} \circ \pi^{*}$. For all $x \in E^{*}, s \in S$, we have $\left\langle q_{I} x, e_{s}\right\rangle=\left\langle q_{I} x, \pi d_{s}\right\rangle=\left\langle\pi^{*}\left(q_{I} x\right), d_{s}\right\rangle=\left\langle r_{I}\left(\pi^{*} x\right), d_{s}\right\rangle$, which shows

$$
q_{I} x \in \bar{C}_{I} \Longleftrightarrow r_{I}\left(\pi^{*} x\right) \in \bar{B}_{I} .
$$

Furthermore, we have $\left\langle x, e_{s}\right\rangle=\left\langle\pi^{*} x, d_{s}\right\rangle$, which shows that

$$
x \in C \Longleftrightarrow \pi^{*} x \in B:=B_{\varnothing} .
$$

By 2.2.2(b), we have

$$
r_{I}(\bar{B}) \subset \bar{B}_{I}
$$

Hence

$$
x \in C \stackrel{(4)}{\Longleftrightarrow} \pi^{*} x \in B \stackrel{(5)}{\Longrightarrow} r_{I}\left(\pi^{*} x\right) \in \bar{B}_{I} \stackrel{(3)}{\Longleftrightarrow} q_{I} x \in \bar{C}_{I},
$$

whence $q_{I}(C) \subset \bar{C}_{I}$. Since $q_{I} x=x$ for all $x \in \bar{C}_{I} \subset \bar{C}$, we have $q_{I}(\bar{C})=\bar{C}_{I}$. Since $q_{I}$ is linear, $q_{I}(C) \subset C_{I}$. Using the fact that $q_{I}(C)$ is convex and dense in $C_{I}$, we will now show that $q_{I}(C)=C_{I}$. Let $x \in C_{I}$. Let $O_{1}, \ldots, O_{k}$ be open sets in $C_{I}$ such that for every $\left(x_{1}, \ldots, x_{k}\right) \in O_{1} \times \cdots \times O_{k}$, the point $x$ is in the convex hull of $x_{1}, \ldots, x_{k}$. Since $q_{I}(C)$ is dense in $C_{I}$, there exists $x_{i} \in O_{i} \cap q_{I}(C)$ for all $i$. Since $q_{I}(C)$ is convex, we have $x \in q_{I}(C)$. We conclude that $q_{I}(C)=C_{I}$.

2.2.4 Corollary. Every spherical subset of $S$ is facial.

Proof. Let $I \subset S$ be spherical. By 2.2.3, $C_{I}$ is non-empty. Hence $I$ is facial.

For another proof of 2.2.4, see [47], Theorem 7, p. 1114.

We recall that $U^{0}$ denotes the topological interior of $U$ relative to $E^{*}$. Using the fact that $U$ is convex, it is easy to see that so is $U^{0}$. The following corollary was proved by Vinberg [47], Theorem 2 (3).

2.2.5 Corollary. The cone $U^{0}$ equals the union of the facets with finite stabilizer. 
Proof. $\supset$. Let $I \subset S$ be spherical. Recall that (2.2.1)

$$
q_{I} x=\frac{1}{\# W_{I}} \sum_{w \in W_{I}} w x
$$

Using 2.2.3, it follows that $C_{I}$ is contained in $\sum_{w \in W_{I}} w C$. This is a subset of $U$, which is open in $E^{*}$, and hence contained in $U^{0}$. Translation over $w \in W$ shows $w C_{I} \subset U^{0}$.

$\subset$. Let $x \in C_{I} \cap U^{0}$. Let $B \subset U^{0}$ be an open neighbourhood of $x$. We may suppose that $B$ is symmetric around $x$ in the sense that $B=2 x-B$. Since $x \in \bar{C}$, there exists $y \in B \cap C$. Let $z=2 x-y \in B \subset U^{0}$. For $s \in I$, we have $\left\langle x, e_{s}\right\rangle=0$, $\left\langle y, e_{s}\right\rangle>0$, and hence $\left\langle z, e_{s}\right\rangle<0$. Let $w \in W$ such that $z \in w \bar{C}$. By 1.2.2(b), $I \subset\{s \in S \mid \ell(s w)<\ell(w)\}$. By 1.3.7, I is spherical.

\section{The normalizer of a parabolic subgroup}

3.1. The normalizer of a parabolic subgroup. We will describe a result of Deodhar, which will be used more than once in this article (compare 6.8.1). In this section we use it to reduce the conjugacy problem in Coxeter groups to the case $\operatorname{Pc}(v)=\operatorname{Pc}(w)=W$ (3.1.13), assuming we know how to compute parabolic closures.

The part of this section before 3.1.6 follows Cohen [20]. Probably the subsequent results are also known, but perhaps not published.

Recall our notation $\Pi_{I}=\left\{e_{s} \mid s \in I\right\}$. We note that $w_{I} \Pi_{I}=-\Pi_{I}$ for spherical $I \subset S$.

3.1.1 Definition. Let $I \subset S, s \in S-I$. Write $K$ for the connected component of $I \cup\{s\}$ containing $s$. If $K$ is spherical, then we define

$$
v(I, s)=w_{K-\{s\}} w_{K} .
$$

Otherwise, $v(I, s)$ is not defined.

We have

$$
v(I, s)^{-1} \Pi_{I}=\Pi_{J}
$$

for some $J=(I \cup\{s\})-\{t\}, t \in K$.

Consider the directed labelled graph $K$ whose vertices are the subsets of $S$ and in which there is an edge from $I$ to $J$, labelled $s$; each time $v(I, s)$ exists, and $v(I, s)^{-1} \Pi_{I}=\Pi_{J}$.

3.1.2 Proposition. If $I \stackrel{s}{\rightarrow} J$ is a labelled edge of $K$, then so is $J \stackrel{t}{\rightarrow} I$, where either $\{t\}=I-J$, or $I=J$ and $t=s$. 
Proof. Let $I \stackrel{s}{\rightarrow} J$ be a labelled edge. Then $v(I, s)^{-1} e_{s}=-e_{t}$ for some $t \in K$ and $v(I, s)^{-1} e_{u}=e_{f(u)}$ for some bijection $f: I \rightarrow J$. It is easy to see that $v(J, t)$ is defined. Write $I^{\prime}=(J \cup\{t\})-\{s\}$. Then $v(J, t)^{-1} e_{t}=-e_{r}$ for some $r \in K:=I \cup\{s\}$, and $\nu(J, t)^{-1} e_{u}=e_{g(u)}$ for some bijection $g: J \rightarrow J^{\prime}$. It follows that $v(I, s) v(J, t) \in G_{K} \cap W_{K}=\{1\}$. Hence $v(J, t)=v(I, s)^{-1}$. This proves that

$$
J \stackrel{t}{\rightarrow} I
$$

is an edge of $K$. Since $J=(I \cup\{s\})-\{t\}$, we have either $\{t\}=I-J$ or $I=J$ and $t=s$.

3.1.3 Theorem (Deodhar). Let $I, J \subset S, w \in W$ be such that $w^{-1} \Pi_{I}=\Pi_{J}$. Then there exists a directed path

$$
I=I_{0} \stackrel{s_{0}}{\rightarrow} I_{1} \stackrel{s_{1}}{\rightarrow} \cdots \stackrel{s_{t}}{\rightarrow} I_{t+1}=J
$$

in $K$ such that

$$
w=v\left(I_{0}, s_{0}\right) \ldots v\left(I_{t}, s_{t}\right)
$$

and

$$
\ell(w)=\sum_{i=0}^{t} \ell\left(v\left(I_{i}, s_{i}\right)\right) .
$$

Proof. See [27], Proposition 5.5. For finite Coxeter groups, it was proved by Howlett [35], Lemma 4.

For the rest of this section fix $I \subset S$. Let $\mathcal{K}^{0}$ be the connected component of $K$ containing $I$. Let $T$ be a spanning tree of $\mathcal{K}^{0}$. For $J \in T$, let

$$
\mu(J)=v\left(I_{0}, s_{0}\right) \ldots v\left(I_{t}, s_{t}\right)
$$

where

$$
I=I_{0} \stackrel{s_{0}}{\rightarrow} I_{1} \stackrel{s_{1}}{\rightarrow} \cdots \stackrel{s_{t}}{\rightarrow} I_{t+1}=J
$$

is the unique non-reversing path in $T$ from $I$ to $J$. For each labelled edge $e=\left(I_{0} \stackrel{s_{0}}{\rightarrow}\right.$ $\left.I_{1}\right)$, let $\lambda(e)=\mu\left(I_{0}\right) v\left(I_{0}, s_{0}\right) \mu\left(I_{1}\right)^{-1}$. Note that $\lambda(e) \Pi_{I}=\Pi_{I}$.

3.1.4 Definition. Define the group $G_{I}=\left\{w \in W \mid w \Pi_{I}=\Pi_{I}\right\}$.

Note that $G_{I} \subset D_{I I}$ by $1.2 .2(\mathrm{~b})$. We have $G_{I} \cap W_{I}=\{1\}$.

3.1.5 Corollary. $G_{I}$ is generated by all $\lambda(e)$ where e runs through the edges of $\mathcal{K}^{0}$ which are not an edge of $T$. 
Proof. This follows immediately from 3.1.3 and the fact that $\pi_{1}\left(\mathcal{K}^{0}, I\right)$ is generated by the paths in $\mathcal{K}^{0}$ from $I$ to $I$ containing exactly one edge not in $T$.

3.1.6 Proposition. Let $I, J \subset S, g \in D_{\varnothing J}$ such that $g^{-1} W_{I} g=W_{J}$. Then $\Pi_{I}=g \Pi_{J}$. In particular, $g \in D_{I J}$.

Proof. By 1.4.3, there exists a unique set $X \subset \Phi^{+}$such that

(1) $\left\{r_{\alpha} \mid \alpha \in X\right\}$ generates $W_{I}$;

(2) for all different $\alpha, \beta \in X$ :

$$
(\alpha, \beta) \in\left\{-\cos (\pi / m) \mid m \in \mathbb{Z}_{>1}\right\} \cup(-\infty,-1] .
$$

Note that $X$ is nothing else but $\Pi_{I}$. We will show that $g \Pi_{J}$ shares these properties with $\Pi_{I}$. Since $g \in D_{\varnothing J}$, we have $g \Pi_{J} \subset \Phi^{+}$. Since $g^{-1} W_{I} g=W_{J}$, the set $g \Pi_{J}$ satisfies (1). It obviously satisfies (2) as well. By unicity of $X$, we conclude that $g \Pi_{J}=\Pi_{I}$.

3.1.7 Corollary. Let $I, J \subset S$. Then $W_{I}$ and $W_{J}$ are conjugate in $W$ if and only if $I$ and $J$ are in the same connected component of $K$.

Proof. 'If' is easy. In order to prove 'only if', let $g^{-1} W_{I} g=W_{J}$. By replacing $g$ by $g w$ for an appropriate $w \in W_{J}$, we may suppose that $g \in D_{\varnothing J}$. By 3.1.6, $g^{-1} \Pi_{I}=\Pi_{J}$. By 3.1.3, $I$ and $J$ are in the same connected component of $K$.

We write $N\left(W_{I}\right), Z\left(W_{I}\right)$ for the normalizer and centralizer, respectively, in $W$ of $W_{I}$.

3.1.8 Definition. Denote the natural group homomorphism $G_{I} \rightarrow \operatorname{Sym}(I)$ by $\phi$. By $\psi$ we denote the (unique) map $N\left(W_{I}\right) \rightarrow W$ such that $\{\psi(w)\}=w W_{I} \cap D_{\varnothing, I}$.

3.1.9 Proposition. (a) The group $N\left(W_{I}\right)$ equals the semi-direct product $G_{I} \ltimes W_{I}$, the natural projection $N\left(W_{I}\right) \rightarrow G_{I}$ being $\psi$.

(b) $\operatorname{ker} \phi \psi \subset Z\left(W_{I}\right) W_{I}$.

Proof. (a) Note that $G_{I} \cap W_{I}=1$ and $G_{I}$ normalizes $W_{I}$. Thus $G_{I}$ and $W_{I}$ generate a semi-direct product $G_{I} \ltimes W_{I}$, which we denote by $N$. We will prove $N=N\left(W_{I}\right)$. $\subset$ is clear. $\supset$. Let $w \in N\left(W_{I}\right)$. Write $\{g\}=w W_{I} \cap D_{\varnothing, I}$. Note that $g \in N\left(W_{I}\right)$. Thus, by 3.1.6, $g \in G_{I}$. This proves $N=N\left(W_{I}\right)$. From the foregoing, it also follows that $\psi$ is the natural projection.

(b) Let $w \in \operatorname{ker} \phi \psi$. Then $\psi(w) e_{s}=e_{s}$ for all $s \in I$, which implies $\psi(w) \in$ $Z\left(W_{I}\right)$. Hence $w \in \psi(w) W_{I} \subset Z\left(W_{I}\right) W_{I}$. 
3.1.10 Remark. The reverse inclusion of 3.1 .9 (b) does not necessarily hold. It is well known that for the Coxeter group of type $A_{n}$ (with generating set $S=\{1, \ldots, n\}$, and $m_{s t}=2$ if $|s-t|>1$ and $m_{s t}=3$ if $|s-t|=1$ ), the longest element maps $e_{s}$ to $-e_{n+1-s}$. Using this fact, we will give a counterexample to the reverse inclusion. Let $(W, S)$ be the Coxeter system given by the Coxeter graph of Figure 2, where we denote $S=\{1, \ldots, 6\}$. Define subsets $I, J, K \subset S$ by $I=\{1,2\}$, $J=\{1,2,3,4,6\}, K=\{1,2,4,5,6\}$. Let $w=w_{J} w_{K} w_{I}$. We have $w e_{1}=-e_{1}$, $w e_{2}=-e_{2}$, whence $w \in Z\left(W_{I}\right)$. Furthermore, $w w_{I}=w_{J} w_{K}$ interchanges $e_{1}$ and $e_{2}$. This shows that $w w_{I}=\psi(w)$ and $w \notin \operatorname{ker} \phi \psi$.

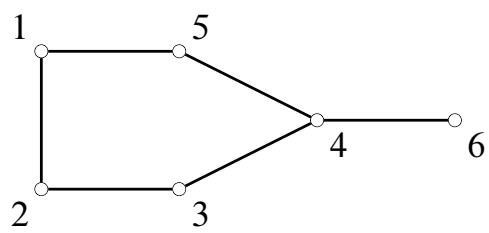

Figure 2. The Coxeter graph considered in 3.1.10.

3.1.11 Corollary. $Z\left(W_{I}\right) W_{I}$ has finite index in $N\left(W_{I}\right)$, and there exists an algorithm finding $g_{1}, \ldots, g_{k} \in N\left(W_{I}\right)$ such that

$$
N\left(W_{I}\right)=\bigsqcup_{i} g_{i} Z\left(W_{I}\right) W_{I} .
$$

Proof. Finiteness of the index follows immediately from 3.1.9(b) and the fact that $\operatorname{Im} \phi \psi \subset \operatorname{Sym}(I)$ is finite.

The algorithm is as follows. By 3.1.5, we know how to find generators $h_{1}, \ldots, h_{\ell}$ of $G_{I}$. Next compute $f_{i}=\psi h_{i}$. Write $F=\phi G_{I}$. Then $F$ is generated by the $f_{i}$ and is contained in the finite group $\operatorname{Sym}(I)$. Hence it is possible to compute the elements of $F$. By remembering how elements of $F$ are written as products of the $f_{i}$, we find $k_{i} \in G_{I}$ with the property $G_{I}=\bigsqcup_{i} k_{i} \operatorname{ker} \phi$. Consequently, $N\left(W_{I}\right)=$ $\bigsqcup_{i} k_{i} \operatorname{ker} \phi \psi$. Since $\operatorname{ker} \phi \psi \subset Z\left(W_{I}\right) W_{I}$, we have $N\left(W_{I}\right)=\bigcup_{i} k_{i} Z\left(W_{I}\right) W_{I}$. Lemma 3.1.12 enables us to compute a maximal set $X \subset\{1,2, \ldots, \ell\}$ such that for all different $i, j \in X$, we have $k_{i}^{-1} k_{j} \notin Z\left(W_{I}\right) W_{I}$, that is, $N\left(W_{I}\right)=\bigsqcup_{i \in X} k_{i} Z\left(W_{I}\right) W_{I}$.

3.1.12 Lemma. Let $g \in N\left(W_{I}\right)$. Then $g \in Z\left(W_{I}\right) W_{I}$ if and only iffor each connected component $J$ of $I$, we have either $\psi(g) e_{s}=e_{s}$ for all $s \in J$, or $J$ is spherical and $\psi(g) e_{s}=-w_{J} e_{s}$ for all $s \in J$.

Proof. Since $\psi(g) \in g W_{I}$, we have $g \in Z\left(W_{I}\right) W_{I} \Longleftrightarrow \psi(g) \in Z\left(W_{I}\right) W_{I}$. Hence we may suppose that $\psi(g)=g$, that is, $g \in G_{I}$. The 'if' part is easy. In order to 
prove the 'only if' part, suppose that $g \in G_{I} \cap Z\left(W_{I}\right) W_{I}$, say $g=z w, z \in Z\left(W_{I}\right)$, $w \in W_{I}$. For all $s \in I$, we have $z e_{s}=\lambda_{s} e_{s}$ where $\lambda_{s} \in\{-1,1\}$. For all $s, t \in S$ we have $\left(e_{s}, e_{t}\right)=\left(z e_{s}, z e_{t}\right)=\lambda_{s} \lambda_{t}\left(e_{s}, e_{t}\right)$. If $s$ and $t$ are adjacent in the Coxeter graph (that is, $m_{s t}>2$ ), it follows that $\lambda_{s}=\lambda_{t}$. Hence $\lambda_{s}=\lambda_{t}$ whenever $s$ and $t$ are in the same connected component of $S$. Let $J \subset I$ be a connected component of $I$, and let $\bar{w}$ be the projection of $w \in W_{I}$ on $W_{J}$. Then $g \Phi_{J}=z w \Phi_{J}=z \Phi_{J}=\Phi_{J}$. Since also $g \in G_{I}$, it follows that $g \in G_{J}$. The first case to be considered is $z e_{S}=e_{S}$ for all $s \in J$. Then $z \in G_{J}$, so $w=z^{-1} g \in G_{J}$, and $\bar{w} \in G_{J} \cap W_{J}=\{1\}$. It follows that for all $s \in J$, we have $g e_{s}=z \bar{w} e_{s}=z e_{s}=e_{s}$. The second case is $z e_{s}=-e_{s}$ for all $s \in J$. Then $\bar{w} \Pi_{J}=-\Pi_{J}$. By 1.2.6, $W_{J}$ is finite and $\bar{w}=w_{J}$. It follows that $g e_{s}=z \bar{w} e_{s}=z w_{J} e_{s}=-w_{J} e_{s}$.

3.1.13 Corollary. The conjugacy problem for Coxeter groups can be solved as soon as for all Coxeter groups the following two problems have been solved.

(1) Given $w \in W$, determine $I \subset S, g \in W$ such that $\operatorname{Pc}(w)=g W_{I} g^{-1}$.

(2) Given $v, w \in W$ such that $\operatorname{Pc}(v)=\operatorname{Pc}(w)=W$, decide whether $v, w$ are conjugate in $W$.

Proof. The algorithm is as follows. First use (1) to compute $I, J, g, h$ such that $\operatorname{Pc}(v)=g W_{I} g^{-1}, \operatorname{Pc}(w)=h W_{J} h^{-1}$. If $I$ and $J$ are not in the same connected component of $K$, then $v$ and $w$ are certainly not conjugate by 3.1.7. If $I$ and $J$ are in the same connected component of $K$, we may conjugate $v$ and $w$ so as to ensure $\operatorname{Pc}(v)=\operatorname{Pc}(w)=W_{I}$. Next compute $\left\{g_{i}\right\}$ such that $N\left(W_{I}\right)=\bigsqcup_{i} g_{i} Z\left(W_{I}\right) W_{I}$ (3.1.11). We claim that $v$ and $w$ are conjugate if and only if $v$ and $g_{i}^{-1} w g_{i}$ are conjugate in $W_{I}$ for some $i$.

'If' is trivial. Suppose that $v=g^{-1} w g$. Then $W_{I}=\operatorname{Pc}(v)=\operatorname{Pc}\left(g^{-1} w g\right)=$ $g^{-1} \operatorname{Pc}(w) g=g^{-1} W_{I} g$, that is, $g \in N\left(W_{I}\right)$. Hence we may write $g=g_{i} z h$, $z \in Z\left(W_{I}\right), h \in W_{I}$. Hence $v=h^{-1} z^{-1} g_{i}^{-1} w g_{i} z h=h^{-1} g_{i}^{-1} w g_{i} h$, that is, $v$ and $g_{i}^{-1} w g_{i}$ are conjugate in $W_{I}$. This problem was assumed solvable in (2).

3.2. Finite subgroups of Coxeter groups. In this section we give a solution to the conjugacy problem for torsion (that is, finite order) elements of a Coxeter group. The results in this section are well known. The reflection representation of a Coxeter group supplies a test whether a given element of the group is torsion or not.

3.2.1 Proposition. (a) Let $H \subset W$ be a finite subgroup. Then $\operatorname{Pc}(H)$ is finite. Moreover, $H^{\prime \prime}=\operatorname{Pc}(H)$.

(b) There exists an algorithm which, given a finite subgroup $H \subset W$, determines $g$, I such that $\operatorname{Pc}(H)=g W_{I} g^{-1}$.

Proof. (a) Choose any $x \in U^{0}$. Let $y=\sum_{h \in H} h x$. Then $y$ is $H$-invariant, that is, $H \subset y^{\prime}$. But since $y \in U^{0}, y^{\prime}$ is a finite parabolic subgroup by 2.1.4 and 2.2.5, thus 
proving the first statement. The latter statement follows from the fact that spherical subsets of $S$ are facial (2.2.4).

(b) The proof of (a) suggests the following algorithm. Consider the classical root basis. Choose any $x \in U^{0}$, for example, $x=\sum_{s \in S} f_{s}$. Compute $y=\sum_{h \in H} h x$, so that $\operatorname{Pc}(H) \subset y^{\prime}$. Since $y \in U$, it is possible to find $g \in W, I \subset S$ such that $y \in g C_{I}$, which implies that $y^{\prime}=g W_{I} g^{-1}$. Since $y \in U^{0}, y^{\prime}$ is finite. Since intersections of parabolic subgroups are again parabolic subgroups, $\operatorname{Pc}(H)$ is a parabolic subgroup of the Coxeter group $y^{\prime}$. Hence the problem to find $\operatorname{Pc}(H)$ is now a finite one.

3.2.2 Proposition. There exists an algorithm deciding whether two torsion elements in $W$ are conjugate.

Proof. The proof is similar to that of 3.1.13. Let $v, w \in W$ be torsion. By 3.2.1 (b), we know how to compute $I, J, g, h$ such that $\operatorname{Pc}(v)=g W_{I} g^{-1}, \operatorname{Pc}(w)=h W_{J} h^{-1}$. By 3.1.7, we can decide whether $W_{I}$ and $W_{J}$ are conjugate. If not, then $v$ and $w$ are certainly not conjugate. Now assume that $\operatorname{Pc}(v)=\operatorname{Pc}(w)=W_{I}$. Let $N\left(W_{I}\right)=$ $\bigsqcup_{i} g_{i} Z\left(W_{I}\right) W_{I}$. Now $v$ and $w$ are conjugate in $W$ if and only if there exists $i$ such that $v$ and $g_{i}^{-1} w g_{i}$ are conjugate in $W_{J}$, which is a finite problem.

Combination of 3.2.1 and the Galois correspondence gives the following result.

3.2.3 Lemma. (a) Let $F \subset \Phi$ be a finite root subsystem, such that $F=\operatorname{Span}(F) \cap \Phi$. Then $F$ is a parabolic root subsystem.

(b) Let $P \subset E$ be a positive definite linear subspace such that $P=\operatorname{Span}(P \cap \Phi)$. Then $P \cap \Phi$ is a finite parabolic root subsystem.

Proof. (a) Write $H=\left\langle r_{\alpha} \mid \alpha \in F\right\rangle$. We may assume that $H^{\prime \prime}=W$ and $E=$ Span $\Pi$. Note that $H$ is finite by 1.2.6. Hence so is $W$ by 3.2.1. Since all $m_{s t}$ are finite, our root basis is the classical root basis modulo some subspace. Again by 1.2.6, our root basis is the classical one, and $U=E^{*}$. Let Ann denote the annihilator maps for $E$ and $E^{*}$. We have

$$
\text { Span } F=\text { Ann Ann } F=\text { Ann } H^{\prime}=\text { Ann } H^{\prime \prime \prime}=\operatorname{Ann} W^{\prime}=\operatorname{Ann}\{0\}=E,
$$

whence $F=\Phi \cap \operatorname{Span} F=\Phi$.

(b) By 1.2.5, the root system $\Phi \cap P$ is finite. The result now follows from (a).

\section{Hyperbolic reflection groups}

The conjugacy problem for affine Coxeter groups is treated in Section 4.2. In the subsequent sections, we will solve the conjugacy problem for the so-called hyperbolic reflection groups. Section 4.3 is devoted to the definitions of the involved notions. 
4.1. Signatures. A real vector space equipped with a quadratic form is said to have signature $(k, \ell, m)$ if it is isomorphic to $\mathbb{R}^{k+\ell+m}$ with the quadratic form

$$
Q\left(x_{1}, \ldots, x_{k}, y_{1}, \ldots, y_{\ell}, z_{1}, \ldots, z_{m}\right)=\left(x_{1}^{2}+\cdots+x_{k}^{2}\right)-\left(y_{1}^{2}+\cdots+y_{\ell}^{2}\right) .
$$

By Sylvester's theorem, $k, \ell$ and $m$ exist uniquely. By signature $(k, \ell)$ we mean signature $(k, \ell, 0)$. It is easy to prove that a real vector space with a quadratic form of signature $(k, \ell, m)$ has a subspace of signature $\left(k^{\prime}, \ell^{\prime}, m^{\prime}\right)$ if and only if $k^{\prime} \leq k$, $\ell^{\prime} \leq \ell, k^{\prime}+m^{\prime} \leq k+m$ and $\ell^{\prime}+m^{\prime} \leq \ell+m$.

4.2. Affine Coxeter groups. An affine Coxeter group is an infinite Coxeter group associated to a root basis $(E,(\cdot, \cdot), \Pi)$ such that $E$ has signature $(n, 0,1)$ for some $n$.

It is known that every irreducible affine Coxeter group is what is known as an affine Weyl group, [12], Proposition 2, p. 146. In particular, it is a semi-direct product $\mathbb{Z}^{n} \rtimes W$ for some Weyl group $W$, which is a finite Coxeter group itself. We remark here that the product of two affine Coxeter groups is again affine. The easiest proof is by using the semi-direct decompositions as above - the direct sum of the two root bases does not work.

4.2.1 Proposition. The conjugacy problem for affine Coxeter groups is solvable in linear time.

Proof. Let $\widetilde{W}$ be an affine Coxeter group. As noted above, we have a semi-direct decomposition $\widetilde{W}=T \rtimes W, T \cong \mathbb{Z}^{n}, W$ a finite (Coxeter) group. Moreover, an isomorphism $T \rightarrow \mathbb{Z}^{n}$ and a set of representatives $R$ of $\widetilde{W} / T$ can be computed. Thus, it remains to check if there are $t \in t, r \in R$ such that

$$
t v t^{-1}=r w r^{-1} \text {. }
$$

Since $R$ is finite, we may suppose $r$ to be fixed. Write $v=t_{1} r_{1}, r w r^{-1}=t_{2} r_{2}$, $t_{i} \in T, r_{i} \in R$. We have $t v t^{-1}=t t_{1}\left(r_{1} t^{-1} r_{1}^{-1}\right) r_{1}=\left(t r_{1} t^{-1} r_{1}^{-1}\right) t_{1} r_{1}$, so (6) is equivalent to $r_{1}=r_{2}$ and

$$
\left(t r_{1} t^{-1} r_{1}^{-1}\right) t_{1}=t_{2} \text {. }
$$

Consider the homomorphism $f: T \rightarrow T, t \mapsto t r_{1} t^{-1} r_{1}^{-1}$. Equation (7) is solved by computing the image of $f$ and checking whether it contains $t_{2} t_{1}^{-1}$.

As to complexity, computation of $t_{1}, r_{1}, t_{2}, r_{2}$ can be done in linear time; since there are only finitely many maps of the form $f$ above, we may suppose them to be computed once and for all. More precisely, for a given $f$, there are group homomorphisms $p_{i}: T \rightarrow \mathbb{Z} / d_{i}$ such that $f=\bigcap_{i} \operatorname{ker} p_{i}$. This shows that a membership test for ker $f$ is logarithmic in time. Thus, the complexity is linear. 
4.3. Hyperbolicity and word hyperbolicity. A hyperbolic reflection group is a Coxeter group associated to some root basis $(E,(\cdot, \cdot), \Pi)$ such that $E$ has signature $(n, 1)$ for some $n$. This definition differs from the definitions used by many authors in that we do not require the group to have finite covolume in hyperbolic space. The methods of this section apply to all hyperbolic reflection groups in our sense.

Roughly speaking, a group is called word hyperbolic if it admits a finite presentation such that every word mapping to the identity in the group contains strictly more than half of a relation. We will now state this definition more precisely.

Let $G$ be a group and let $X \subset G$ be a generating subset. Let $F(X)$ denote the free group on $X$. Let $\pi: F(X) \rightarrow G$ denote the unique homomorphism with $\pi(x)=x$ for all $x \in X$. Let $\ell: F(X) \rightarrow \mathbb{N}$ denote the length function with respect to $X$.

A group $G$ is called word hyperbolic if it admits a finite presentation $\langle X, R\rangle$ such that, with the above notation, for all $w \in \pi^{-1}(1)$, there are $a, b, p, q \in F(X)$ such that $a b \in R, \ell(a b)=\ell(a)+\ell(b), \ell(b)<\ell(a), w=p a q, \ell(w)=\ell(p)+\ell(a)+\ell(q)$.

There exist equivalent definitions of word hyperbolicity, which relate the geometry of the Cayley graph of $G$ to the geometry of the hyperbolic plane. For these definitions of word hyperbolicity we refer to [1], [22]. There it is also shown that word hyperbolicity does not depend on $X$, that is, if $G$ is word hyperbolic, then for every finite generating set $X$ a presentation $\langle X, R\rangle$ as above exists.

The following theorem classifies word hyperbolic Coxeter groups.

4.3.1 Theorem (Moussong). Let $(W, S)$ be a Coxeter system, with $S$ finite. The following assertions are equivalent.

(1) $W$ is word hyperbolic.

(2) W has no subgroups isomorphic to $\mathbb{Z}^{2}$.

(3) There is no $I \subset S$ such that $W_{I}$ is irreducible affine of rank at least 3 , and there are no disjoint $I, J \subset S$ such that the subgroups $W_{I}$ and $W_{J}$ commute and are infinite.

Proof. See [43], Theorem 17.1. Note that (2) $\Longrightarrow$ (3) is easy. We also mention the easy result that word hyperbolic groups have no subgroups isomorphic to $\mathbb{Z}^{2}$, whence $(1) \Longrightarrow(2)$.

4.3.2 Remark. A hyperbolic reflection group is not necessarily word hyperbolic, nor does the converse implication necessarily hold, namely, that every word hyperbolic Coxeter group is isomorphic to some hyperbolic reflection group. A counterexample to the first implication is the group given by the Coxeter graph of Figure 3. It is not word hyperbolic since it contains the affine group of type $\widetilde{A}_{2}$. However, the quadratic form associated to its classical representation has signature $(3,1)$. Two counterexamples for the converse implication (with the beautiful additional property that the Davis-Moussong complex is a topological manifold) are given by Moussong [43], p. 47. 


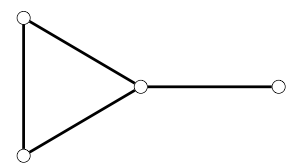

Figure 3. The Coxeter graph considered in 4.3.2.

In [32] it is proved that there is a linear solution to the conjugacy problem for word hyperbolic groups.

4.4. Removed. This section has been removed from the original thesis, but its number is retained here for easy reference.

4.5. Hyperbolic space. Let $E$ be a real vector space equipped with a symmetric bilinear form $(\cdot, \cdot)$ of signature $(n, 1)$. Sometimes we write $Q(x)=(x, x)$. Define $H=\{x \in E \mid(x, x)<0\}$. Note that $H$ has two connected components; we denote these by $H^{+}, H^{-}$. Also note that $H^{+}$and $H^{-}$are convex. Let $S=\{x \in E \mid$ $(x, x)=0, x \neq 0\}$, which has two components $S^{+}=S \cap \overline{H^{+}}, S^{-}=S \cap \overline{H^{-}}$. Let $\mathrm{O}(n, 1)=\{g \in \mathrm{GL}(E) \mid(g x, g y)=(x, y)$ for all $x, y \in E\}$. Define $\mathrm{O}^{+}(n, 1)$ to be the subgroup of $\mathrm{O}(n, 1)$ of all elements which preserve $H^{+}$. Clearly, $\mathrm{O}(n, 1)=$ $\mathrm{O}^{+}(n, 1) \times\{1,-1\}$. Define $P S^{n}=\left\{\mathbb{R}_{>0} x \mid x \in E-\{0\}\right\}$, and let $\pi: E-\{0\} \rightarrow P S^{n}$ be the projection. Now hyperbolic $n$-space is defined to be $\mathbb{H}^{n}=\pi H^{+} \subset P S^{n}$. We call $S^{n-1}=\pi S^{+}$the $(n-1)$-sphere. It is the boundary in $P S^{n}$ of hyperbolic $n$-space. Elements of $S^{n-1}$ are called points at infinity.

The group $\mathrm{O}^{+}(n, 1)$ acts transitively on $\mathbb{H}^{n}$, and the stabilizer of any point is isomorphic to $\mathrm{O}(n)$. There exists an $\mathrm{O}^{+}(n, 1)$-invariant Riemannian metric on $\mathbb{H}^{n}$, which is unique up to multiples. For $x, y \in H^{+}$, there exists a unique shortest path from $\pi x$ to $\pi y$. It is $\pi[x, y]$, which is simply denoted by $[\pi x, \pi y]$. The length of this path is called the distance between $\pi x$ and $\pi y$, notation $d(\pi x, \pi y)$. A formula for $d(\pi x, \pi y)$ is

$$
d(\pi x, \pi y)=|\log r|,
$$

where $r$ is either of the two (real, positive) roots of

$$
\frac{4(x, y)^{2}}{(x, x)(y, y)}=r+2+r^{-1} .
$$

Although hyperbolic geometry is happening in $\pi H^{+}$, we prefer to consider $H^{+}$. Having this in mind, we write $d(x, y)=d(\pi x, \pi y)$ for $x, y \in H^{+}$.

Since we are going to solve the conjugacy problem for reflection groups in $\mathrm{O}^{+}(n, 1)$, it is natural to classify conjugacy classes in $\mathrm{O}^{+}(n, 1)$ first. 
4.5.1 Proposition. Let $g \in \mathrm{O}^{+}(n, 1)$. Then precisely one of the following statements is true.

(a) g has a fixed point in $\mathbb{H}^{n}$.

(b) g has no fixed points in $\mathbb{H}^{n}$ and has exactly one fixed point at infinity.

(c) g has no fixed points in $\mathbb{H}^{n}$ and has exactly two fixed points at infinity.

Proof. See [5], Proposition A.5.14.

4.5.2 Definition. In the situation of 4.5.1, $g$ is called elliptic, parabolic, hyperbolic when (a), (b), (c), respectively, holds.

4.6. Hyperbolic reflection groups. For the rest of this section let $(E,(\cdot, \cdot), \Pi)$ be a root basis of signature $(n, 1)$, so that $W$ is a hyperbolic reflection group. Probably the assumption that $\Pi$ is finite is not necessary for many results.

Since $E$ is non-degenerate, it may be identified with $E^{*}$ by $x \mapsto(x, \cdot)$. We retain the notations of Sections 1.2 and 4.5.

4.6.1 Proposition. The set $U \cap H$ equals one of the components $H^{+}, H^{-}$.

Proof. In [41], Corollary 1.3, p. 82, it is proved that $U$ contains one of the components. By 2.1.6, $U$ does not meet the other component.

In view of the above proposition assume from now on that $U \cap H=H^{+}$.

4.6.2 Corollary. There exists an algorithm that, when given an element of $\mathrm{O}^{+}(n, 1)$, decides whether it is in $W$.

Proof. Let $g \in \mathrm{O}^{+}(n, 1)$ be given. Fix $z \in C \cap H^{+}$. Now $g z \in H^{+} \subset U$. Hence there exists $w \in W$ such that $g z \in w \bar{C}$. By 1.2 .4 we can find such a $w$. We claim that $g \in W$ if and only if $g=w$. In order to prove 'only if', let $g \in W$. Since $g z \in w \bar{C} \cap g C$, we have, by 1.2.2(c), $g=w$, which proves the claim. This condition is easy to verify, which finishes the algorithm.

4.6.3 Question. An open question is how to extend Corollary 4.6.2 to every root basis $(E,(\cdot, \cdot), \Pi)$, that is, how to decide, when given an element $g \in \operatorname{GL}(E)$, whether $g \in W$. Let $G \subset \operatorname{GL}(E)$ be the subgroup of matrices of determinant \pm 1 . Let us fix a basis of $E$, and for $g \in G$ let us denote by $N(g)$ the maximum of the absolute values of the entries of the matrix of $g$ with respect to this basis. Since $W$ is a discrete subset of $G$ and $N^{-1}([0, t])$ is compact for all $t \in \mathbb{R}$, there exists a function $f: \mathbb{R} \rightarrow \mathbb{R}$ such that for all $g \in W$ we have $\ell(g) \leq f(N(g))$. Hence, our question has an affirmative answer if a computable function $f$ with the above property exists. 
4.6.4 Definition. Define $D_{I}=C_{I} \cap H^{+}, D=D_{\varnothing}=C \cap H^{+}$.

4.6.5 Proposition. Let $I \subset S$. Then $D_{I} \neq \varnothing \Longleftrightarrow I$ is spherical.

Proof. $\Longrightarrow$. Let $x \in D_{I}$. Then $W_{I}$ is a discrete subgroup of the stabilizer in $\mathrm{O}^{+}(n, 1)$ of $x$. Since $(x, x)<0$, this stabilizer is isomorphic to $\mathrm{O}(n)$, that is, a compact group. Hence $W_{I}$ is finite. $\Longleftarrow$. Choose $x \in D=C \cap H^{+}$. Then $q_{I} x \in C_{I}$ by 2.2.3. Since $H^{+}$is convex, and by $2.2 .1, q_{I} x \in H^{+}$. Hence $q_{I} x \in D_{I}$.

4.7. The conjugacy problem. Retain the setting of Section 4.6.

4.7.1 Definition. We define the map $\phi: U \rightarrow \bar{C}$ by $\{\phi(x)\}=W x \cap \bar{C}$.

4.7.2 Definition. Let $g \in \mathrm{O}^{+}(n, 1)$ be parabolic (see 4.5.2). By $R_{g}$ we will denote the intersection of $S^{+}$with the eigenspace corresponding to the fixed point at infinity. Strictly speaking, $R_{g}$ is the fixed point at infinity. For hyperbolic $g$, the axis is defined to be the set of $x \in \mathbb{H}^{n}$ such that $d(x, g x)$ is minimal.

4.7.3 Proposition. Let $w \in W$ be a parabolic element. Then $R_{w} \subset U$.

Proof. The proof consists of three steps.

Step 1: For all $\varepsilon>0$ there exists $x \in H^{+}$such that $d(x, w x)<\varepsilon$.

Proof. With respect to a certain basis of $E$, we have

$$
\begin{aligned}
w & =\left(\begin{array}{cccc}
1 & 2 & 1 & 0 \\
0 & 1 & 1 & 0 \\
0 & 0 & 1 & 0 \\
0 & 0 & 0 & A
\end{array}\right), \\
Q\left(x_{1}, \ldots, x_{n}\right) & =\left(x_{2}^{2}-x_{1} x_{3}\right)+\left(x_{4}^{2}+\cdots+x_{5}^{2}\right) .
\end{aligned}
$$

Let $x=(a, 0,1,0, \ldots)$. Then $w x=(a+1,1,1,0, \ldots)$. We have $(x, x)=-a$, $(x, w x)=-(2 a+1) / 2,(w x, w x)=(x, x)=-a$. By Section 4.5, $d(x, w x)=$ $|\log r|$, where $r$ is either of the two roots of

$$
\frac{4(x, w x)^{2}}{(x, x)(w x, w x)}=\left(\frac{2 a+1}{a}\right)^{2}=r+2+r^{-1} .
$$

Clearly, if $a$ approaches infinity, then $r$ approaches 1, which proves Step 1 .

Step 2: For $I \subset S$, define

$$
\varepsilon(I)=\inf \left\{\sum_{s \in I} d\left(x, D_{s}\right) \mid x \in D\right\} .
$$

Here, $D_{s}=D_{\{s\}}$ from 4.6.4. 
If $\varepsilon(I)=0$ then there exists $y \in \bar{C}_{I}$ with $(y, y) \leq 0, y \neq 0$.

Proof. Let $\omega$ be the invariant Riemannian metric on $\mathbb{H}^{n}$. Let $\eta$ be any Riemannian metric on a neighbourhood in $P S^{n-1}$ of $\overline{\mathbb{H}^{n}}$ such that $\eta \leq \omega$ on $\mathbb{H}^{n}$. Such an $\eta$ exists, because some model of $\mathbb{H}^{n}$ looks as follows [4], p. 49:

$$
\mathbb{H}^{n}=\left\{x \in \mathbb{R}^{n}|| x \mid<1\right\} \subset \mathbb{R}^{n} \cup\{\infty\}=P S^{n}, \quad \omega=\frac{|d x|}{1-|x|^{2}} .
$$

Thus, $\eta$ may be chosen to be $\eta=|d x| \leq \omega$. Let $d_{0}$ be the corresponding distance. We have $d_{0}(x, y) \leq d(x, y)$ for all $x, y \in \mathbb{H}^{n}$.

Now suppose that $\varepsilon(I)=0$. For each integer $k>0$, choose $x_{k} \in \pi(D)$ such that

$$
\sum_{s \in I} d\left(x_{k}, \pi\left(D_{s}\right)\right) \leq \frac{1}{k}
$$

Let $K$ be the closure in $P S^{n-1}$ of $\pi(D)$. Since $K$ is compact, the $x_{k}$ have a limit point $x \in K$. Since

$$
\sum_{s \in I} d_{0}\left(x_{k}, \pi\left(D_{s}\right)\right) \leq \sum_{s \in I} d\left(x_{k}, \pi\left(D_{s}\right)\right) \leq \frac{1}{k},
$$

it follows that

$$
\sum_{s \in I} d_{0}\left(x, \pi\left(D_{s}\right)\right)=0
$$

in other words, $x \in \overline{\pi\left(D_{s}\right)}$ for all $s \in I$. Hence $y$ has the desired properties whenever $x=\mathbb{R}_{>0} y$, which concludes Step 2 .

Step 3: End of the proof.

Let

$$
\varepsilon=\frac{1}{\# S} \min \{\varepsilon(I) \mid I \subset S, \varepsilon(I) \neq 0\} .
$$

Let $x \in H^{+}$be such that $d(x, w x)<\varepsilon$ (existing by step 1). After conjugating $w$, we may suppose that $x \in \bar{C}$. Consider the curve $\phi[x, w x]$, where $\phi$ is the map defined in 4.7.1. This is a closed curve in $\bar{C}$ of length $d(x, w x)$. Let

$$
I=\left\{s \in S \mid \phi[x, w x] \cap D_{s} \neq \varnothing\right\} .
$$

Note that $w \in W_{I}$. Furthermore, we have $\varepsilon(I)=0$ since otherwise

$$
\varepsilon(I) \leq \sum_{s \in I} d\left(x, D_{s}\right) \leq(\# I) d(x, w x)<(\# I) \varepsilon \leq(\# S) \varepsilon \leq \varepsilon(I),
$$

which is a contradiction. By Step 2, there exists $y \in \bar{C}_{I}$ with $(y, y) \leq 0, y \neq 0$. Since $y \in \bar{C}_{I}$ and $w \in W_{I}$, we have $w y=y$. But in 4.5.1 parabolic elements $w$ of $\mathrm{O}^{+}(n, 1)$ were characterized as elements having no fixed points in $\mathbb{H}^{n}$, and exactly one in $S^{n-1}$, which is $R_{w}$. Hence $R_{w}=\mathbb{R}_{>0} y \subset \bar{C}_{I} \subset U$. 
4.7.4 Remark. A result analogous to proposition 4.7.3 is known for every discrete group in $\mathrm{O}^{+}(2,1)$ (that is, not only reflection groups), see [4], Corollary 9.2.9, p. 216. Probably this result generalizes to every discrete group in $\mathrm{O}^{+}(n, 1)$.

4.7.5 Definition. Let $K$ be a subfield of $\mathbb{R}$. We say that a root basis $(E,(\cdot, \cdot), \Pi)$ is defined over $K$ if there exists a basis $x_{1}, x_{2}, \ldots, x_{n}$ of $E$ such that $\left(x_{i}, x_{j}\right) \in K$ for all $i, j$, and $\Pi \subset \sum_{i} K x_{i}$.

4.7.6 Theorem. Let $W$ be a finite rank hyperbolic reflection group defined over a real number field. Then the conjugacy problem for $W$ is solvable.

Proof. For algorithms on calculations in number fields see Section 5.9. We will describe the algorithm that decides if $v, w \in W$ are conjugate. First calculate the matrices of $v$ and $w$ and decide if they are conjugate in $\mathrm{O}^{+}(n, 1)$. If not, we are done, so suppose they are. Next decide which class $v$ and $w$ fall into elliptic, parabolic or hyperbolic (see 4.5.2). These three cases are treated separately.

Case 1: $v$ and $w$ are elliptic. Now $v$ and $w$ have finite order. To see this, note that the topological closure in $\mathrm{O}^{+}(n, 1)$ of $w^{\mathbb{Z}}$ is compact. Since $w^{\mathbb{Z}}$ is discrete, it follows that $w$ has finite order, as has $v$. But for finite order elements, the conjugacy problem has been solved in 3.2.2.

Case 2: $v$ and $w$ are parabolic. By 4.7.3, $R_{v}, R_{w} \subset U$. Hence we can find $x, y \in W, I, J \subset S$ such that $R_{v} \subset x C_{I}, R_{w} \subset y C_{J}$. If $I \neq J, v$ and $w$ are not conjugate, so suppose that $I=J$. After conjugating $v$ and $w$ by suitable elements of $W$, we may suppose that $R_{v}, R_{w} \subset C_{I}$. Note that $v, w \in W_{I}$, and $g v g^{-1}=w \Longrightarrow g R_{v}=R_{w} \Longleftrightarrow g \in W_{I}$. Hence $v$ and $w$ are conjugate if and only if they are conjugate in $W_{I}$. Note that $\operatorname{Span} \Pi_{I}$ has signature $(k, 0,1)$ for some $k$, because $\Pi_{I} \perp R_{w}$ and $w \in W_{I}$ is not elliptic. Hence $W_{I}$ is an affine Coxeter group. Now Case 2 is finished by 4.2.1.

Case 3: $v$ and $w$ are hyperbolic. The key here is the axis of an hyperbolic element. This idea is very similar to our solution to the conjugacy problem for the case $\operatorname{Pc}(v)=\operatorname{Pc}(w)=W$ irreducible, infinite, non-affine, which is treated in Section 6.7. We refer to 6.7.5 for an adaptation to our case.

\section{The axis}

5.1. Introduction. When solving the conjugacy problem for a group $G$, it is natural to look for the shortest elements in a conjugacy class $C(g)$ (with respect to a finite generating subset $X)$. A particular nice case is if $C(g)$ contains straight elements $h$, that is, $\ell\left(h^{n}\right)=|n| \ell(h)$ for all $n \in \mathbb{Z}$. Torsion (that is finite order) elements different from 1 are examples of elements not conjugate to any straight element. 
In Section 5.4 we define a metric $d_{\Phi}$ on $U^{0}$ such that for all $x, y \in W$, we have $d_{\Phi}(x C, y C)=d_{W}(x, y)$. The axis $Q(w)$ is then defined to be the set of those $x \in U^{0}$ such that $d\left(x, w^{n} x\right)=n d(x, w x)$ for all $n>0$. This generalizes the straight elements. The advantage is that $Q(w)$ is nonempty for all $w \in W$.

The action of $W$ on $\Phi$ is also important. For given $w \in W$, we will in Section 5.2 classify roots into three classes, called $w$-periodic, $w$-even and $w$-odd roots. In Section 5.7 a refinement of this classification is made.

Two applications are that the parabolic closure $\operatorname{Pc}(w)$ of $w$ is generated by the so-called $w$-essential reflections (5.8.3, see 5.7.5 for a definition of essential), and a cubic algorithm computing the parabolic closure (5.10.9).

\subsection{Periodic, even and odd roots}

5.2.1 Definition. Let $w \in W$, and let $A \subset W$ be a half-space. We call $A w$-periodic or simply periodic if there exists $n>0$ such that $w^{n} A=A$.

5.2.2 Proposition. Let $w \in W$, and let $A \subset W$ be a half-space. Then exactly one of the following holds.

(1) A is periodic.

(2) $A$ is not periodic and the number $\#\left\{n \in \mathbb{Z} \mid A\right.$ separates $w^{n} x$ from $\left.w^{n+1} x\right\}$ is finite and even for every $x \in W$.

(3) $A$ is not periodic and the number $\#\left\{n \in \mathbb{Z} \mid A\right.$ separates $w^{n} x$ from $\left.w^{n+1} x\right\}$ is finite and odd for every $x \in W$.

Proof. Let $A$ be a half-space which is not periodic.

For $x, y \in W$ let $L(x, y)$ denote the set of $n \in \mathbb{Z}$ such that $A$ separates $w^{n} x$ from $w^{n} y$. We must show that $L(x, w x)$ is finite and that the parity of $\# L(x, w x)$ does not depend on $x$ (only $A$ ).

Note that $L(x, y)$ is also the set of $n \in \mathbb{Z}$ such that $w^{-n} A$ separates $x$ from $y$. But there are only finitely many half-spaces separating $x$ from $y$. If $L(x, y)$ were infinite, it would follow that there are distinct integers $m, n$ such that $w^{-m} A=w^{-n} A$, contradicting the fact that $A$ is not periodic. So $L(x, y)$ is finite for all $x, y \in W$.

If $X, Y$ are sets, write $X \oplus Y:=(X-Y) \cup(Y-X)$. We have $L(x, y)=L(y, x)$ and

$$
L(x, y) \oplus L(y, z)=L(x, z) \text { for all } x, y, z \in W .
$$

We find

$$
\begin{aligned}
L(x, w x) \oplus L(y, w y) & =(L(x, w x) \oplus L(w x, y)) \oplus(L(w x, y) \oplus L(y, w y)) \\
& =L(x, y) \oplus L(w x, w y),
\end{aligned}
$$

which has even cardinality because $L(w x, w y)$ has the same cardinality as $L(x, y)$. It follows that the parity of $\# L(x, w x)$ does not depend on $x$. 
5.2.3 Definition. In cases (2) and (3) in 5.2.2, we call $A$ even and $o d d$, respectively.

In order to express the relation to $w$, we will sometimes speak about $w$-even half-spaces, etc. The characterization of 5.2.2 is also valid for roots and reflections.

5.2.4 Corollary. The characterization of 5.2.2 is conjugacy covariant, that is, for all $w, g$, A, we have

$$
A \text { is } w \text {-even } \Longleftrightarrow g A \text { is } g w g^{-1} \text {-even, }
$$

and similarly for odd.

Proof. Write $S(A, w, x)=\left\{n \in \mathbb{Z} \mid A\right.$ separates $w^{n} x$ from $\left.w^{n+1} x\right\}$. It is readily seen that $S(A, w, x)=S\left(g A, g w g^{-1}, g x\right)$. Let $A$ be even. Then for all $x \in W$, we have $\# S\left(g A, g w g^{-1}, x\right)=\# S\left(A, w, g^{-1} x\right)$ is finite and even. Hence $g A$ is $g w g^{-1}$-even. The converse and the case of odd $A$ are similar.

5.2.5 Examples. (a). For $w$ of finite order, all half-spaces are periodic.

(b). Consider the universal Coxeter group $(W, S)$, that is, all $m_{s t}$ are infinite. Then the Cayley graph is a tree. Let $w \in W$ be cyclically reduced, that is, $w=s_{1} s_{2} \ldots s_{n}$, $s_{i} \in S, s_{i} \neq s_{i+1}, s_{n} \neq s_{1}$. Let $x_{k} \in W(k \in \mathbb{Z})$ be defined by $x_{k}=s_{1} s_{2} \ldots s_{k}$ $(1 \leq k \leq n)$ and $x_{k+n}=w x_{k}$ for all $k$. Then a half-space is odd if and only if it is one of the two half-spaces separating $x_{k}$ from $x_{k+1}$ for some $k$. There are no periodic half-spaces.

(c). Consider the affine Coxeter group $W$ of Figure 4. Elements of $W$ correspond to triangles, and walls with lines in the figure. The identity element is marked $\varnothing$, and

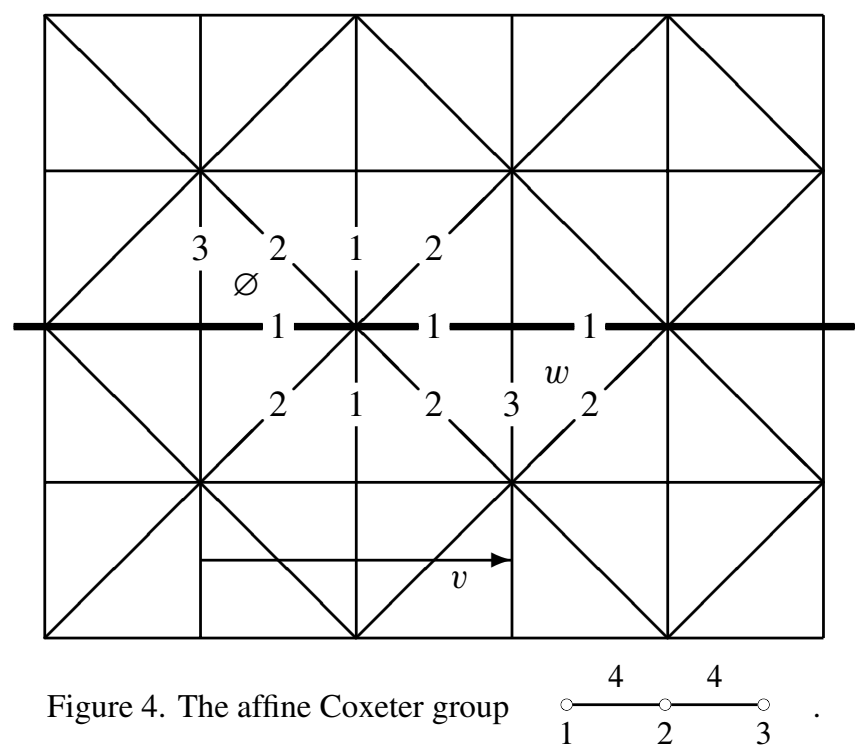


some edges $\{v, v s\}$ have been labelled $s$. Consider the element $w=12123$. It is easy to see that $w$ is a glide-reflection, that is, a reflection through the thick line, followed by a translation over the vector $v$. All horizontal lines define periodic walls; the other walls are odd. We will come back to this example a few times.

5.2.6 Lemma. There are only finitely many $w^{\mathbb{Z}}$-orbits of odd half-spaces.

Proof. Let $A$ be an odd half-space. Since odd integers are non-zero, there exists $n \in \mathbb{Z}$ such that $A$ separates $w^{n}$ from $w^{n+1}$. Hence $w^{-n} A$ separates 1 from $w$. But there are only finitely many half-spaces separating 1 from $w$, which proves the lemma.

5.2.7 Lemma. For all $w \in W, n>0$, a half-space is $w$-odd if and only if it is $w^{n}$-odd, and similarly for even.

Proof. Left to the reader.

5.3. Distinguishing even roots from odd ones. Whereas in the previous section we considered half-spaces, from now on we will prefer roots over half-spaces. The translation between these two is provided by Section 1.4, especially 1.4.7.

Write $c=\max (2, \#\{(\alpha, \beta) \mid \alpha, \beta \in \Phi\} \cap(-1,1)), r=\# S$. Note that $c$ is finite by 3.2 .1 (a).

A sequence $a_{1}, a_{2}, \ldots$ of complex numbers is said to satisfy a linear recurrence of order $k$ if there are $p_{0}, \ldots, p_{k} \in \mathbb{C}\left(p_{0}, p_{k} \neq 0\right)$ such that $p_{0} a_{n}+\cdots+p_{k} a_{n+k}=$ 0 for all $n$. If this is the case, then the sequence is completely determined by $k$ consecutive entries and $p_{0}, \ldots, p_{k}$.

5.3.1 Lemma. There exists a constant $N=N(W)$ such that the following holds. For each $w \in W, \alpha \in \Phi$, there exists $n$ such that $1 \leq n \leq N$ and $\left|\left(\alpha, w^{n} \alpha\right)\right| \geq 1$. We may take $N=c^{r}$.

Proof. Write $a_{n}=\left(\alpha, w^{n} \alpha\right)$ and note that $a_{-n}=a_{n}$. Let $K$ be the smallest positive integer such that $\left|a_{K}\right| \geq 1$, or infinity if such an $a_{K}$ does not exist. Consider the $r$-tuples $\left(a_{n+1}, a_{n+2}, \ldots, a_{n+r}\right)$ such that $-K<n+1, n+r<K$. If all $r$-tuples are different, then, since there are at most $c^{r}$ possible $r$-tuples, we have $2 K-r \leq c^{r}$, whence $K \leq\left(c^{r}+r\right) / 2$. If two $r$-tuples are equal, then, since the sequence $a_{n}$ satisfies a linear recurrence of order $r$, the sequence is periodic. The period is at most $c^{r}$, which shows that for some $n$ with $1 \leq n \leq c^{r}$, we have $a_{n}=a_{0}=(\alpha, \alpha)=1$. In both cases we have $\left|a_{n}\right| \geq 1$ for some $n$ with $1 \leq n \leq \max \left(\left(c^{r}+r\right) / 2, c^{r}\right)=c^{r}$.

5.3.2 Proposition. There exists a constant $N=N(W)$ such that for all $w \in W$ the following holds. Let $\alpha$ be w-odd. 
(a) For all $n \geq 1:\left(\alpha, w^{n} \alpha\right)>-1$.

(b) There exists $n, 1 \leq n \leq N$ : $\left(\alpha, w^{n} \alpha\right) \geq 1$.

Let $\alpha$ be $w$-even.

(c) For all $n \geq 1:\left(\alpha, w^{n} \alpha\right)<1$.

(d) There exists $n, 1 \leq n \leq N$ : $\left(\alpha, w^{n} \alpha\right) \leq-1$.

We may take $N$ to be the constant of 5.3.1.

Proof. Write $A=A(\alpha)$. First, we prove (a) and (c). In order to prove (a), let $\alpha$ be odd, say $w^{k} \in A$ for $k>0$ big enough. Then there exists $k>0$ with $w^{k}, w^{k-n} \in A$. Hence $A \cap w^{n} A \neq \varnothing$, and, similarly, $W-A$ and $W-w^{n} A$ are not disjoint. In other words, neither of the sets $A$ and $W-w^{n} A$ is contained in the other. By 1.4.7 (b), we find $\left(\alpha,-w^{n} \alpha\right)<1$, which proves (a). In order to prove (c), suppose $\alpha$ to be even but $\left(\alpha, w^{n} \alpha\right) \geq 1$. By 1.4.7(b), we have, after replacing $\alpha$ by $-\alpha$ if necessary, $A \subset w^{n} A$. Since $A$ is not periodic, there exists $x \in w^{n} A-A$. Since $\cdots \subset w^{-n} A \subset A \subset w^{n} A \subset \cdots$, we find that $x \in w^{k n} A \Longleftrightarrow k>0$. Hence $\alpha$ is odd, a contradiction, which proves (c).

Now we will prove (b) and (d). Let $\alpha$ be either odd or even. Let $n$ be such that $1 \leq n \leq N$ and $\left|\left(\alpha, w^{n} \alpha\right)\right| \geq 1$. Such an $n$ exists by 5.3.1. If $\alpha$ is odd, then by (a) we have $\left(\alpha, w^{n} \alpha\right) \geq 1$, which proves (b). If $\alpha$ is even, then by (c) we have $\left(\alpha, w^{n} \alpha\right) \leq-1$, which proves (d).

We refer to 5.10 .1 for an algorithm, based on 5.3.2, to decide whether a root is even or odd.

5.4. A pseudometric on $U^{0}$. We turn attention to the interior $U^{0}$ of $U$. By 2.2.5, it equals the union of the facets with finite stabilizer.

For a root $\alpha$, let $f_{\alpha}: U^{0} \rightarrow\{-1 / 2,0,1 / 2\}$ be the map

$$
f_{\alpha}(x)= \begin{cases}-1 / 2 & \text { if }\langle x, \alpha\rangle<0 \\ 0 & \text { if }\langle x, \alpha\rangle=0 \\ 1 / 2 & \text { if }\langle x, \alpha\rangle>0\end{cases}
$$

Recall that a pseudometric on a set $X$ is a map $d: X \times X \rightarrow \mathbb{R}_{\geq 0}$ such that for all $x, y, z \in X$ one has $d(x, z) \leq d(x, y)+d(y, z)$. We define pseudometrics $d_{\alpha}$ and $d_{\Phi}$ on $U^{0}$ by

$$
d_{\alpha}(x, y)=\left|f_{\alpha}(x)-f_{\alpha}(y)\right|, \quad d_{\Phi}(x, y)=\sum_{\alpha \in \Phi^{+}} d_{\alpha}(x, y) .
$$

Let $x, y \in U^{0}$. A root $\alpha$ is said to separate $x$ from $y$ if $d_{\alpha}(x, y)>0$. We say that $\alpha$ strictly separates $x$ from $y$ if $d_{\alpha}(x, y)=1$. 
For $x \in U^{0}$, the set $\left\{y \in U^{0} \mid d_{\Phi}(x, y)=0\right\}$ equals the facet containing $x$. Thus, the facets in $U^{0}$ form a metric space. By 2.2.4 and 2.2.5, this metric space is purely combinatorial, that is, up to isometry, it depends only on the Coxeter system, not on the root basis. In fact, the facets in $U^{0}$ are in bijection with the union over the spherical $I \subset S$ of $W / W_{I}$, by the identification $w C_{I} \mapsto w W_{I}$.

5.4.1 Lemma. (a) For all $v, w \in W$ we have $d_{\Phi}(v C, w C)=d_{W}(v, w)$.

(b) For all $x, y \in U^{0}$, the distance $d_{\Phi}(x, y)$ is finite.

Proof. (a) This follows from the fact that $w \mapsto w C$ is a bijection, and $\alpha$ separates $v$ from $w$ if and only if it separates every point of $v C$ from every point of $w C$.

(b) Let $I \subset S$ be spherical. Then $d_{\Phi}\left(C_{I}, C\right)$ is finite (more precisely, it is half the number of reflections in $\left.W_{I}\right)$. Using (a), $d_{\Phi}\left(v C_{I}, w C_{J}\right) \leq d_{\Phi}\left(C_{I}, C\right)+$ $d_{\Phi}(v C, w C)+d_{\Phi}\left(C_{J}, C\right)$ is finite.

Recall that $\mu(\alpha)=\left\{x \in U^{0} \mid\langle x, \alpha\rangle=0\right\}=K(\alpha)-H(\alpha)$. We write $\mu(A)$, $H(A), K(A)$ for $\mu(\alpha), H(\alpha), K(\alpha)$, where $A=A(\alpha)$.

The following lemma translates the language of the Tits cone into the language of the Coxeter group, and will frequently be used. The proof is left to the reader.

5.4.2 Lemma. Let $g \in W$ and let $A \subset W$ be a half-space. The following are equivalent.

(1) $g \in A$.

(2) $g C \subset H(A)$.

(3) $g C \subset K(A)$.

(4) $g \bar{C} \subset K(A)$.

5.5. The axis. We now come to the definition of an object that will be the center of study in this section.

5.5.1 Definition. For each $w \in W$, define the axis $Q(w)$ to be

$$
\left\{x \in U^{0} \mid \text { for all } n \in \mathbb{Z}: d_{\Phi}\left(x, w^{n} x\right)=|n| d_{\Phi}(x, w x)\right\} .
$$

5.5.2 Examples. (a) $Q(w)$ contains the fixed points in $U^{0}$ of $w$. It is easy to see that when there is at least one such fixed point (equivalently, $w$ is torsion by 2.2 .5 and 3.2.1), then $Q(w)$ equals the set of fixed points.

(b) Let us consider again the universal Coxeter group of 5.2.5 (b). Then all facets have codimension 0 or 1 . The facets of codimension 0 are in bijection with $W$, those of codimension 1 with the edges in the Cayley graph. In the notation of 5.2.5 (b), $Q(w)$ equals $\left(\bigcup_{k \in \mathbb{Z}} x_{k} C\right) \cup\left(\bigcup_{k \in \mathbb{Z}} x_{k} C_{s_{k}}\right)$. 
(c) In the example of 5.2.5(c), $Q(w)$ equals the thick line. The proof is left to the reader. The reader may wish to use $(1) \Longrightarrow(2)$ of 5.5 .4 below, applied to a root associated to the thick line.

(d) Let $X \subset E^{*}$ be a $w$-invariant two-dimensional subspace, on which $w$ has positive real eigenvalues. Then $X \cap U^{0}$ is contained in $Q(w)$. A particular case is where $W$ is affine, $w \in W$ a translation, and $X=\operatorname{Span}\{x, w x\}$ for some $x \in U^{0}$. It follows that in this case, $Q(w)=U^{0}$.

(e) In Figures 5, 6 and 7, we give a picture of $Q(w)$ for three cases, following the conventions of 5.2.5 (c). All three examples have the property that $W$ can be made to act (cocompactly) discretely on the hyperbolic plane. Otherwise, drawing pictures would become difficult. The fundamental regions are triangles (in Figures 5 and 6) and a 4-gon (in Figure 7). The 4-gon has three right angles and an angle equal to $\pi / 3$. The pictures are constructed in such a way that the action of $w$ on the plane of the picture is a translation or a glide-reflection, according to whether $\ell(w)$ is even or odd. Moreover, we have drawn the pictures in such a way that $Q(w)$ is an open strip, as shown in the pictures. As an example, one wall is dotted in each figure. It has the property that one of the associated roots $\alpha$ is minimal under the condition $Q(w) \subset H(\alpha)$.

Denote the centralizer in $W$ of $w$ by $Z(w)$.

5.5.3 Lemma. For all $g, w \in W$, we have $Q\left(g w g^{-1}\right)=g Q(w)$. In particular, $Q(w)$ is invariant under $Z(w)$.

Proof. Left to the reader.

5.5.4 Lemma. Let $w \in W, x \in U^{0}$. The following assertions are equivalent.

(1) $x \in Q(w)$.

(2) For every $\alpha \in \Phi$, the function $\mathbb{Z} \rightarrow\{-1 / 2,0,1 / 2\}, n \mapsto f_{\alpha}\left(w^{n} x\right)$, is monotonic.

Proof. Statement (1) is equivalent to saying that for all $k, \ell, m \in \mathbb{Z}, k<\ell<m$, we have

$$
d_{\Phi}\left(w^{k} x, w^{m} x\right)=d_{\Phi}\left(w^{k} x, w^{\ell} x\right)+d_{\Phi}\left(w^{\ell} x, w^{m} x\right)
$$

This is equivalent to saying that for all $\alpha \in \Phi, k, \ell, m \in \mathbb{Z}, k<\ell<m$, we have

$$
d_{\alpha}\left(w^{k} x, w^{m} x\right)=d_{\alpha}\left(w^{k} x, w^{\ell} x\right)+d_{\alpha}\left(w^{\ell} x, w^{m} x\right) .
$$

This, in turn, is equivalent to (2).

The following lemma gives some more properties of the function of 5.5.4 (2). 


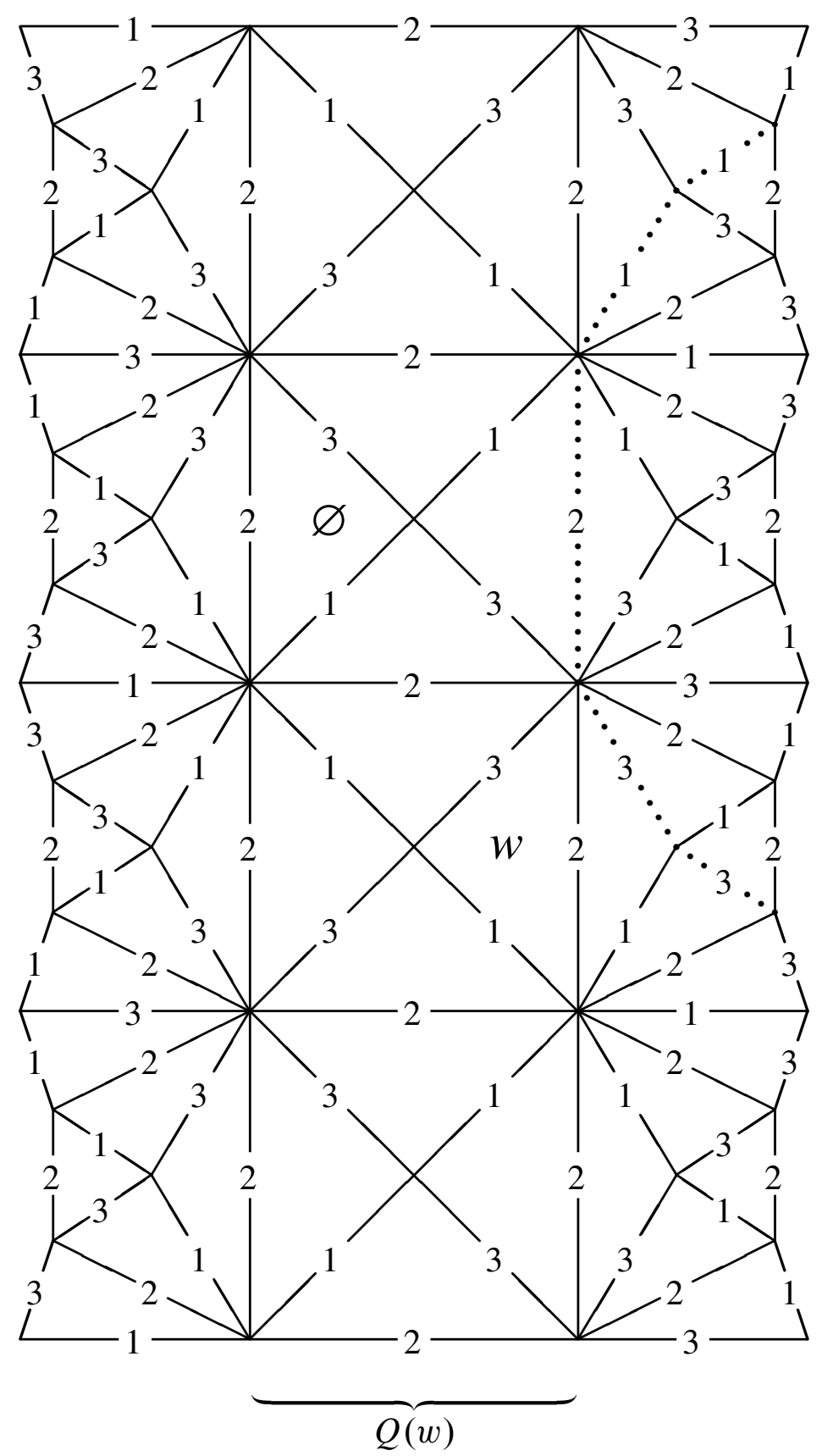

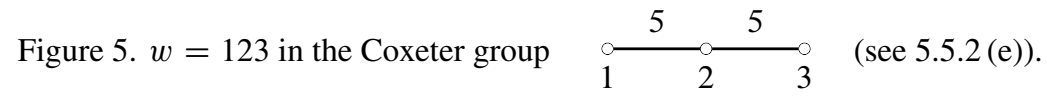




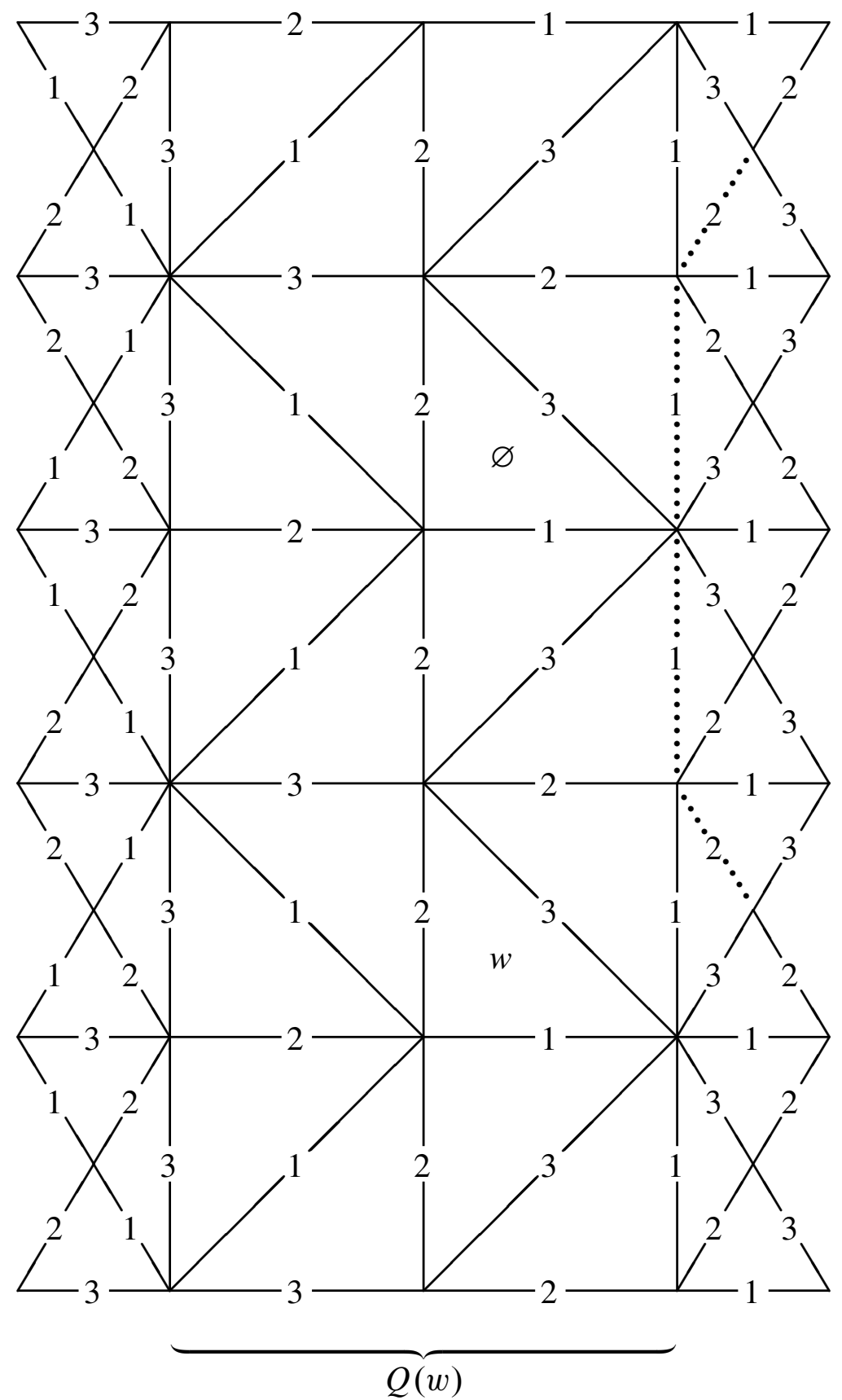

Figure 6. $w=1232, m_{12}=m_{23}=3, m_{13}=4$. 


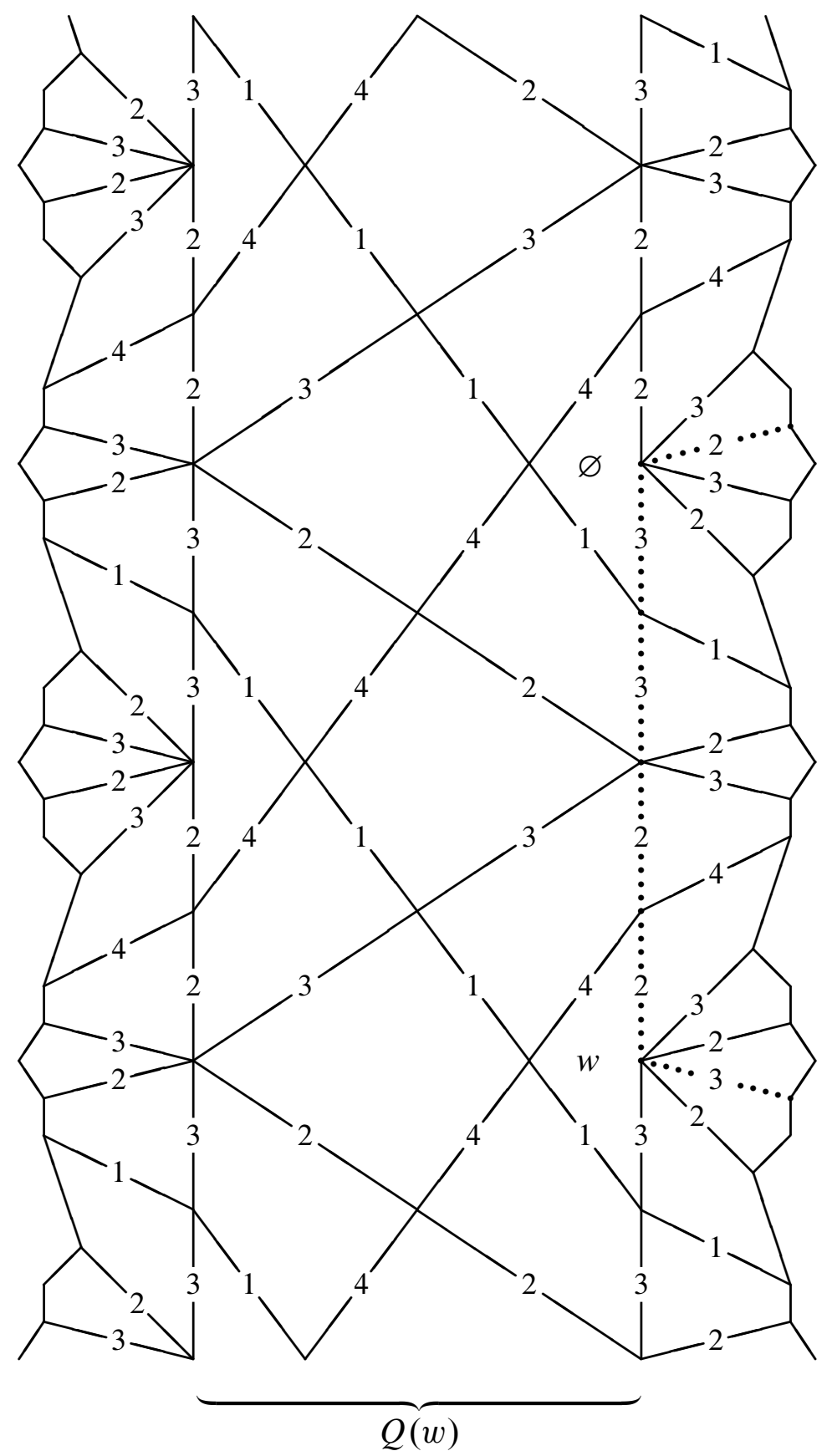

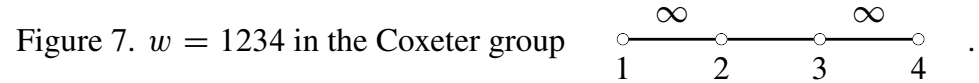


5.5.5 Lemma. Let $w \in W, x \in U^{0}, \alpha \in \Phi$ and write $g: \mathbb{Z} \rightarrow\{-1 / 2,0,1 / 2\}$, $g(n)=f_{\alpha}\left(w^{n} x\right)$.

(a) Suppose that $g$ is monotonic. If $g$ is not constant, then its image contains $\{-1 / 2,1 / 2\}$. Furthermore, $g$ is not constant if and only if $\alpha$ is odd.

(b) Suppose that $\alpha$ is not odd. Then $g$ is monotonic if and only if it is constant.

Proof. (a) Suppose that $g$ is not constant, but the image of $g$ does not contain $\{-1 / 2,1 / 2\}$. Then after replacing $w$ by $w^{-1}$ if necessary, there exists $N \in \mathbb{Z}$ such that for all $n>N$, we have $g(n)=0$, or, equivalently, $\left\langle w^{n} x, \alpha\right\rangle=0$. Since the sequence $\left\langle w^{n} x, \alpha\right\rangle(n \in \mathbb{Z})$ satisfies a linear recurrence, it follows that $\left\langle w^{n} x, \alpha\right\rangle=0$ for all $n$ and $g$ is constant, a contradiction. This proves the first statement.

In order to prove the latter statement, write $A=A(\alpha)$ and choose $y \in W$ such that $x \in y \bar{C}$. We have

$$
g(n)=1 / 2 \Longrightarrow w^{n} y \in A, \quad g(n)=-1 / 2 \Longrightarrow w^{n} y \notin A .
$$

Suppose that $g$ is not constant. Then (8) and the fact that $g$ is monotonic immediately imply that $A$ is odd. Now suppose $g$ to be constant but $A$ odd. Then from (8) it follows that $g$ is constant zero. Hence the walls $\mu\left(w^{n} \alpha\right)$ all pass through $x \in U^{0}$. But there are only finitely many walls through a point in $U^{0}$. Hence $\alpha$ is periodic, contradicting the fact that $A$ is odd. This concludes the proof of (a).

(b) Easy and left to the reader.

5.5.6 Definition. Let $\alpha$ be a root. Then we call $\alpha$ outward if for some (hence all) $x \in U^{0}$ the following holds. For almost all $n \in \mathbb{Z}$, we have $n\left\langle w^{n} x, \alpha\right\rangle<0$. It is called inward if $-\alpha$ is outward. Similarly for half-spaces. By 5.2.2, $\alpha$ is odd if and only if $\alpha$ or $-\alpha$ is outward. The set of outward roots is denoted $\operatorname{Out}(w)$. We define $r(w)$ to be $\#\left(w^{\mathbb{Z}} \backslash \operatorname{Out}(w)\right)$. So $r(w)$ is finite by 5.2.6.

The following proposition gives another characterization of $Q(w)$. Our membership test for $Q(w)$ (5.10.5) will be based on it.

5.5.7 Proposition. For all $x \in U^{0}$, we have $d_{\Phi}(x, w x) \geq r(w)$, with equality if and only if $x \in Q(w)$. In particular, $r(w) \leq \ell(w)$.

Proof. Let $x \in U^{0}$. Write $r=r(w)$ and let $\alpha_{1}, \ldots, \alpha_{r}$ be outward roots such that $\operatorname{Out}(w)$ equals the disjoint union over $i$ of $w^{\mathbb{Z}} \alpha_{i}$. Then for every outward root $\alpha$, by 5.5.5, we have $\sum_{n \in \mathbb{Z}} d_{\alpha}\left(w^{n} x, w^{n+1} x\right) \geq 1$, whence

$$
d_{\Phi}(x, w x)=\sum_{\alpha \in \Phi^{+}} d_{\alpha}(x, w x) \geq \sum_{i=1}^{r} \sum_{n \in \mathbb{Z}} d_{\alpha_{i}}\left(w^{n} x, w^{n+1} x\right) \geq r(w) .
$$

Equality holds if and only if $x \in Q(w)$, by 5.5.4 and 5.5.5. 
5.5.8 Lemma. Let $\alpha$ be even. Then $Q(w) \subset H(\alpha)$ or $Q(w) \subset H(-\alpha)$ (or both).

Proof. Write $A=A(\alpha)$. Let $g \in W$. By replacing $\alpha$ by $-\alpha$ if necessary, we may suppose that $w^{n} g \in A$ for almost all $n \in \mathbb{Z}$. We will show that $Q(w) \subset H(\alpha)$. Let $x \in Q(w)$. Let $h \in W$ be such that $x \in h \bar{C}$. We will show that $w^{n} h \in A$ for almost all $n \in \mathbb{Z}$. If not, infinitely many $w^{-n} A$ separate $g$ from $h$. Thus, $A$ is periodic, a contradiction. Hence $w^{n} h \in A$ for almost all $n \in \mathbb{Z}$. Hence, $w^{n} x \in K(\alpha)$ for almost all $n$ by 5.4.2. By 5.5.4 and 5.5.5, it follows that either $w^{\mathbb{Z}} x \subset H(\alpha)$ or $w^{\mathbb{Z}} x \subset \mu(\alpha)$. In the latter case, $\mu\left(w^{n} \alpha\right)$ passes through $x$ for all $n \in \mathbb{Z}$. This implies that $\alpha$ is periodic, a contradiction. This proves that $Q(w) \subset H(\alpha)$.

5.5.9 Definition. Call a root $\alpha w$-supporting if $Q(w) \subset H(\alpha)$. Similarly for halfspaces.

The above lemma says that for every even root $\alpha$, one of $\alpha,-\alpha$ is supporting. From the results of the following section it will follow that the other one is not supporting.

5.6. Non-emptiness of the axis. In this section we will prove that $Q(w)$ is nonempty. It will be useful to consider subsets $Q_{1}(w) \subset Q_{2}(w) \subset Q(w)$ as follows.

5.6.1 Definition. Define $Q_{2}(w)$ to be the set of $x \in Q(w)$ such that for all outward $\alpha,\langle x, \alpha\rangle \leq\langle x, w \alpha\rangle$, and for all periodic $\alpha,\langle x, \alpha\rangle=\langle x, w \alpha\rangle$. Let $Q_{1}(w)=$ $Q_{2}(w) \cap E_{>0}$, where $E_{>0}$ denotes the sum of the generalized eigenspaces in $E^{*}$ of $w$ with eigenvalue in $\mathbb{R}_{>0}$.

The set $Q_{1}(w)$ will be used in Section 6.5. It is left to the reader to check the analogues to 5.5.3.

Whereas $Q(w)$ is a purely combinatorial object (that is, it is a union of facets), $Q_{1}(w)$ and $Q_{2}(w)$ are not. On the other hand, we are able to prove that $Q_{1}(w)$ and $Q_{2}(w)$ are convex, which is only conjectural for $Q(w)$ (see 5.7.10).

5.6.2 Proposition. $Q_{1}(w)$ and $Q_{2}(w)$ are convex.

Proof. It is enough to prove that $Q_{2}(w)$ is convex, for it immediately follows that $Q_{1}(w)$ is convex too. Let $x, y \in Q_{2}(w)$, and write $z=x+y$. It is enough to show that $z \in Q(w)$, for it would then easily follow that $z \in Q_{2}(w)$. For every root $\alpha$, we must prove that $n \mapsto f_{\alpha}\left(w^{n} z\right)$ is monotonic (5.5.4). For $\alpha$ even this follows from 5.5.8. For $\alpha$ periodic, $n \mapsto\left\langle z, w^{n} \alpha\right\rangle$ is constant zero by definition of $Q_{2}(w)$, whence so is $n \mapsto f_{\alpha}\left(w^{n} z\right)$. For $\alpha$ odd, $n \mapsto\left\langle z, w^{n} \alpha\right\rangle$ is monotonic by definition of $Q_{2}(w)$, whence so is $n \mapsto f_{\alpha}\left(w^{n} z\right)$.

5.6.3 Lemma. There exists a constant $N=N(W)$ such that for all $w \in W$ and every $w$-periodic root $\alpha$, we have $w^{N} \alpha=\alpha$. 
Proof. We may suppose that $E$ is the classical root base. Let $K$ be the number field generated by $\left\{\cos \left(\pi / m_{s t}\right) \mid s, t \in S\right\}$. Let $E_{K} \subset E$ be the $K$-vector space with basis $\left\{e_{s} \mid s \in S\right\}$. Note that $\Phi \subset E_{K}$. Let $w \in W$, and let $\alpha \in \Phi$ be $w$-periodic. Let $V \subset E_{K}$ be the $\mathbb{Q}$-vector space spanned by $w^{\mathbb{Z}} \alpha$. There exists a $w$-invariant decomposition of $\mathbb{Q}$-vector spaces $V=\bigoplus V_{i}$ and natural numbers $n_{i}$ such that the characteristic polynomial of $\left.w\right|_{V_{i}}$ is the $n_{i}$-th cyclotomic polynomial. In particular, $\phi\left(n_{i}\right)=\operatorname{dim} V_{i} \leq \operatorname{dim} V \leq \operatorname{dim}_{\mathbb{Q}} E_{K}$. Since $\lim _{n \rightarrow \infty} \phi(n)=\infty$, the $n_{i}$ are bounded by a constant $m$ depending only on $W$. Putting $N=\operatorname{lcm}(1,2, \ldots, m)$, we have $w^{N} \alpha=\alpha$, which proves the theorem.

5.6.4 Remark. A better bound for the constant $N$ of 5.6 .3 will be given in 6.3.12. The proof of this result will depend indirectly on 5.6.3.

5.6.5 Theorem. There exists a constant $N=N(W)$ with $\left(1+w+w^{2}+\cdots+\right.$ $\left.w^{N-1}\right) U^{0} \subset Q_{2}(w)$ for all $w \in W$.

Proof. Let $N_{0}$ be the constant defined in 5.3.2, so that for every root $\alpha$, there exists $k$ with $1 \leq k \leq N_{0}$ and $\left|\left(\alpha, w^{k} \alpha\right)\right| \geq 1$. Let $N_{1}$ be the constant of 5.6.3. Let $N_{2}=2 \operatorname{lcm}\left(1,2, \ldots, N_{0}\right), N=\operatorname{lcm}\left(N_{1}, N_{2}\right)$. We will show that $N$ has the desired property. Let $x \in U^{0}$. Let $y=\left(1+w+\cdots+w^{N-1}\right) x$. Note that $w y-y=w^{N} x-x$. By 5.5.4 and 5.5.5, it is enough to show that $\langle y, \alpha\rangle=\langle w y, \alpha\rangle$ for every periodic $\alpha$, and $\langle w y, \alpha\rangle \leq\langle y, \alpha\rangle$ for every outward $\alpha$, and $\left\langle w^{\mathbb{Z}} y, \alpha\right\rangle \subset \mathbb{R}_{>0}$ or $\mathbb{R}_{<0}$ for every even $\alpha$.

Let $\alpha$ be a periodic root. By our choice of $N_{1}, w^{N} \alpha=\alpha$. Hence $\langle w y-y, \alpha\rangle=$ $\left\langle w^{N} x-x, \alpha\right\rangle=0$.

Next let $\alpha$ be outward. By 5.3.2 and 1.4.7, there exists $k, 1 \leq k \leq N_{0}$, with $\alpha<w^{k} \alpha$. By 1.4.7(d), it follows that $\left\langle w^{n+k} x, \alpha\right\rangle \leq\left\langle w^{n} x, \alpha\right\rangle$ for all $n \in \mathbb{Z}$. Since $k \mid N$, we find $\left\langle w^{N} x, \alpha\right\rangle \leq\langle x, \alpha\rangle$. Hence $\langle w y-y, \alpha\rangle=\left\langle w^{N} x-x, \alpha\right\rangle \leq 0$.

Finally, consider the case of even $\alpha$. By 5.3.2, there exists $k$ with $1 \leq k \leq N_{0}$ and $\left(\alpha, w^{k} \alpha\right) \leq-1$. By 1.4.7, the set $\left\langle U^{0}, \alpha+w^{k} \alpha\right\rangle$ is contained in $\mathbb{R}_{>0}$ or $\mathbb{R}_{<0}$, say in $\mathbb{R}_{>0}$. We will show that $\langle y, \alpha\rangle>0$, whence $\left\langle w^{\mathbb{Z}} y, \alpha\right\rangle \subset \mathbb{R}_{>0}$, since the same argument applies to $w^{p} y$ too. Since $2 k$ divides $N$, we can partition the set $\{0,1, \ldots, N-1\}$ into pairs, each having difference $k$. For each pair, say $\{n, n+k\}$, we have $\left\langle w^{n} x+w^{n+k} x, \alpha\right\rangle>0$. Adding over all pairs, we find that $\langle y, \alpha\rangle=$ $\left\langle\left(1+w+\cdots+w^{N-1}\right) x, \alpha\right\rangle>0$.

5.6.6 Corollary. For every $w \in W$, the limit $\lim _{n \rightarrow \infty} \ell\left(w^{n}\right) / n$ exists and equals $r(w)$.

Proof. By 5.6.5, $Q(w)$ is non-empty. Let $x \in Q(w)$. Then for all $n \in \mathbb{N}$, we have, by 5.4 .1 and definition of $Q(w)$,

$$
\left|\ell\left(w^{n}\right)-n d_{\Phi}(x, w x)\right|=\left|d_{\Phi}\left(C, w^{n} C\right)-d_{\Phi}\left(x, w^{n} x\right)\right| \leq 2 d_{\Phi}(C, x),
$$


which shows that $\lim _{n \rightarrow \infty} \ell\left(w^{n}\right) / n$ exists and equals $d_{\Phi}(x, w x)=r(w)$.

5.6.7 Corollary. We have $d_{\Phi}(C, Q(w))=\mathcal{O}(\ell(w))$ for all $w \in W$. More precisely, $d_{\Phi}(C, Q(w)) \leq\left(\begin{array}{c}N \\ 2\end{array}\right) \ell(w)$, where $N$ is the constant of 5.6.5.

Proof. Let $x \in C$ and put $y=\left(1+w+\cdots+w^{N-1}\right) x$. Then $y \in Q(w)$ by 5.6.5. Furthermore, each root separating $C$ from $y$ separates $C$ from one of $x, w x, \ldots, w^{N-1} x$, and similarly for strict separation. Hence

$$
d_{\Phi}(C, y) \leq \sum_{k=0}^{N-1} d_{\Phi}\left(x, w^{k} x\right)=\sum_{k=0}^{N-1} \ell\left(w^{k}\right) \leq \sum_{k=0}^{N-1} k \ell(w)=\left(\begin{array}{c}
N \\
2
\end{array}\right) \ell(w) .
$$

Let $P[X]$ denote the set of non-zero real polynomials in $X$ with non-negative coefficients, $P[X]=\left\{a_{0}+a_{1} X+\cdots+a_{n} X^{n} \in \mathbb{R}[X]-\{0\} \mid a_{i} \geq 0\right.$ for all $\left.i\right\}$.

5.6.8 Lemma. Let $z$ be a complex number. Then there exists $f \in P[X]$ with $f(z)=0$ if and only if $z$ is not a positive real number.

Proof. 'Only if' is easy. To prove 'if', note that there exists $n \in \mathbb{Z}_{>0}$ such that $\operatorname{Re}\left(z^{n}\right) \leq 0$. Now $f=\left(X^{n}-z^{n}\right)\left(X^{n}-\bar{z}^{n}\right) \in P[X]$ satisfies $f(z)=0$.

5.6.9 Lemma. Let $A \in \mathrm{GL}_{n}(\mathbb{R})$. Then there exists $f \in P[X]$ such that all eigenvalues of $f(A)$ are in $\mathbb{R}_{\geq 0}$.

Proof. Let $f_{0} f_{1} \in \mathbb{R}[X]$ be the characteristic polynomial of $A$, and assume $f_{0}(\lambda)=$ $0 \Longrightarrow \lambda \in \mathbb{R}_{>0}$ and $f_{1}(\lambda)=0 \Longrightarrow \lambda \notin \mathbb{R}_{>0}$ and that $f_{0}$ is monic. By 5.6.8, there exists $f \in P[X]$ such that $f_{1}$ divides $f$. It is easy to see that $f$ has the desired property.

5.6.10 Theorem. For every $w \in W$, the set $Q_{1}(w)$ is non-empty.

Proof. By 5.6.9, there exists $f \in P[X]$ such that all eigenvalues of $f(w)$ are in $\mathbb{R}_{\geq 0}$. By 5.6.5, $Q_{2}(w)$ is non-empty; let $y \in Q_{2}(w)$. Let $z=f(w) y$. Then $z \in Q_{2}(w)$ since $Q_{2}(w)$ is convex. Moreover, $z \in E_{>0}$ by our construction of $f$. Hence $z \in E_{>0} \cap Q_{2}(w)=Q_{1}(w)$.

5.7. Critical roots. In this section we fix an element $w \in W$.

5.7.1 Lemma. Let $x \in U^{0}, N>0$. Then there are only finitely many roots $\alpha$ with $0 \leq\langle x, \alpha\rangle \leq N$. 
Proof. We may suppose that $E=\operatorname{Span}(\Pi)$. First we prove the assertion for the special case of $x$ being on no wall. After translation by an element of $W$, we may suppose that $x \in C$. Since $C$ is open, there exists a basis $\left\{x_{1}, \ldots, x_{r}\right\}$ of $E$ such that $x_{i} \in C$ for all $i$, and $x=x_{1}+\cdots+x_{r}$. Write

$$
X=\{\alpha \in \Phi \mid 0 \leq\langle x, \alpha\rangle \leq N\} .
$$

Note that $X \subset \Phi^{+}$. Thus $X$ is a subset of the compact set $\left\{y \in E \mid\left\langle x_{i}, y\right\rangle \geq\right.$ 0 for all $\left.i, \sum_{i}\left\langle x_{i}, y\right\rangle \leq N\right\}$. Moreover, $X$ is a discrete set by 1.2.5. We conclude that $X$ is finite, which proves the case $x$ on no wall.

Now we will solve the general case. There exist $y, z \in U^{0}$ on no wall such that $x=y+z$. Now except from the finitely many roots $\alpha$ with $d_{\alpha}(y, z)>0$, the inequality $0 \leq\langle x, \alpha\rangle$ implies that $0 \leq\langle y, \alpha\rangle,\langle z, \alpha\rangle$, whence

$$
\langle x, \alpha\rangle \in[0, N] \Longrightarrow\langle y, \alpha\rangle,\langle z, \alpha\rangle \in[0, N] .
$$

Thus, the general case follows from the special one by applying it to $y$.

5.7.2 Theorem. Let $\alpha \in \Phi$, and let $A \subset W$ be the corresponding half-space. Then the following assertions are equivalent.

(1) Span $w^{\mathbb{Z}} \alpha$ is positive definite and $\sum_{n=1}^{k} w^{n} \alpha=0$, where $k$ is the smallest positive integer with $w^{k} \alpha=\alpha$.

(2) $\bigcap_{n \in \mathbb{Z}} w^{n} A=\bigcap_{n \in \mathbb{Z}} w^{n}(W-A)=\varnothing$, and $A$ is periodic.

(3) $Q(w) \subset \mu(\alpha)$.

(4) $Q_{2}(w) \subset \mu(\alpha)$.

(5) $Q_{1}(w) \subset \mu(\alpha)$.

As to (1), note that if $\operatorname{Span} w^{\mathbb{Z}} \alpha$ is positive definite, then $\alpha$ is periodic by 1.2.6.

Proof. (1) $\Longrightarrow$ (2) Suppose that $\bigcap_{n \in \mathbb{Z}} w^{n} A \neq \varnothing$. Then there exists $x \in U^{0}$ such that $\left\langle x, w^{n} \alpha\right\rangle>0$ for all $n \in \mathbb{Z}$. Hence $0=\langle x, 0\rangle=\sum_{n=1}^{k}\left\langle x, w^{n} \alpha\right\rangle>0$, a contradiction. Hence $\bigcap_{n \in \mathbb{Z}} w^{n} A=\varnothing$ and similarly $\bigcap_{n \in \mathbb{Z}} w^{n}(W-A)=\varnothing$. Clearly, $A$ is periodic.

(2) $\Longrightarrow$ (3) We will prove that for periodic $A, \bigcap_{n \in \mathbb{Z}} w^{n} A=\varnothing$ implies $Q(w) \subset$ $K(W-A)$. Consequently, (2) implies $Q(w) \subset K(A) \cap K(W-A)=\mu(A)$. Let $x \in Q(w) \cap H(A)$. Let $k>0$ be such that $w^{k} A=A$. Then $w^{k \mathbb{Z}} x \subset H(A)$. By 5.5.4, we find $w^{\mathbb{Z}} x \subset H(A)$. Let $g \in W$ be such that $x \in g \bar{C}$. It follows that $g \in \bigcap_{n \in \mathbb{Z}} w^{n} A$, a contradiction.

(3) $\Longrightarrow(4) \Longrightarrow$ (5) is trivial.

$(5) \Longrightarrow(1)$ By 5.6.10, $Q_{1}(w)$ is non-empty; choose any $x \in Q_{1}(w)$. Since $Q_{1}(w)$ is $w$-invariant, (5) gives $w^{\mathbb{Z}} x \subset \mu(\alpha)$, whence $x \in \bigcap_{n \in \mathbb{Z}} w^{n} \mu(\alpha)$. Since $x \in U^{0}$, it follows that $P:=\operatorname{Span} w^{\mathbb{Z}} \alpha$ is positive definite (for $P$ is contained in the span 
of the root system $\{\beta \in \Phi \mid\langle x, \beta\rangle=0\}$, which is finite by 2.2.5). In particular, as noted above, $\alpha$ is periodic, say $w^{k} \alpha=\alpha, k>0$ minimal. Let $u=\sum_{n=1}^{k} w^{n} \alpha$ and let $u^{*}=(u, \cdot) \in E^{*}$. Consider $y=x+\varepsilon u^{*}$, where $\varepsilon>0$ will be chosen later on.

First note that, by $1.2 .2(\mathrm{~d})$, there exists $\eta>0$ such that if $0<\varepsilon<\eta$ then $y \in U^{0}$, and for all $\beta \in \Phi: d_{\beta}(x, y)>0 \Longrightarrow x \in \mu(\beta)$. By 5.7.1, there exists $\delta>0$ such that for all $\beta \in \Phi$, either $\langle x, \beta\rangle=0$ or $|\langle x, \beta\rangle| \geq \delta$. Let $N>\max \left\{\left|\left\langle u^{*}, \beta\right\rangle\right| \mid \beta \in\right.$ $\Phi, x \in \mu(\beta)\}$ (note that this is possible since there exist only finitely many $\beta \in \Phi$ with $x \in \mu(\beta))$. Choose $\varepsilon$ such that $0<\varepsilon<\min (\eta, \delta / N)$.

We will show that $y \in Q_{1}(w)$. It is enough to show that $y \in Q(w)$, because the additional properties for $Q_{1}(w)$ follow from the fact that $x \in Q_{1}(w)$ and $w u^{*}=u^{*}$. We must show that for all $\beta \in \Phi$, the function $\mathbb{Z} \rightarrow\{-1 / 2,0,1 / 2\}, n \mapsto f_{\beta}\left(w^{n} y\right)$ is monotonic - see 5.5.4.

If $d_{\beta}\left(w^{n} x, w^{n} y\right)=0$ for all $n$, there is nothing to prove. Otherwise, we may translate $\beta$ over a power of $w$ so as to have $d_{\beta}(x, y)>0$ (and hence $x \in \mu(\beta)$ and $\left|\left\langle u^{*}, \beta\right\rangle\right|<N$ by the above). Since $w u^{*}=u^{*}$, we have for all $n \in \mathbb{Z}$ :

$$
\left\langle w^{n} y, \beta\right\rangle=\left\langle w^{n}\left(x+\varepsilon u^{*}\right), \beta\right\rangle=\left\langle w^{n} x, \beta\right\rangle+\varepsilon\left\langle u^{*}, \beta\right\rangle .
$$

The sign of $\left\langle w^{n} y, \beta\right\rangle$ is determined by the first of the two terms on the right. More precisely, if $\left\langle w^{n} x, \beta\right\rangle \neq 0$ then

$$
\left|\varepsilon\left\langle u^{*}, \beta\right\rangle\right| \leq\left|\frac{\delta}{N}\left\langle u^{*}, \beta\right\rangle\right| \leq \delta<\left|\left\langle w^{n} x, \beta\right\rangle\right| .
$$

In other words, $f_{\beta}\left(w^{n} x\right) \neq 0 \Longrightarrow f_{\beta}\left(w^{n} y\right)=f_{\beta}\left(w^{n} x\right)$. Furthermore, if on the contrary $f_{\beta}\left(w^{n} x\right)=0$, then $f_{\beta}\left(w^{n} y\right)$ has the same sign as $\left\langle u^{*}, \beta\right\rangle$, that is, a constant independent of $n$. It follows that $n \mapsto f_{\beta}\left(w^{n} y\right)$ is monotonic. Hence $y \in Q_{1}(w)$.

Since $x, y \in Q_{1}(w) \subset \mu(\alpha)$, we have $\langle x, \alpha\rangle=\langle y, \alpha\rangle=0$. It follows that $\left\langle u^{*}, \alpha\right\rangle=0$ or, equivalently, $(u, \alpha)=0$. Since $w u=u$ and $P=\operatorname{Span} w^{\mathbb{Z}} \alpha$, we have $u \perp P$. Since $u \in P$ and $P$ is non-degenerate, we have $u=0$. This proves (1).

5.7.3 Definition. A half-space, root or reflection is called $w$-critical if it satisfies the equivalent conditions of 5.7.2. It is called $w$-essential if it is either $w$-critical or $w$-odd.

5.7.4 Examples. (a) Let $W$ be finite. After conjugation, we may write $\operatorname{Pc}(w)=W_{I}$. Now $Q(w)=K_{I}$ and the set of critical roots equals $\Phi_{I}$.

(b) Let $w$ be torsion. Then $\operatorname{Pc}(w)$ is a finite parabolic subgroup. After conjugating $w$, we may write $\operatorname{Pc}(w)=W_{I}$. Now $Q(w)=\left\{x \in U^{0} \mid d_{\Phi}(x, w x)=0\right\}=K_{I}$. Moreover, $\alpha$ is critical if and only if $Q(w) \subset \mu(\alpha)$, that is, $r_{\alpha} \in\left(K_{I}\right)^{\prime}$. Since $I$ is spherical, it is facial by 2.2.4. By 2.1.4, $\left(K_{I}\right)^{\prime}=W_{I}$. Thus, the set of critical roots equals $\Phi_{I}$. 
(c) Let us go back to example (c) of 5.2.5. Using criterion 5.7.2 (2), we find that the only critical wall is the thick line. In 5.5.2 (c), we remarked that $Q(w)$ equals the thick line. The reader is invited to check that conditions 5.7.2(1)-(3) are equivalent for all roots.

An overview of our classification of roots is given in Figure 8. A periodic root $\alpha$ is

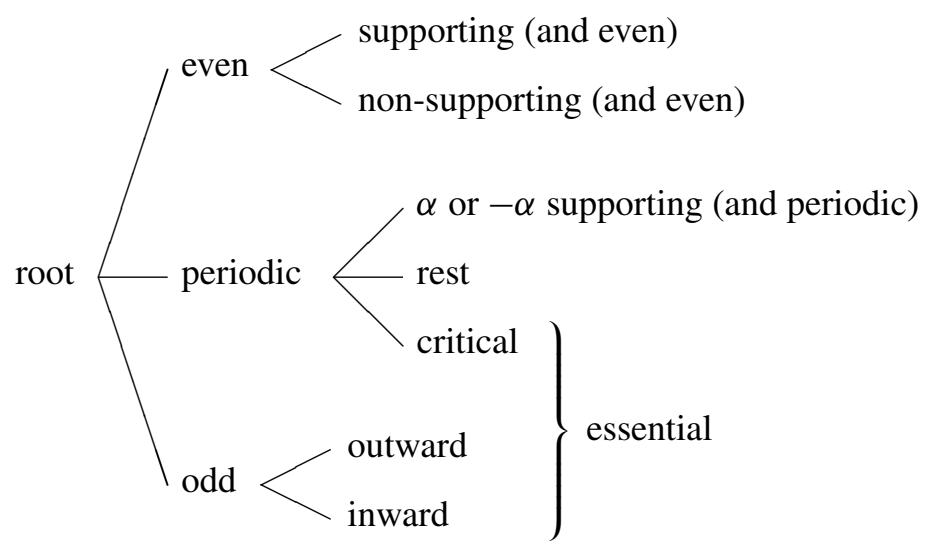

Figure 8. Classes of roots.

called $w$-rest if it is not critical and $\alpha$ and $-\alpha$ are not supporting. An example of a $w$ rest root is every root, if $w=1$. Examples of supporting periodic roots can be found in example (c) of 5.2.5. It is easily seen that to each horizontal line different from the thick one, one of the associated roots is supporting periodic. Another nice example is that (in the same example) every $w$-periodic root is $w^{2}$-rest (since $Q\left(w^{2}\right)=U^{0}$ as $w^{2}$ is a translation - see 5.5.2(d)).

5.7.5 Proposition. Let $A \subset W$ be a half-space. Then the following assertions are equivalent.

(1) A is essential.

(2) $\bigcap_{n \in \mathbb{Z}} w^{n} A=\bigcap_{n \in \mathbb{Z}} w^{n}(W-A)=\varnothing$.

Proof. (1) $\Longrightarrow$ (2) follows directly from the definitions of critical and odd.

(2) $\Longrightarrow$ (1) By condition 5.7.2(2) we need only show that $A$ is not even. Suppose it is, say supporting. Let $x \in Q(w)$ and $g \in W$ be such that $x \in g \bar{C}$. Now from $w^{\mathbb{Z}} x \subset Q(w) \subset H(A)$ we find $w^{\mathbb{Z}} g \subset A$, contradicting (2).

5.7.6 Proposition. The set of critical roots is a finite, parabolic root subsystem of $\Phi$. 
Proof. This follows immediately from criterion 5.7.2 (3), 2.1.4 together with the fact that $\varnothing \neq Q(w) \subset U^{0}$.

We know that for an even $\alpha$, either $\alpha$ or $-\alpha$ is supporting, and odd roots are never supporting. The following proposition characterizes supporting periodic roots.

5.7.7 Proposition. Let $\alpha$ be periodic. Then the following assertions are equivalent.

(1) $Q(w) \cap \mu(\alpha) \neq \varnothing$.

(2) Span $w^{\mathbb{Z}} \alpha$ is positive definite.

(3) Neither $\alpha$ nor $-\alpha$ is supporting.

Proof. Let $f \in P[X]$ be such that $f(w) U^{0} \subset Q_{2}(w)$ (see 5.6.5).

(1) $\Longrightarrow(2)$ Let $x \in Q(w) \cap \mu(\alpha)$. Let $k>0$ be such that $w^{k} \alpha=\alpha$. Then $w^{k \mathbb{Z}} x \subset \mu(\alpha)$. Since $x \in Q(w)$, this implies $w^{\mathbb{Z}} x \subset \mu(\alpha)$ by 5.5.4. Hence $\operatorname{Span} w^{\mathbb{Z}} \alpha$ is positive definite by 2.2.5 and 1.2.6.

(2) $\Longrightarrow$ (3) By 3.2.3, there exists $x \in U^{0}$ such that $x \in w^{n} \mu(\alpha)$ for all $n \in \mathbb{Z}$. Now $f(w) x \in Q_{2}(w) \cap \mu(\alpha)$, whence (3).

(3) $\Longrightarrow$ (1) Suppose that (3) holds but not (1). Then there are $x \in Q(w) \cap H(\alpha)$ and $y \in Q(w) \cap H(-\alpha)$. Since $x \in Q(w)$ and $\alpha$ is not odd, we have $w^{\mathbb{Z}} x \subset H(\alpha)$ by 5.5.5. Hence $f(w) x \in Q_{2}(w) \cap H(\alpha)$, and similarly $f(w) y \in Q_{2}(w) \cap H(-\alpha)$. Let $t>0$ be such that $f(w)(x+t y) \in \mu(\alpha)$. Since $Q_{2}(w)$ is convex, we have $f(w)(x+t y) \in Q_{2}(w) \cap \mu(\alpha) \subset Q(w) \cap \mu(\alpha)$.

We finish the section with a conjecture on $Q(w)$.

5.7.8 Definition. Define $Q^{*}(x)$ to be the intersection of all $H(\alpha)$ containing $Q(w)$, and all $\mu(\alpha)$ containing $Q(w)$.

5.7.9 Conjecture. $Q(w)=Q^{*}(w)$.

The reader is invited to check this conjecture for the examples of 5.5.2 (e). Conjecture 5.7.9 does not seem to be more difficult than its special case where there are no periodic roots at all.

Conjecture 5.7.9 easily implies the following statements. None of these statements however has been proved yet without use of 5.7.9. We note that 5.7.10(d) can be reformulated in a purely combinatorial way.

5.7.10 Conjecture. (a) $Q(w)$ is combinatorially convex, that is, it is the intersection of a family of (closed or open) half-spaces in $U^{0}$.

(b) $Q(w)$ is open in the intersection of those $\mu(\alpha)$ containing $Q(w)$.

(c) $Q(w)$ is convex.

(d) $Q(w)$ is topologically connected. 
5.8. The parabolic closure. For this section fix $w \in W$.

Let us call a point $x \in Q(w)$ general if for all roots $\alpha$, the condition $x \in \mu(\alpha)$ implies that $Q(w) \subset \mu(\alpha)$, that is, $\alpha$ is critical.

5.8.1 Theorem. Consider the following subgroups of $W$ :

$H=$ the parabolic closure of $w$,

$K=$ the standard parabolic closure of $w$,

$L=$ the subgroup generated by the essential reflections.

If there exists general $x \in Q(w)$ such that $x \in \bar{C}$, then $H=K=L$.

Proof. $H \subset K$ follows from the fact that every standard parabolic subgroup is a parabolic subgroup.

$K \subset L$. It is enough to show that every root separating 1 from $w$ is essential. Let $\alpha$ be a root separating 1 from $w$. We have

$$
0<d_{\alpha}(C, w C) \leq d_{\alpha}(C, x)+d_{\alpha}(x, w x)+d_{\alpha}(w x, w C) .
$$

Thus, one of the terms on the right is positive. If $d_{\alpha}(x, w x)>0$, it follows that $\alpha$ is odd by 5.5.5. If $d_{\alpha}(C, x)>0$ then $x \in \mu(\alpha)$, whence $\alpha$ is critical. Similarly if $d_{\alpha}(w x, w C)>0$.

$L \subset H$. Note that $H$ and $L$, contrary to $K$, are conjugacy covariant, that is, $\operatorname{Pc}\left(g w g^{-1}\right)=g \operatorname{Pc}(w) g^{-1}$ and similarly for $L$. By conjugating $w$, we may suppose $H$ to be standard parabolic. Note that we then no longer may assume $x \in \bar{C} \cap Q(w)$, but we do not need this. Let $A$ be an essential half-space. Then by 5.7.5, $w^{\mathbb{Z}} \not \subset A$ and $w^{\mathbb{Z}} \not \subset W-A$. Hence $H \not \subset A$ and $H \not \subset W-A$. Let $p \in H \cap A, q \in H \cap(W-A)$. Since $H$ is standard parabolic (that is, connected in the Cayley graph), there exists a path in $H$ (viewed as subgraph of the Cayley graph) from $p$ to $q$. One of the edges of this path is from $A$ to $W-A$, say $\{y, y s\}$. It follows that $r_{A}=y s y^{-1} \in H$, which proves $L \subset H$.

We note that in 5.8.1, instead of $x$ being general, it is sufficient that $x$ lies on only essential walls. The proof is the same.

5.8.2 Lemma. $Q(w)$ contains general points.

Proof. By 5.6.2, $Q_{2}(w)$ is a non-empty cone. By 2.1.2, there exists $x \in Q_{2}(w)$ such that $x^{\prime}=Q_{2}(w)^{\prime}$. Now for every root $\alpha$, if $x \in \mu(\alpha)$ then $Q_{2}(w) \subset \mu(\alpha)$, whence $\alpha$ is critical by 5.7.2. Furthermore, $x \in Q_{2}(w) \subset Q(w)$. Hence $x$ is a general point in $Q(w)$.

5.8.3 Theorem. The parabolic closure of $w$ equals the subgroup of $W$ generated by the $w$-essential reflections. 
Proof. By 5.8.2, there exists a general point $x \in Q(w)$. By conjugating $w$, we may suppose that $x \in \bar{C}$. The result now follows from 5.8.1.

Subsequently, Theorem 5.8.3 was used in [44].

5.8.4 Definition. Denote the root system generated by the odd roots by $\Phi_{\text {odd }}$.

5.8.5 Lemma. Let $\alpha$ be periodic. Then either $\alpha \perp \Phi_{\text {odd }}$ or $\alpha \in \Phi_{\text {odd }}$.

Proof. Suppose that $\alpha$ is not perpendicular to $\Phi_{\text {odd }}$, say $(\alpha, \beta) \neq 0, \beta$ odd. We may suppose $\beta$ to be outward, and $(\alpha, \beta)<0$. Let $\gamma=r_{\beta} \alpha=\alpha-2(\alpha, \beta) \beta$. We will show that $\gamma$ is odd. Let $x \in U^{0}$ be any point. By 5.7.1, we have

$$
\lim _{n \rightarrow \infty}\left\langle x, w^{n} \beta\right\rangle=\infty, \quad \lim _{n \rightarrow-\infty}\left\langle x, w^{n} \beta\right\rangle=-\infty .
$$

Hence

$$
\left\langle x, w^{n} \gamma\right\rangle=\left\langle x, w^{n} \alpha-2(\alpha, \beta) w^{n} \beta\right\rangle \rightarrow \infty, \quad n \rightarrow \infty,
$$

and similarly $\left\langle x, w^{n} \gamma\right\rangle \rightarrow-\infty$ as $n \rightarrow-\infty$. Thus, $\gamma$ is odd. Hence $\alpha=r_{\beta} \gamma \in \Phi_{\text {odd }}$.

5.8.6 Definition. We define $\mathrm{Pc}^{\infty}(w)$ to be the group generated by the $w$-odd reflections, and $\mathrm{Pc}^{0}(w)$ to be the group generated by $r_{\alpha}$ for all critical roots perpendicular to $\Phi_{\text {odd }}$.

5.8.7 Corollary. We have $\operatorname{Pc}(w)=\mathrm{Pc}^{\infty}(w) \times \mathrm{Pc}^{0}(w)$. Furthermore, $\mathrm{Pc}^{\infty}(w)$ is the product of the infinite components of $\operatorname{Pc}(w)$.

Proof. The first statement follows immediately from 5.8.5 and 5.8.3. As to the second statement, for every component $H \subset \operatorname{Pc}(w)$, at least one of the following is true.

(1) $H$ is generated by the odd reflections in $H$.

(2) $H$ is generated by the critical reflections in $H$.

In case (1) holds, $H$ is infinite, since for every odd reflection $r \in H$, all $w^{n} r w^{-n}$ are different $(n \in \mathbb{Z}$ ). In case (2), $H$ is finite by 5.7.6. (In particular, (1) and (2) cannot both hold.)

5.8.8 Example. An example of a critical root $\alpha \in \Phi_{\text {odd }}$ is the (up to sign unique) critical root in the Coxeter group of 5.2.5 (c). Namely, $\Phi_{\text {odd }}=\Phi$.

Examples of critical roots $\alpha \perp \Phi_{\text {odd }}$ are easily constructed, for example with $w$ torsion, that is, $\Phi_{\text {odd }}=\varnothing$. 
The preceding results do not imply a fast and easy to implement algorithm computing $\operatorname{Pc}(w)$ for given $w$. To this end, we designed the following funny variation of 5.8.1. Namely, we replace $Q(w)$ by $Q^{*}(w)$, which is conjecturally the same set by 5.7.9. Theorem 5.8.1 is an ingredient to the proof of 5.8.9. Again, call a point in $Q^{*}(w)$ general if it lies on only critical walls.

5.8.9 Corollary. Let $x \in Q^{*}(w)$ be a general point, and assume that $x \in \bar{C}$. Then $\operatorname{Pc}(w)$ is a standard parabolic subgroup of $W$.

Proof. Let $I \subset S$ be such that $x \in C_{I}$. Since $x$ is general, the critical reflections generate the standard parabolic group $W_{I}$. The proof will be finished by showing that $\mathrm{Pc}^{\infty}(w)$ is a standard parabolic subgroup too. By 5.6.3 and 5.3.2, there exists $n>0$ such that all $w$-periodic roots are $w^{n}$-invariant, and for every outward root $\alpha$, we have $\alpha<w^{n} \alpha$. For every supporting even root $\alpha$, we have $x \in Q^{*}(w) \subset H(\alpha)$ and hence $C \subset H(\alpha)$. Now it is easy to see that $C \subset Q\left(w^{n}\right)$. By 5.8.1, $\operatorname{Pc}\left(w^{n}\right)$ is a standard parabolic subgroup. Since all periodic roots are $w^{n}$-invariant, there are no $w^{n}$-critical roots - use, for example, criterion 5.7.2(1). Hence $\operatorname{Pc}\left(w^{n}\right)=\operatorname{Pc}^{\infty}\left(w^{n}\right)=\operatorname{Pc}^{\infty}(w)$ by 5.2 .7 .

5.8.10 Remark. The above result can be strengthened by replacing $Q^{*}(w)$ by $Q^{+}(w)$, which is defined to be the intersection of $H(\alpha)$ for all supporting even $\alpha$, and $\mu(\alpha)$ for all critical $\alpha$. The difference with $Q^{*}(w)$ is that supporting periodic roots are removed. The proof is the same. It is an open question whether $Q^{+}(w)=Q^{*}(w)$. It is true for the examples of 5.5.2 (e).

5.9. Calculations in number fields. For the subsequent section with algorithms on Coxeter groups we need some facts on algorithms for calculations in number fields and their complexity. These algorithms are guaranteed to give the correct answers. They compute with algebraic numbers without rounding. Therefore the algorithms cannot be compared to numerical algorithms doing similar things.

Let $K$ be a number field, that is, a finite extension of $\mathbb{Q}$. Given a $\mathbb{Q}$-basis $x_{i}$ of $K$, we define the height on $K$ by

$$
h\left(\sum_{i} a_{i} x_{i} / a\right)=\log \left(\sum_{i} a_{i}^{2}+a^{2}\right),
$$

where $a_{i}$ and $a$ are relatively prime integers. The height of a polynomial (or rational function, or matrix) is by definition the maximum of the heights of its coefficients (or entries).

5.9.1. Calculations in number fields. Let $x, y \in K$. Then $x+y$ can be computed in $\mathcal{O}(h(x)+h(y))$ time, whereas $x y$ and $x / y$ can be computed in $\mathcal{O}(h(x) h(y))$ time. There exists a faster algorithm with complexity $\mathcal{O}\left((h(x)+h(y))^{1+\varepsilon}\right)$ for every $\varepsilon>0$, using Fourier transforms [39], p. 278. We will not consider this improvement of the bounds of complexity. 
5.9.2. Signs of real algebraic numbers. Fix a real number field $K$. Then there exists an algorithm that, given $f, g \in K[X]$ and an integer $i$, decides whether $g(a)<0$ or $g(a)=0$ or $g(a)>0$, where $a$ is the $i$-th real root of $f$ in the natural ordering (supposing $a$ exists). Its complexity is $\mathcal{O}\left(n^{6} h^{2}\right)$, where $n$ is a bound for the degrees of $f, g$, and $h$ a bound for their heights [21], p. 93. See also [29], [24], Section 3.2.1.1. It is easy to deduce from Tarski's generalization of Sturm's theorem [38], VI.10, that for fixed $n$, the complexity is $\mathcal{O}\left(h^{2}\right)$. This suffices for us, since we are only interested in the case where $n$ is bounded (because we fix one Coxeter group). A particular case is that for all $x \in K$, it can be decided in $\mathcal{O}\left(h(x)^{2}\right)$ time whether $x>0$.

5.10. Algorithms. In this section we give a chain of algorithms, leading to a computation of the parabolic closure. The algorithms make extensive use of the representation and the Tits cone.

Fix a root basis $(E,(\cdot, \cdot), \Pi)$ defined over a real number field $K-$ see 4.7.5. For each of the algorithms, part of the input is $w \in W$, given by a string. The first step in every algorithm is the computation of the matrix of $w$ and, in some cases, the roots separating 1 from $w$. By 5.9.1, this can be done in $\mathcal{O}\left(\ell(w)^{2}\right)$ time. The height $h(w)$ is $\mathcal{O}(\ell(w))$.

Following [10], define the depth of a root $\alpha$ to be

$$
\mathrm{dp}(\alpha)=\frac{\ell\left(r_{\alpha}\right)+1}{2} .
$$

We have $h(\alpha)=\mathcal{O}(\operatorname{dp}(\alpha))$. For a vector $x \in U^{0}$, defined over a (fixed) number field, the quantity $\max \left\{h(x), d_{\Phi}(x, C)\right\}$ will also be called depth and denoted by $\operatorname{dp}(x)-$ we are running out of names and the two notions of depth will be used for the same purpose.

An algorithm is called quadratic, cubic, ... if has complexity $\mathcal{O}\left(\ell(w)^{2}\right), \mathcal{O}\left(\ell(w)^{3}\right)$, etc.

5.10.1 Proposition. There exists an algorithm that, given a root $\alpha$, decides whether it is periodic, critical, odd, even. Moreover, for odd roots it decides whether it is outward or inward, and for even roots it decides whether it is supporting or not. If $\operatorname{dp}(\alpha)=\mathcal{O}(\ell(w))$ then the algorithm is quadratic.

Proof. It is easy to decide whether $\alpha$ is periodic. Using condition 5.7.2(1), it is easy to decide whether $\alpha$ is critical. Both are quadratic in time by 5.9.1 and 5.9.2. Let us suppose $\alpha$ to be non-periodic. We will describe the algorithm deciding whether $\alpha$ is odd or even. First find $n>0$ such that $\left|\left(\alpha, w^{n} \alpha\right)\right| \geq 1$ by simply trying $n=1$, $2, \ldots$ By 5.3.2, such an $n$ exists and is bounded. Moreover, by 5.3.2 again, $\alpha$ is odd if $\left(\alpha, w^{n} \alpha\right) \geq 1$ and $\alpha$ is even if $\left(\alpha, w^{n} \alpha\right) \leq-1$. This enables us to distinguish between odd and even. Now suppose $\alpha$ to be odd. By 1.4.7, we have $\alpha<w^{n} \alpha$ or $w^{n} \alpha<\alpha$. By 1.4.7 (d), for all different roots $\beta$, $\gamma$, we have $\beta<\gamma$ if and only if 
$\left\langle U^{0}, \gamma-\beta\right\rangle \subset \mathbb{R}_{>0}$. Hence, choosing any point $u \in U^{0}$ (for example, $u \in C$ ), we have

$$
\alpha \text { is outward } \Longleftrightarrow \alpha<w^{n} \alpha \Longleftrightarrow\left\langle u, w^{n} \alpha-\alpha\right\rangle>0 .
$$

This provides a simple test whether $\alpha$ is outward or inward. A similar argument shows that an even root $\alpha$ is supporting if and only if $\left\langle u, \alpha+w^{n} \alpha\right\rangle>0$.

5.10.2 Definition. Let us call an outward half-space $A \subset W$ canonical if $1 \in A$ but $w^{n} \notin A$ for all $n=1,2, \ldots$. Similarly for roots.

Note that, for every root $\alpha$, the orbit $w^{\mathbb{Z}} \alpha$ contains a unique canonical root. Contrary to our classifications of roots so far, canonicity is not conjugacy covariant.

5.10.3 Proposition. There exists an algorithm that, given an outward root, decides whether it is canonical or not. The algorithm is quadratic, provided that the root has linear depth.

Proof. Let $\alpha$ be outward. Compute $n>0$ such that $\left(\alpha, w^{n} \alpha\right) \geq 1$. Then $A \subset w^{n} A$, where $A=A(\alpha)$. It follows that $A$ is canonical if and only if $1 \in A$ and for all $k=1,2, \ldots, n$ we have $w^{k} \notin A$. This is easily tested.

5.10.4 Proposition. The algorithm of 1.2.4 is cubic in $\mathrm{dp}(x)$. There exists an algorithm computing $d_{\Phi}(x, y)$ which is cubic in $\mathrm{dp}(x)+\mathrm{dp}(y)$.

Proof. As to the algorithm of 1.2.4, computing the next $x_{k}$ takes quadratic time in $\operatorname{dp}(x)$, and this has to be done about $d_{\Phi}(x, C)$ times. The other statement follows easily.

5.10.5 Proposition. There exists a cubic algorithm that finds all critical roots (up to $w^{\mathbb{Z}}$ ), all canonical outward roots, and $r(w)$. There exists an algorithm testing whether a given point in $U^{0}$ is in $Q(w)$. The latter algorithm is cubic if the point has linear depth.

Proof. The canonical outward roots can be found by computing the half-spaces $A$ with $1 \in A, w \notin A$, and testing them on outwardness (5.10.1) and canonicity (5.10.3). Since here a quadratic test must be applied $\ell(w)$ times, this is a cubic algorithm. Now $r(w)$ is simply equal to the number of canonical outward roots. By 5.5.7, $x \in Q(w)$ if and only if $d_{\Phi}(x, w x)=r(w)$, which can easily be tested in cubic time by 5.10.4. By 5.7.5, for every critical root $\alpha$, there exists $n \in \mathbb{Z}$ such that $w^{n} \alpha$ separates 1 from $w$. Thus, the critical roots are found (up to $w^{\mathbb{Z}}$ ) by testing all roots separating 1 from $w$ on criticality.

5.10.6 Proposition. There exists a cubic algorithm that computes a point $z \in Q(w)$ with $\mathrm{dp}(z)=\mathcal{O}(\ell(w))$. 
Proof. The algorithm is as follows. Choose any point $x \in U^{0}$. Compute the projection $y$ of $x$ on the intersection of the critical walls. (This is not necessary but may speed up the algorithm.) For $n=0,1,2, \ldots$, try whether the point $z_{n}=\left(1+w+\cdots+w^{n}\right) y$ is in $Q(w)$. By 5.6.5, some $z_{n}$ is in $Q(w)$, with bounded $n$. Hence the estimate of $\operatorname{dp}(z)$. Testing whether one $z_{n} \in Q(w)$ is cubic in time, by 5.10.5. Since $n$ is bounded, the complete algorithm is cubic.

5.10.7 Lemma. Let $x \in U^{0}$. Then $x \in Q^{*}(w)$ if and only if $x$ is on every critical wall and there is no supporting root separating $x$ from $w x$.

Proof. 'If'. Let $\alpha$ be supporting. We must show $x \in H(\alpha)$. If not, we have $w^{\mathbb{Z}} x \subset$ $K(-\alpha)$, since $w^{n} x$ and $w^{n+1} x$ are not separated by a supporting root. Let $f \in P[X]$ be such that $f(w) U^{0} \subset Q(w)$, see 5.6.5. Then $f(w) x \in K(-\alpha) \cap Q(w)=\varnothing$, a contradiction. Hence $x \in H(\alpha)$, which proves 'if'. The converse implication is trivial.

5.10.8 Proposition. There exists an algorithm that tests whether a given point is in $Q^{*}(w)$. The algorithm is cubic if the point has linear depth.

Proof. Lemma 5.10.7 suggests the following algorithm. Let $x \in U^{0}$ be given. By conjugating $w$, we may suppose that $x \in \bar{C}$ (in cubic time if $\operatorname{dp}(x)=\mathcal{O}(\ell(w))$ by 5.10.4). Compute the roots separating $x$ from $w x$. Decide whether one of them is supporting by 5.10.1, and (2) $\Longleftrightarrow(3)$ of 5.7.7. This is also cubic if $x$ has linear depth.

5.10.9 Proposition. There exists a cubic algorithm that computes $I \subset S, g \in W$ such that $\operatorname{Pc}(w)=g W_{I} g^{-1}$.

Proof. The algorithm is as follows. Compute a facet $X$ in $Q(w)$. Next we compute a general facet $Y \subset Q^{*}(w)$ (that is, the points in $Y$ are general) as follows. If $X$ is not general, then, since $Q^{*}(w)$ is open in the intersection of the critical walls, there exists a facet $Y \subset Q^{*}(w)$ such that $X \subset \bar{Y}$ and $\operatorname{dim} Y=\operatorname{dim} X+1$. Such a facet $Y$ can be found using 5.10.8. Going on this way, we end up with a general facet $Y \subset Q^{*}(w)$. By conjugating $w$, we may suppose that $Y \subset \bar{C}$. By 5.8.9, $\operatorname{Pc}(w)$ equals the standard parabolic closure of $w$, which can be computed using 1.3.6.

In the algorithm of 5.10.9, a general point in $Q^{*}(w)$ is computed. Conjecturally ??? (5.7.9), it is a general point in $Q(w)$. Another way of computing a general point in $Q(w)$ is suggested by the proof of 5.8.2. However, this algorithm is not as easy to implement as the algorithm of 5.10.9. This is why we prefer to use 5.8.9. 


\section{The conjugacy problem}

In 3.1.13 and 5.10.9, the conjugacy problem for Coxeter groups has been reduced to the case $\operatorname{Pc}(v)=\operatorname{Pc}(w)=W$. In this section we solve the conjugacy problem for this case.

We will treat affine and non-affine groups separately. The affine groups have been taken care of in 4.2.1. In Section 6.1 it is shown that for irreducible infinite non-affine groups, there exists a non-degenerate root basis. In Section 6.3 we assume in addition that $\operatorname{Pc}(w)=W$ and prove some crucial results. For example, for all outward $\alpha$, $\beta$ one has $\alpha<w^{n} \beta$ for almost all $n>0$ (6.3.9). In Section 6.4 we give our first polynomial solution to the conjugacy problem, with exponent $\# S+3$. In Section 6.5, it is shown, amongst others, that under the above conditions the maximum of the absolute values of the eigenvalues of $w$ is a simple eigenvalue (6.5.14). This opens the way to a cubic solution to the conjugacy problem (6.7.4). In Section 6.8 the results of Section 6.3 are applied to the computation of the algebraic rank of a Coxeter group.

\subsection{Dividing out the radical}

6.1.1 Lemma. Let $(W, S)$ be an irreducible Coxeter system associated to a root basis $(E,(\cdot, \cdot), \Pi)$. Suppose there exists $x=\sum_{\alpha \in \Pi} \lambda_{\alpha} \alpha \neq 0$ in the radical $E^{\perp}$ of $E$ such that $\lambda_{\alpha} \geq 0$ for all $\alpha \in \Pi$. Then $(W, S)$ is affine.

Proof. We may suppose $\Pi$ to be a basis of $E$. Namely, if $\Pi$ is not a basis of $E$, replace $E$ by an abstract vector space with basis $\Pi$ and a symmetric bilinear form $(\cdot, \cdot)^{\prime}$ such that $(\alpha, \beta)^{\prime}=(\alpha, \beta)$ for all $\alpha, \beta \in \Pi$. Let $A=\left(a_{s t}\right)$ be the Gram matrix. Let $(k, \ell, m)$ be the signature of $E$. Note that $m \geq 1$. Suppose that $\ell+m>1$. Then $A$ has an eigenvector $y$, independent of $x$, with eigenvalue $\lambda \leq 0$. Consider the non-negative irreducible matrix $B=1-A$. By 1.5.1, since $x$ is a non-negative eigenvector of $B$, the eigenvalue of $B$ at $x$ is greater than at $y$, contradicting $\lambda \leq 0$. Hence $\ell+m=1$, which implies that $(W, S)$ is affine.

Suppose that $(E,(\cdot, \cdot), \Pi)$ is a root basis. By dividing out $E^{\perp}$, we obtain a triple $\left(E / E^{\perp},(\cdot, \cdot), \Pi^{\prime}\right)$. We ask ourselves whether the new triple is still a root basis. Of the axioms for a root basis 1.2.1, parts (1) and (2) certainly hold.

6.1.2 Proposition. Let $(W, S)$ be an irreducible non-affine Coxeter system. If the triple $(E,(\cdot, \cdot), \Pi)$ is associated with $(W, S)$ and satisfies (1) and (2) of Definition 1.2.1 then it satisfies (3) as well.

Proof. Suppose that (3) is not satisfied. Then there are $\lambda_{\alpha} \geq 0(\alpha \in \Pi)$, not all zero, such that $\sum_{\alpha \in \Pi} \lambda_{\alpha} \alpha=0$. There exists a vector space $E^{\prime}$ with a basis $\Pi^{\prime}$ and a bijective map $\phi: \Pi^{\prime} \rightarrow \Pi$. Let $L: E^{\prime} \rightarrow E$ be the unique linear map such that 
$L(\alpha)=\phi(\alpha)$ for all $\alpha \in \Pi^{\prime}$. For $u, v \in E^{\prime}$ define $(u, v):=(L u, L v)$. The element $\sum_{\alpha \in \Pi} \lambda_{\alpha} \phi^{-1}(\alpha)$ is in the radical of $E^{\prime}$. This contradicts 6.1.1 and finishes the proof.

6.1.3 Proposition. Let $(E,(\cdot, \cdot), \Pi)$ be a root basis, associated to an irreducible non-affine Coxeter system $(W, S)$. Then by dividing out the radical $E^{\perp}$, one obtains again a root basis associated to $(W, S)$.

Proof. Immediate from 6.1.2.

Proposition 6.1.3 is implicit in the work of Vinberg, [47], Proposition 13, p. 1100.

6.1.4 Example. By 6.1.3, every irreducible non-affine Coxeter group is associated to a non-degenerate root basis $(E,(\cdot, \cdot), \Pi)$. We cannot suppose $\Pi$ to be independent, as is shown by the following example. Let $S=\mathbb{Z} / n, m_{s t}=2$ if $s-t \neq \pm 1, m_{s t}=4$ if $s-t= \pm 1$. Then $\sum_{s \in S} a_{s} e_{s} \in E^{\perp}$ if and only if $a_{s}-\left(a_{s-1}+a_{s+1}\right) / \sqrt{2}=0$ for all $s \in S$, or, equivalently,

$$
\left(\begin{array}{c}
a_{s} \\
a_{s+1}
\end{array}\right)=\left(\begin{array}{cc}
0 & 1 \\
-1 & \sqrt{2}
\end{array}\right)\left(\begin{array}{c}
a_{s-1} \\
a_{s}
\end{array}\right)
$$

Since the above $2 \times 2$ matrix has order 8 , the classical root basis is degenerate if $n$ is an 8 -tuple. Suppose that $n$ is an 8-tuple. Then the radical of the classical root basis has dimension 2. Since all $m_{s t}$ are finite, every root basis (spanned by $\Pi$ ) is a quotient of the classical root basis. Hence in every non-degenerate root basis $\Pi$ is dependent.

\subsection{Ordered triples of roots}

6.2.1 Lemma. A root basis $(E,(\cdot, \cdot), \Pi)$ with $E=\operatorname{Span}(\Pi)$ cannot have signature $(1,2)$ or $(1,1,1)$.

Proof. Suppose that $\operatorname{dim} E=3$. Let $\{\alpha, \beta, \gamma\} \subset \Pi$ be a basis of $E$. Let $A$ be the Gram matrix with respect to this basis. Thus,

$$
A=\left(\begin{array}{rrr}
1 & -a & -c \\
-a & 1 & -b \\
-c & -b & 1
\end{array}\right), \quad a, b, c \geq 0 .
$$

If one of $a, b, c$ is smaller than 1 , say $a<1$, then there is a subspace $\operatorname{Span}\{\alpha, \beta\}$ of signature $(2,0)$, which makes signatures $(1,2)$ and $(1,1,1)$ impossible. Otherwise, since $a, b, c \geq 1$, we have

$$
\operatorname{det}(A)=1-2 a b c-\left(a^{2}+b^{2}+c^{2}\right) \leq 1-2-3<0,
$$

which again makes signatures $(1,2)$ and $(1,1,1)$ impossible. 
6.2.2 Proposition. Let $\alpha \leq \beta \leq \gamma$ be roots. Let us write $2(\alpha, \beta)=p+p^{-1}$, $2(\beta, \gamma)=q+q^{-1}, 2(\alpha, \gamma)=r+r^{-1}, p, q, r \geq 1$. Then $r \geq p q$.

Proof. By 1.4.7 (e), we may suppose $\Phi$ to be generated by $\{\alpha, \beta, \gamma\}$. Assume also that $E=\operatorname{Span} \Phi$. Then $\operatorname{dim} E \leq 3$. If $E$ is positive definite or semi-definite, then for all $x, y \in E$ with $(x, x)=(y, y)=1$ we have $|(x, y)| \leq 1$. Hence $p=q=r=1$, and the result follows. Thus, suppose that $E$ is not positive definite nor semi-definite. By 6.2.1 (and noting that $E$ contains a positive definite subspace $\mathbb{R} \alpha$ ), $E$ has signature $(2,1)$ or $(1,1)$.

Since $E$ is non-degenerate, it may be identified to its dual. Let $H=\{x \in E$ $(x, x)<0\}$. Let $H^{+}, H^{-}$be the two connected components of $H$. By 4.6.1, $U^{0}$ contains either $H^{+}$or $H^{-}$.

There are two ways of finishing the proof. The first way uses the geometry of the hyperbolic plane, as follows. Suppose $E$ to have signature $(2,1)$, and suppose that $p, q, r>1$. Since $U^{0}$ contains $H^{-}$or $H^{-}, \alpha, \beta$ and $\gamma$ define three parallel geodesics in $\mathbb{H}^{2}$, ordered in this order. Let us denote these geodesics by $\alpha^{\perp}, \beta^{\perp}, \gamma^{\perp}$. It is known [5], Corollary A.5.8, that the hyperbolic distance between $\alpha^{\perp}$ and $\beta^{\perp}$ is $\log (p)$, and similarly for the other pairs of geodesics. The (unique) shortest path from $\alpha^{\perp}$ to $\gamma^{\perp}$ intersects $\beta^{\perp}$. This shows that $\log (r) \geq \log (p)+\log (q)$, whence $r \geq p q$. The cases where $E$ has signature $(1,1)$, or one of $p, q, r$ equals 1 , are left to the reader in our first end of the proof.

The second way of finishing the proof will not depend on any knowledge of the hyperbolic plane.

Write $a=(\alpha, \beta), b=(\beta, \gamma), c=(\alpha, \gamma)$. Let $A$ be the Gram matrix with respect to $\alpha, \beta, \gamma$ :

$$
A=\left(\begin{array}{lll}
1 & a & c \\
a & 1 & b \\
c & b & 1
\end{array}\right), \quad a, b, c \geq 0 .
$$

We have

$$
0 \leq-\operatorname{det}(A)=c^{2}-2 a b c+\left(a^{2}+b^{2}-1\right),
$$

whence

$$
c \leq a b-\sqrt{\left(a^{2}-1\right)\left(b^{2}-1\right)} \quad \text { or } \quad c \geq a b+\sqrt{\left(a^{2}-1\right)\left(b^{2}-1\right)} .
$$

Note that we may assume $a>1$ or $b>1$, for otherwise we have $p=q=1$ and certainly $r \geq p q$. By symmetry, we may assume $a>1$.

We saw that $U^{0}$ contains either $H^{+}$or $H^{-}$. Combining with 1.4.7(d), it follows that the sequence $(x, \alpha),(x, \beta),(x, \gamma)$ is monotonic for all $x \in H$. We apply this to $x=\alpha-\beta$. We have $(x, x)=2-2 a<0$, whence indeed $x \in H$. By the above, the sequence $((x, \alpha),(x, \beta),(x, \gamma))=(1-a, a-1, c-b)$ is monotonic. Since it was assumed that $a>1$, we find it to be increasing, and hence $a-1 \leq c-b$, or

$$
c \geq a+b-1 \text {. }
$$


If $b=1$, then from (10), we find $c \geq a$, which gives the desired result. Therefore, let us assume that $a, b>1$. We will show that (10) contradicts the first possibility of (9). These two inequalities would imply successively

$$
\begin{aligned}
a+b-1 & \leq c \leq a b-\sqrt{\left(a^{2}-1\right)\left(b^{2}-1\right)}, \\
\sqrt{\left(a^{2}-1\right)\left(b^{2}-1\right)} & \leq a b-(a+b-1), \\
\left(a^{2}-1\right)\left(b^{2}-1\right) & \leq(a-1)^{2}(b-1)^{2},
\end{aligned}
$$

and as $a, b>1$,

$$
(a+1)(b+1) \leq(a-1)(b-1),
$$

a contradiction indeed. Hence the second possibility of (9) holds. In terms of $p, q, r$ one finds

$$
\begin{aligned}
c & \geq \frac{\left(p+p^{-1}\right)\left(q+q^{-1}\right)+\sqrt{\left(\left(p+p^{-1}\right)^{2}-4\right)\left(\left(q+q^{-1}\right)^{2}-4\right)}}{4} \\
& =\frac{\left(p+p^{-1}\right)\left(q+q^{-1}\right)+\left(p-p^{-1}\right)\left(q-q^{-1}\right)}{4}=\frac{p q+(p q)^{-1}}{2} .
\end{aligned}
$$

Hence $r \geq p q$.

6.2.3 Corollary. Let $\alpha \leq \beta \leq \gamma$ be roots. Then

$$
(\alpha, \beta) \leq(\alpha, \gamma)
$$

and

$$
(\beta, \gamma) \leq(\alpha, \gamma)
$$

Proof. This follows from 6.2.2 and the fact the map $x \mapsto x+x^{-1}$ is increasing for $x \geq 1$.

6.3. Outward roots and their ordering. In this section we assume $(W, S)$ to be irreducible, infinite and non-affine, unless stated otherwise. Furthermore, an element $w \in W$ with $\operatorname{Pc}(w)=W$ is fixed. By 5.8.7, we have $\operatorname{Pc}^{\infty}(w)=W$, and $\operatorname{Span}(\Pi)$ is spanned by the outward roots.

By 6.1.3 there exists a non-degenerate root basis $(E,(\cdot, \cdot), \Pi)$ for $W$. Also we may assume that $E$ is spanned by $\Pi$ and hence by the odd roots. Such $E$ is fixed from now on.

One of the main results of this section is Corollary 6.3.8 stating that for all outward $\alpha, \beta$ one has $\left(\alpha, w^{n} \beta\right) \rightarrow \infty$ as $n \rightarrow \infty$ or $n \rightarrow-\infty$. A result of independent interest is Corollary 6.3.10 stating that $w^{\mathbb{Z}}$ has finite index in the centralizer of $w$.

6.3.1 Lemma. Let $\alpha, \beta$ be $w$-outward. Then the following holds. 
(a) $(\alpha, \beta)>-1$.

(b) For almost all $n \in \mathbb{N},\left(\alpha, w^{n} \beta\right) \geq 1$ implies that $\alpha<w^{n} \beta$.

Proof. Part (a) is left to the reader (use 1.4.7 (b) and the definition of outwardness). In order to prove (b), let $x \in U^{0}$ be any point. By definition of outwardness, $\left\langle x, w^{n} \beta\right\rangle>$ 0 for almost all $n \in \mathbb{N}$. By 5.7.1, $\lim _{n \rightarrow \infty}\left\langle x, w^{n} \beta\right\rangle=\infty$. Hence for almost all $n \in \mathbb{N},\left\langle x, w^{n} \beta-\alpha\right\rangle>0$. For these $n$, the additional condition $\left(\alpha, w^{n} \beta\right) \geq 1$ implies, by 1.4.7(b), that $\alpha<w^{n} \beta$.

6.3.2 Lemma. Let $\alpha, \beta$ be outward roots such that $\left(\beta, w^{n} \alpha\right)$ is unbounded $(n \in \mathbb{N})$. Then there exists $k \in \mathbb{N}$ such that $w^{-k} \alpha<\beta$.

Proof. Recall that $(\Phi, \Phi) \cap(-1,1)$ is finite by 3.2.1(a). For infinitely many $k \in \mathbb{N}$ we have $\left|\left(w^{-k} \alpha, \beta\right)\right| \geq 1$ because otherwise the sequence $\left(w^{n} \alpha, \beta\right)$ would be periodic, contradicting its unboundedness. By 6.3.1(a) there is an infinite set $L \subset \mathbb{N}$ such that $\left(w^{-k} \alpha, \beta\right) \geq 1$ for all $k \in L$. By 6.3.1(b) almost all $k \in L$ satisfy $\alpha<w^{k} \beta$.

6.3.3 Lemma. Let $\alpha$ be odd. Then $\left(\alpha, w^{n} \alpha\right)$ is unbounded $(n \in \mathbb{N})$.

Proof. Since $W$ is irreducible and non-affine, we may assume that $E$ is non-degenerate by 6.1.3. Also, we may assume that $E$ is spanned by $\Pi$ and hence by the odd roots.

The set $w^{\mathbb{N}} \alpha$ is a discrete subset of $E$ by 1.2 .5 and it is infinite since $\alpha$ is odd. Hence it is unbounded. Since the odd roots span $E$, and $E$ is non-degenerate, there exists an odd root $\beta$ such that $\left(\beta, w^{n} \alpha\right)$ is unbounded $(n \in \mathbb{N})$. Suppose, as we may, that $\alpha$ and $\beta$ are outward. By 6.3.2, we have $w^{-k} \alpha<\beta$ for some $k \in \mathbb{N}$. By 6.3.1, there exists $M>0$ such that for all $n$ with $n>M$ and $\left(\beta, w^{n} \alpha\right) \geq 1$, we have $\beta<w^{n} \alpha$. In order to show that $\left(\alpha, w^{n} \alpha\right)$ is unbounded, let $N \geq 1$. Let $n>M$ be such that $\left(\beta, w^{n} \alpha\right) \geq N-$ see 6.3.1 (a). Thus, $w^{-k} \alpha<\beta<w^{n} \alpha$. By 6.2.3, we have $\left(\alpha, w^{k+n} \alpha\right)=\left(w^{-\bar{k}} \alpha, w^{n} \alpha\right) \geq\left(\beta, w^{n} \alpha\right) \geq N$.

The preceding lemma is a crucial step to our results. Notice the similarity to 5.3.1. The result of 6.3.3 however does not hold for affine groups. If the root basis is positive semi-definite, then for all roots $\alpha, \beta$, we have $|(\alpha, \beta)| \leq 1$.

6.3.4 Definition. Let us call two odd roots $\alpha, \beta$ equivalent, notation $\alpha \sim \beta$, if $\left(\alpha, w^{n} \beta\right)$ is unbounded, $n \in \mathbb{N}$.

6.3.5 Lemma. Equivalence of odd roots is an equivalence relation.

Proof. Reflexivity. This is the content of 6.3.3.

Symmetry. Let $\alpha \sim \beta$. We may suppose $\alpha$ and $\beta$ to be outward. Let $N>1$. By 6.3.2, applied to $\alpha, \beta$ interchanged, there exists $k \in \mathbb{N}$ such that $\beta<w^{k} \alpha$. By 
6.3.3 and 6.3.1 (a), there exists $n>0$ such that $\left(\beta, w^{n} \beta\right) \geq N$. Note that $\beta<w^{n} \beta$. Applying 6.2.3 to the sequence $\beta<w^{n} \beta<w^{n+k} \alpha$ we obtain $\left(\beta, w^{n+k} \alpha\right) \geq$ $\left(\beta, w^{n} \beta\right) \geq N$. Hence $\beta \sim \alpha$.

Transitivity. Let $\alpha \sim \beta$ and $\beta \sim \gamma$. We may suppose $\alpha, \beta$ and $\gamma$ to be outward. Let $N \geq 1$. By 6.3.1 (b), there exist $m>n>0$ such that $\alpha<w^{n} \beta<w^{m} \gamma$, $\left(\alpha, w^{n} \beta\right) \geq N$. By 6.2.3, we have $\left(\alpha, w^{m} \gamma\right) \geq\left(\alpha, w^{n} \beta\right) \geq N$ and so $\alpha \sim \gamma$.

The proof of the following theorem uses symmetry as well as transitivity of $\sim$ (6.3.5).

6.3.6 Theorem. Let $\alpha, \beta$ be odd. Then $\alpha \sim \beta$ if and only if $w^{\mathbb{Z}} \alpha \not \perp \beta$.

Proof. 'Only if' is trivial. In order to prove 'if', one may assume $(\alpha, \beta) \neq 0$, for otherwise, replace $\beta$ by some $w^{n} \beta$. Suppose that $\alpha \nsim \beta$. By symmetry of the equivalence relation $\sim(6.3 .5)$, the sequence $\left(\alpha, w^{n} \beta\right)$ is bounded $(n \in \mathbb{Z})$. Consider the root

$$
\gamma=r_{\beta} \alpha=\alpha-2(\alpha, \beta) \beta=: \alpha+\lambda \beta .
$$

We will show that $\gamma$ is odd. We have

$$
\left(\gamma, w^{n} \gamma\right)=\left(\alpha+\lambda \beta, w^{n}(\alpha+\lambda \beta)\right)=\left(\alpha, w^{n} \alpha\right)+\lambda^{2}\left(\beta, w^{n} \beta\right)+\text { bounded. }
$$

We have $\left(\alpha, w^{n} \alpha\right),\left(\beta, w^{n} \beta\right)>-1$ for all $n$ by 6.3.1 (a). Moreover, by 6.3.3, we have $\lim \sup _{n \rightarrow \infty}\left(\alpha, w^{n} \alpha\right)=\infty$. It follows that $\lim \sup _{n \rightarrow \infty}\left(\gamma, w^{n} \gamma\right)=\infty$, so $\gamma$ is odd by 5.3.2. Now $\gamma$ is equivalent to $\alpha$ as well as $\beta$, because

$$
\begin{aligned}
& \left(\alpha, w^{n} \gamma\right)=\left(\alpha, w^{n} \alpha\right)+\text { bounded, } \\
& \left(\beta, w^{n} \gamma\right)=\lambda\left(\beta, w^{n} \beta\right)+\text { bounded }
\end{aligned}
$$

and $\lambda \neq 0$. Hence $\alpha$ and $\beta$ are equivalent, a contradiction. This finishes the proof.

\subsubsection{Corollary. Two odd roots are always equivalent.}

Proof. Suppose that the set of odd roots can be written $X \sqcup Y, X, Y \neq \varnothing$ and $X \perp Y$. Since $\Phi$ is generated by the odd roots, it would follow that $\Phi$ is reducible, a contradiction. Hence a partition as above is impossible. By 6.3.6, two odd roots are always equivalent.

6.3.8 Corollary. Let $\alpha, \beta$ be outward. Then $\left(\alpha, w^{n} \beta\right) \rightarrow \infty$ as $n \rightarrow \infty$ or $n \rightarrow-\infty$.

Proof. By 6.3.3, there exists $m>0$ such that $\left(\alpha, w^{m} \alpha\right)>1$. So $\alpha<w^{m} \alpha$. By applying 6.2.2 inductively to the sequences $\alpha<w^{n m} \alpha<w^{(n+1) m} \alpha$, we find that $\left(\alpha, w^{n m} \alpha\right) \rightarrow \infty$ as $n \rightarrow \infty$. It is enough to show that for all $r \in \mathbb{N}$ we have 
$\left(\alpha, w^{n m+r} \beta\right) \rightarrow \infty$ as $n \rightarrow \infty$; the case $n \rightarrow \infty$ follows by interchanging $\alpha$ and $\beta$. Fix such an $r$. Note that $\operatorname{Pc}\left(w^{m}\right)=W$ since $\operatorname{Pc}\left(w^{m}\right) \supset \operatorname{Pc}^{\infty}\left(w^{m}\right)=\operatorname{Pc}^{\infty}(w)=W$. By applying 6.3.7 and 6.3.1 to $w^{m}$ instead of $w$ and the $w^{m}$-outward roots $\alpha$ and $w^{r} \beta$, we find that $\alpha<w^{k m+r} \beta$ for some $k \in \mathbb{Z}$. By 6.2.3 applied to the sequence $\alpha<w^{n m} \alpha<w^{(n+k) m+r} \beta$, we find $\left(\alpha, w^{(n+k) m+r} \beta\right) \geq\left(\alpha, w^{n m} \alpha\right) \rightarrow \infty$ as $n \rightarrow \infty$.

6.3.9 Corollary. Let $\alpha, \beta$ be outward. Then $w^{-n} \alpha<\beta<w^{n} \alpha$ for almost all $n \in \mathbb{N}$.

Proof. This follows from 6.3.8 and 6.3.1.

6.3.10 Corollary. We have $\left[Z(w): w^{\mathbb{Z}}\right]<\infty$.

Proof. Recall the set $\operatorname{Out}(w)$ of outward roots. The group $Z(w)$ permutes the finite set $w^{\mathbb{Z}} \backslash \operatorname{Out}(w)$. Let $G$ be the kernel of this action. Thus, $[Z(w): G]<\infty$. We will prove that $G=w^{\mathbb{Z}}$. Let $g \in G$. Choose any outward root $\alpha$. By multiplying $g$ by a power of $w$, we may suppose that $g \alpha=\alpha$. Let $\beta$ be an outward root and write $g \beta=w^{k} \beta$. We have for all $n \in \mathbb{Z}$ :

$$
\left(\alpha, w^{n} \beta\right)=\left(g \alpha, g w^{n} \beta\right)=\left(\alpha, w^{n} g \beta\right)=\left(\alpha, w^{n+k} \beta\right) .
$$

By 6.3.8 however, $\left(\alpha, w^{n} \beta\right)$ is unbounded. Thus $k=0$, whence $g \beta=\beta$. Since the outward roots span $E$, it follows that $g=1$.

The above proof in fact shows that the $Z(w) / w^{\mathbb{Z}}$-action on $w^{\mathbb{Z}} \backslash$ Out $(w)$ is faithful. Corollary 6.3.10 was subsequently used in [13], [18], [15].

In the proof of the following theorem, the root basis $E / E^{\perp}$ is used once again.

6.3.11 Theorem. Let $F$ denote the set of w-periodic roots. Then $F$ is a finite parabolic root subsystem of $\Phi$.

Proof. As in the proof of 6.3.3, we may assume $E$ to be non-degenerate and spanned by the odd roots. We will prove that for every periodic root $\alpha$ and odd root $\beta$, we have $|(\alpha, \beta)|<1$. Suppose the contrary. We may assume $\beta$ to be outward and $\alpha<\beta$; see 1.4.7(b). Choose any point $x \in A(\alpha)$. Let $k>0$ be such that $w^{k} \alpha=\alpha$. Let $n>0$ be such that $w^{k n} x \in A(-\beta)$. Then $w^{k n} x \in A(-\alpha)$. Hence $x \in A\left(-w^{-k n} \alpha\right)=A(-\alpha)$, a contradiction. We conclude $|(\alpha, \beta)|<1$. Since $E$ is non-degenerate and the odd roots span $E$, the set $F$ is bounded. Since $F$ is discrete by 5.7.1, it is finite.

It is easy to see that $F$ is a root subsystem and that $F=\operatorname{Span}(F) \cap \Phi$. By 3.2.3, $F$ is a parabolic root subsystem of $\Phi$. 
6.3.12 Corollary. Let $W$ be a Coxeter group. Then there exists a constant $N=N(W)$ such that for all $w \in W$ and every $w$-periodic root $\alpha$, we have $w^{N} \alpha=\alpha$. The constant $N$ may be taken to be the least common multiple of the orders of elements of

$$
N_{\mathrm{O}\left(E_{I}\right)}\left(W_{I}\right)
$$

for all spherical I $\subset S$.

Proof. Let $\alpha$ be $w$-periodical. By 5.8.5, we have either $\alpha \perp \Phi_{\text {odd }}$ or $\alpha \in \Phi_{\text {odd }}$. First, suppose that $\alpha \perp \Phi_{\text {odd }}$. Since $\operatorname{Pc}(w)=\operatorname{Pc}^{\infty}(w) \times \operatorname{Pc}^{0}(w)(5.8 .7)$ and $\operatorname{Pc}^{0}(w)$ is a finite parabolic subgroup (5.7.6), we have $w^{N} \in \operatorname{Pc}^{\infty}(w)$. Hence $w^{N} \alpha=\alpha$.

Next suppose that $\alpha \in \Phi_{\text {odd }}$. Let $P$ be the component of $\mathrm{Pc}^{\infty}(w)$ containing $r_{\alpha}$. We may suppose $P$ to be standard parabolic, say $P=W_{J}$. Let $\bar{w}$ denote the projection of $w$ on $W_{J}$. Note that $W_{J}$ is infinite, by 5.8.7. We consider separately the cases of $W_{J}$ affine or non-affine.

If $W_{J}$ is affine, let $T \subset W_{J}$ be the translation subgroup. Then $W_{J} / T \cong W_{I}$ for some $I \subset J$. Let $\pi: W_{J} \rightarrow W_{I}$ be the projection. Then $(\pi \bar{w})^{N}=1$ because

$$
\pi \bar{w} \in W_{I} \subset N_{\mathrm{O}\left(E_{I}\right)}\left(W_{I}\right) .
$$

Hence $\bar{w}^{N}$ is a translation. Since $\alpha$ is $\bar{w}$-periodic, we must have $\bar{w}^{N} \alpha=\alpha$, whence $w^{N} \alpha=\alpha$.

Now suppose $W_{J}$ to be non-affine. Note that $W_{J}=\operatorname{Pc}(\bar{w})$. Hence, by 6.3.11, the set of $\bar{w}$-periodic roots in $\Phi_{J}$ is a finite parabolic root subsystem of $\Phi_{J}$, say $\Phi_{I}$, $I \subset J$. Since

$$
\left.w\right|_{E_{I}} \in N_{\mathrm{O}\left(E_{I}\right)}\left(W_{I}\right)
$$

we find that $w^{N} \alpha=\alpha$.

6.4. First solution to the conjugacy problem. In the following results $6.4 .1,6.4 .2$ and 6.4.3, we suppose $W$ to be irreducible, infinite, non-affine.

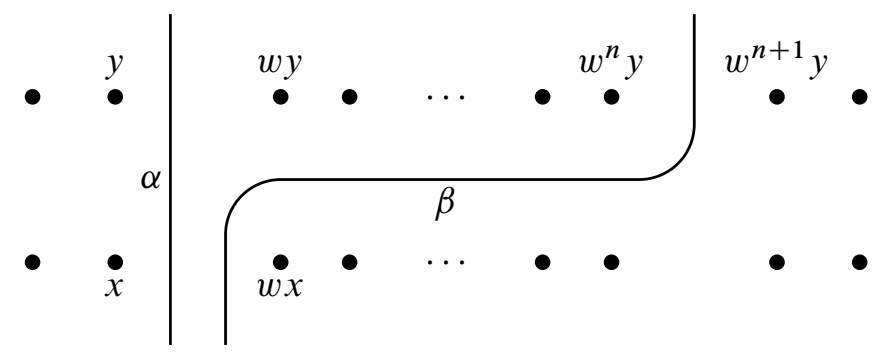

Figure 9. Illustration for 6.4.1.

The following lemma says that two odd walls cannot be too far from being parallel in some sense. Recall $c:=\max (2, \#\{(\alpha, \beta) \mid \alpha, \beta \in \Phi\} \cap(-1,1))$. 
6.4.1 Lemma. There exists a constant $N=N(W)$ such that the following holds. Let $w \in W$ with $\operatorname{Pc}(w)=W$. Let $\alpha, \beta$ be two $w$-outward roots, and let $x, y \in Q(w)$, $n \in \mathbb{Z}$ be such that $\alpha$ and $\beta$ separate $x$ from $w x, \alpha$ separates $y$ from $w y$ and $\beta$ separates $w^{n} y$ from $w^{n+1} y$, as is shown in Figure 9. (Separation need not be strict.) Then $|n| \leq N$. More precisely, $N$ may be taken to be $(c+2)^{r}+r$.

Proof. We may assume that $n \geq 0$. We have $x \in K(\alpha) \cap K\left(-w^{-k} \beta\right)$, wy $\in$ $K(-\alpha) \cap K\left(w^{-k} \beta\right)$ for every $k$ with $1 \leq k \leq n-1$. Since $\alpha$ and $\beta$ are outward, $K(\alpha) \cap K\left(w^{-k} \beta\right)$ and $K(-\alpha) \cap K\left(-w^{-k} \beta\right)$ are non-empty as well. It follows that either $\left|\left(\alpha, w^{-k} \beta\right)\right|<1$ or $\alpha= \pm w^{-k} \beta$ by 1.4.7 (b). Thus, $\left|\left(\alpha, w^{-k} \beta\right)\right| \leq 1$. Write $\left(\alpha, w^{-k} \beta\right)=a_{k}$. Consider the $r$-tuples $\left(a_{m+1}, \ldots, a_{m+r}\right)$ where $1 \leq m+1$, $m+r \leq n-1$. These $r$-tuples are different because $\left(a_{k}\right)$ is not periodic by 6.3.8. Thus, the number of $r$-tuples equals $n-r \leq(c+2)^{r}$, whence $n \leq(c+2)^{r}+r$.

The following result roughly says that 'slices' of $Q(w)$ have diameter $\mathcal{O}(\ell(w))$ provided that $\operatorname{Pc}(w)=W$.

6.4.2 Theorem. There exists a constant $N=N(W)$ such that the following holds. Let $w \in W$ be such that $\operatorname{Pc}(w)=W$. Let $x, y \in Q(w)$ and suppose that there exists an odd root separating $x$ from $w x$ and $y$ from $w y$. Then $d_{\Phi}(x, y) \leq N \ell(w)$.

Proof. Let $\alpha$ be an odd root separating $x$ from $w x$ and $y$ from $w y$. We start by showing that $d_{\Phi}(x, y)$ equals the number of outward roots strictly separating $x$ from $y$ plus a bounded number. By 6.3.11, the number of periodic roots is bounded by $\max \left\{\# \Phi_{I} \mid I \subset S\right.$ spherical $\}$. There are no even roots separating $x$ from $y$ because if $\beta$ is even then either $H(\beta)$ or $H(-\beta)$ contains $Q(w)$ and hence $x$ and $y$. Finally, there are only boundedly many roots non-strictly separating $x$ from $y$. Thus, one needs only bound the number of outward roots strictly separating $x$ from $y$.

Let $\beta$ be such a root. Let $k, \ell$ be integers such that $\beta$ separates $w^{k} x$ from $w^{k+1} x$ and $w^{\ell} y$ from $w^{\ell+1} y$. Here separation need not be strict. Applying 6.4.1 to $\alpha$ and $w^{-k} \beta$ shows

$$
|k-\ell| \leq N_{0}:=(c+2)^{r}+r .
$$

We will now show that

$$
\left(k+\frac{1}{2}\right)\left(\ell+\frac{1}{2}\right)<0 .
$$

First suppose that $k, \ell \geq 0$. Then $x, y \in K(\beta)$, contradicting the assumption that $\beta$ strictly separates $x$ from $y$. Similarly, it is disproved that $k, \ell<0$, which proves (12). From (11) and (12), it follows that $k$ and $\ell$ are bounded, and more precisely $-N_{0} \leq k \leq N_{0}-1$. Thus, $\beta$ separates $w^{-N_{0}} x$ from $w^{N_{0}} x$. We conclude that

$$
d_{\Phi}(x, y) \leq 2 N_{0} r(w)+\text { a bounded number }=\mathcal{O}(\ell(w)) .
$$


6.4.3 Lemma. There exists an algorithm that, given $w \in W$ with $\operatorname{Pc}(w)=W$ and $w$-outward roots $\alpha, \beta$ separating 1 from $w$, determines the smallest integer $n$ such that $\alpha \leq w^{n} \beta$. If $\alpha$ and $\beta$ have linear depth, then the complexity is quadratic.

Proof. Note that $n \geq 0$. Thus, one algorithm runs by simply trying $n=0,1, \ldots$, using 1.4.7 (d) to decide whether $\alpha \leq w^{n} \beta$. The smallest $n$ satisfying this is what we look for. By an argument similar to 5.3.2, $n$ is bounded. For each $n$, quadratic time is needed, whence the complexity is quadratic.

6.4.4 Theorem. Coxeter groups have polynomial time conjugacy problem, with exponent $r+3=\# S+3$.

Proof. The algorithm is as follows. Let $v, w \in W$. By 5.10.9, compute the parabolic closures of $v$ and $w$. By 3.1.13, it is enough to solve the conjugacy problem for the case $\operatorname{Pc}(v)=\operatorname{Pc}(w)=W$. We may suppose $W$ to be irreducible and infinite. By 4.2.1, we may suppose $W$ to be non-affine. Choose a non-degenerate root basis for $W$ - see 6.1.3. By 5.10.6, compute points $x \in Q(w), z \in Q(v)$ of linear depth. By conjugating $v$ and $w$, we may suppose that $x, z \in \bar{C}$. Compute the canonical $v$-odd and $w$-odd roots by 5.10.5. Let $\alpha_{1}, \ldots, \alpha_{k}(k \leq r)$ be a basis of $E$ consisting of $v$-outward roots, and suppose that $\alpha_{1}$ separates $z$ from $v z$. Let $\beta_{1}, \ldots, \beta_{n}(n=r(w))$ denote the $w$-outward roots separating $x$ from $w x$ and with $x \in H\left(\beta_{i}\right)$. The algorithm so far takes cubic time. If some $\beta_{i}$ separates $w^{m}$ from $w^{m+1}$ for some $m$, then $m$ is bounded. The same may be supposed to hold for the $\alpha_{i}$. Hence, in $\mathcal{O}\left(\ell(w)^{2} n^{2}\right)=\mathcal{O}\left(\ell(w)^{4}\right)$ time, we can compute the smallest integers $p(i, j), q(i, j)$ such that $\alpha_{i} \leq v^{p(i, j)} \alpha_{j}, \beta_{i} \leq w^{q(i, j)} \beta_{j}$ by 6.4.3.

We postpone the continuation of the algorithm for a theoretical observation. If $v$ and $w$ are conjugate, then there exists $g$ with $g v g^{-1}=w$ and $g \alpha_{1}=\beta_{i}$ for some $i$, after multiplying $g$ at the left by a suitable power of $w$. Let $X$ denote the set of injective maps $\phi:\left\{v^{\mathbb{Z}} \alpha_{i} \mid i=1, \ldots, k\right\} \rightarrow w^{\mathbb{Z}} \backslash \operatorname{Out}(w)$. Each $g \in W$ with $g v g^{-1}=w$ induces an element of $X$. We will show that $g$ is determined by its image in $X$ and the condition that there exists $i$ with $g \alpha_{1}=\beta_{i}$. Let $m, j$ be such that $m>1$ and $g v^{\mathbb{Z}} \alpha_{m}=w^{\mathbb{Z}} \beta_{j}$. Since $p(1, m)$ is the smallest integer with $\alpha_{1} \leq v^{p(1, m)} \alpha_{m}$ and $q(i, j)$ is the smallest integer with $g \alpha_{1}=\beta_{i} \leq w^{q(i, j)} \beta_{j}$, we must have $g \alpha_{m}=w^{q(i, j)-p(1, m)} \beta_{j}$. This determines $g$, because $E$ is spanned by the $\alpha_{m}$.

Thus, the algorithm may be continued as follows. For each $\phi \in X$, do the following. Compute $A \in \operatorname{End}(E)$ such that

(1) $A \alpha_{1}=\beta_{i}$ where $\phi\left(v^{\mathbb{Z}} \alpha_{1}\right)=w^{\mathbb{Z}} \beta_{i}$;

(2) if $\phi\left(v^{\mathbb{Z}} \alpha_{m}\right)=w^{\mathbb{Z}} \beta_{j}(m>1)$ then $A \alpha_{m}=w^{q(i, j)-p(1, m)} \beta_{j}$.

Next check whether $A v=w A$. It may also be checked whether $A \in \mathrm{O}(E,(\cdot, \cdot))$. This is not necessary but may speed up the algorithm. If one of these conditions 
is not fulfilled, $A$ is left from consideration. Now, one must decide whether or not $A \in W$, where $W$ is viewed as a subgroup of GL(E). Suppose that $A \in W$. Write $y=A z \in A Q(v)=Q(w)$. Note that $A \alpha_{1}$ separates $x$ from $w x$ and $y$ from $w y$. By 6.4.2, $\ell(A) \leq M:=N \ell(w)$ for some (known) constant $N$. This shows how to decide whether $A \in W$. Namely, compute $A=A_{0}, A_{1}, \ldots$ which are defined by $A_{i+1}=s A_{i}$, where $s \in S$ such that $\left\langle A_{i} C, e_{s}\right\rangle \subset \mathbb{R}_{<0}$. Then $A \in W$ if and only if we have $A_{i}=I$ for some $i \leq M$.

Now $v$ and $w$ are conjugate if and only if for at least one $\phi \in X$, we have $A v A^{-1}=w$ and $A \in W$. Note that $\# X=n(n-1)(n-2) \ldots(n-k+1) \leq$ $n^{k}=r(w)^{k} \leq \ell(w)^{r}$. Since checking one $A$ takes cubic time (compare 5.10.4), the algorithm has polynomial complexity of degree $\max (4, r+3)$ and hence $r+3$ (if $4>r+3$, then the theorem is trivial).

6.4.5 Remark. The complexity of the algorithm of 6.4.4 depends (not only theoretically but also in practice) on the constant of 6.4.2, namely, in the process of deciding whether $A \in W$. This constant is very large. This is an important practical disadvantage of 6.4.4.

6.4.6 Lemma. Let $G=\mathbb{Z}^{n} \rtimes F$ be the semi-direct product of $\mathbb{Z}^{n}$ and a finite group $F$ acting on $\mathbb{Z}^{n}$. Let $\ell$ be the length function with respect to some finite generating subset of $G$. Then there exists a constant $N$ such that if $v, w \in W$ are conjugate, then there exists $g \in W$ with $g v g^{-1}=w$ and $\ell(g) \leq N(\ell(v)+\ell(w))$.

Proof. The proof goes along the same lines as 4.2.1 and is left to the reader.

The following corollary obviously suggests an algorithm solving the conjugacy problem. However, this solution is exponential in general. We include the corollary because of interest in its own. The proof is similar to that of 6.4.4.

6.4.7 Corollary. Let $W$ be a ( finite rank) Coxeter group. Then there exists a constant $N$ such that if $v, w \in W$ are conjugate, then there exists $g \in W$ with $g v g^{-1}=w$ and $\ell(g) \leq N(\ell(v)+\ell(w))$.

Proof. Let $v, w \in W$ be conjugate. Since $d_{\Phi}(C, Q(w))=\mathcal{O}(\ell(w))$ by 5.6.7, we may assume, after conjugating $w$ by a linearly bounded element, that $\bar{C} \cap Q(w) \neq \varnothing$. The proof of 5.10 .9 shows that after conjugating $w$ by a bounded element, there exists a general point in $Q^{*}(w) \cap \bar{C}$. By 5.8.9, $\operatorname{Pc}(w)$ is a standard parabolic subgroup. Suppose similarly that $\operatorname{Pc}(v)$ is a standard parabolic subgroup. Since $v, w$ are conjugate, so are their parabolic closures. After conjugating $w$ by a bounded element, we have $\operatorname{Pc}(v)=\operatorname{Pc}(w)=W_{I}$. Now by 3.1.11, $\left[N\left(W_{I}\right): Z\left(W_{I}\right) W_{I}\right]<\infty$. Hence, after once more conjugating $w$ by a bounded element, we may assume that $v$ and $w$ are conjugate in $W_{I}$. We have reduced the theorem to the case where $\operatorname{Pc}(v)=\operatorname{Pc}(w)=W$ and $W$ irreducible. 
If $W$ is finite, the result is trivial. If $W$ is affine, the result is a special case of 6.4.6. Thus, let us suppose that $W$ is infinite and non-affine. As in the beginning of this proof, conjugate $v$ and $w$ by linearly bounded elements such that there are elements $x \in \bar{C} \cap Q(w), z \in \bar{C} \cap Q(v)$. Let $g \in W$ be such that $g v g^{-1}=w$. Let $\alpha$ be any odd root separating $x$ from $w x$. After multiplying $g$ at the left by a suitable power of $w$, the root $\alpha$ also separates $y:=g z$ from $w y$. By 6.4.2, it follows that for this choice of $g$, we have $\ell(g)=d_{\Phi}(z, g z)+\mathcal{O}(1)=d_{\Phi}(x, y)+\mathcal{O}(1)=\mathcal{O}(\ell(w))$.

6.5. The greatest eigenvalue. In this section we suppose $W$ to be irreducible, infinite, non-affine, $E$ non-degenerate, $\operatorname{Span}(\Pi)=E$, and we fix $w \in W$ such that $\operatorname{Pc}(w)=W$.

We will show in 6.5 .14 that there exists $\lambda>1$ such that $\lambda, \lambda^{-1}$ are simple eigenvalues of $w$ and such that $\lambda^{-1} \leq|\mu| \leq \lambda$ for each (complex) eigenvalue $\mu$ of $w$. Moreover, $U^{0}$ meets the sum of the two corresponding (one-dimensional) eigenspaces. The intersection, denoted by $Q_{0}(w)$, is analogous to the axis of a hyperbolic element of $\mathrm{O}^{+}(n, 1)$.

By the theorem of Perron-Frobenius, if an element of $\operatorname{GL}(E)$ maps a proper cone into its interior, then it has a simple real eigenvalue bounding every complex eigenvalue in absolute value. I have been looking for such a proper cone mapped by $w$ into itself but did not find one. This does not seem to be the right way for showing the desired results. A more direct approach has proved more successful, and it will be given in this section. The most important ingredients are $6.3 .8\left(\left(\alpha, w^{n} \beta\right) \rightarrow \infty\right.$ as $|n| \rightarrow \infty$ whenever $\alpha, \beta$ are outward roots), 6.3.11 (the number of periodic roots is bounded), and the combinatorially flavoured result 6.8 .1 of Section 6.8. A 'disadvantage' of our technique is that we still do not know whether there exists an eigenvalue $\mu \neq \lambda$ with $|\mu|=\lambda$. Conjecturally (6.5.16), such a $\mu$ does not exist.

We use the following notation. We will write $\lambda$ for the maximum of the absolute values of the complex eigenvalues of $w$. We denote the generalized eigenspace of $w$ in $E^{*}$ with eigenvalue $\mu$ by $E_{\mu}$; it is defined by $E_{\mu}=\left\{x \in E^{*} \mid(w-\mu)^{k} x=0\right\}$ where $k$ is big enough, for example, $k=\operatorname{dim} E^{*}$. We write $E_{w}=E_{\lambda}+E_{\lambda-1}$. The projections on $E_{\mu}$ and $E_{w}$ are denoted by $p_{\mu}$ and $p_{w}$, respectively.

We summarize this section. In 6.5.4, it is proved that $\lambda>1, \lambda$ is an eigenvalue of $w$, and $(w-\mu) E_{\mu}=0$ whenever $|\mu|=\lambda$. Theorem 6.5.10 says that $p_{w}(U-\{0\})=$ $U^{0} \cap E_{w}$. In 6.5.14 we show $\operatorname{dim} E_{\lambda}=\operatorname{dim} E_{\lambda-1}=1$.

6.5.1 Definition. For $A \in \mathrm{GL}_{n}(\mathbb{C})$, define $\rho(A)$ to be the maximum of the absolute values of the eigenvalues of $A$. For $\mu \in \mathbb{C}$, define $\operatorname{deg}(\mu, A)$ to be the maximum among the sizes of the Jordan blocks of $A$ with eigenvalue $\mu$ (and 0 if $\mu$ is not an eigenvalue). Equivalently, $\operatorname{deg}(\mu, A)$ is the least $k \geq 0$ such that $(A-\mu)^{k} x=0$ whenever $(A-\mu)^{k+1} x=0$. We define $\operatorname{deg}(A)=\max \{\operatorname{deg}(\mu)|\mu \in \mathbb{C},| \mu \mid=$ $\rho(A)\}$. 
We will make use of similar notations for sequences $a_{0}, a_{1}, \ldots$ of complex numbers of the form

$$
a_{n}=\sum_{\mu \in \mathbb{C}^{*}} c_{\mu}(n) \mu^{n},
$$

where $c_{\mu} \in \mathbb{C}[X]$ are polynomials and almost all $c_{\mu}$ are identically zero, so that the above sum is a finite sum. Such sequences are always considered in the range $n \in \mathbb{N}$ rather than $\mathbb{Z}$ unless stated otherwise.

We call $\mu$ an eigenvalue of the sequence if $c_{\mu} \neq 0$. Define $\rho\left(n \mapsto a_{n}\right)$, or simply $\rho\left(a_{n}\right)$, to be the maximum of the absolute values of the eigenvalues of $a_{n}$. We define $\operatorname{deg}\left(\mu, n \mapsto a_{n}\right)$, or simply $\operatorname{deg}(\mu)$, to be the degree of $c_{\mu}$ plus 1 (the degree of the zero polynomial being -1$)$. Finally, $\operatorname{deg}\left(a_{n}\right)=\max \left\{\operatorname{deg}\left(\mu, n \mapsto a_{n}\right) \mid \mu \epsilon\right.$ $\left.\mathbb{C},|\mu|=\rho\left(a_{n}\right)\right\}$.

6.5.2 Lemma. Let $\left(a_{n}\right)_{n}$ be a non-zero sequence of the form (13). Then there is a unique pair $(\mu, d) \in \mathbb{R}_{>0} \times \mathbb{Z}_{>0}$ such that

$$
\limsup _{n \rightarrow \infty} \frac{\left|a_{n}\right|}{n^{d-1} \mu^{n}} \in \mathbb{R}_{>0} .
$$

It is $\mu=\rho\left(a_{n}\right)$ and $d=\operatorname{deg}\left(a_{n}\right)$.

Proof. Unicity is easy and left to the reader.

Existence. Let $\mu=\rho\left(a_{n}\right), d=\operatorname{deg}\left(a_{n}\right)$. It is easy and left to the reader to show that the limsup is not infinite. We must show that the limsup is non-zero.

Let $\mu_{0} \in \mathbb{C}^{*}$ be such that $\left|\mu_{0}\right|=\mu$ and $\operatorname{deg}\left(\mu_{0}\right)=d$. Choose $p_{0}, \ldots, p_{k} \in \mathbb{C}$ such that the sequence $b_{n}$ defined by

$$
b_{n}=p_{0} a_{n}+p_{1} a_{n+1}+\cdots+p_{k} a_{n+k}
$$

has no eigenvalues $v$ with $|v|=\mu, v \neq \mu_{0}$, and such that $\operatorname{deg}\left(\mu_{0}, b_{n}\right)=d$. It is now easy to see that

$$
\limsup _{n \rightarrow \infty} \frac{\left|b_{n}\right|}{n^{d-1} \mu^{n}} \neq 0 .
$$

It follows that the limsup with $a_{n}$ is non-zero too.

6.5.3 Lemma. Let $\left(a_{n}\right)_{n}$ be a sequence of the form (13). Let $\mu=\rho\left(a_{n}\right)$ and $d=$ $\operatorname{deg}\left(a_{n}\right)$. If $a_{n}>0$ for all $n$, then $d=\operatorname{deg}(\mu)$.

Proof. Recall that $P[X]$ denotes the set of nonzero polynomials in $\mathbb{R}[X]$ whose coefficients are nonnegative. By 5.6.8, there exists $f \in P[X]$ such that for every eigenvalue $\lambda \notin \mathbb{R}_{>0}$, the polynomial $(X-\lambda)^{\operatorname{deg}\left(c_{\lambda}\right)+1}$ divides $f$. Write $f=p_{0}+$ $p_{1} X+\cdots+p_{k} X^{k}$. Then the sequence $\left(b_{n}\right)_{n}$ defined by

$$
b_{n}=p_{0} a_{n}+p_{1} a_{n+1}+\cdots+p_{k} a_{n+k}
$$


has only eigenvalues in $\mathbb{R}_{>0}$. Since all $a_{n}, p_{i} \geq 0$, we have

$$
\limsup _{n \rightarrow \infty} \frac{\left|b_{n}\right|}{n^{d-1} \mu^{n}} \in \mathbb{R}_{>0} .
$$

by the existence part of 6.5.2. Since all eigenvalues of $b_{n}$ are in $\mathbb{R}_{>0}$, it follows by the unicity part of 6.5.2 that $d=\operatorname{deg}\left(\mu, b_{n}\right)$, whence $d=\operatorname{deg}\left(\mu, a_{n}\right)$.

6.5.4 Lemma. (a) $\lambda>1$.

(b) Let $\alpha, \beta$ be two outward roots. Then $\lambda$ is an eigenvalue of the sequence $\left(\alpha, w^{n} \beta\right)$. In particular, $\lambda$ is an eigenvalue of $w$.

(c) $\operatorname{deg}(w)=1$.

Proof. (a) Choose any outward root $\alpha$. By 6.3.7, there exists $m>0$ such that $\left(\alpha, w^{m} \alpha\right)>1$, say $\left(\alpha, w^{m} \alpha\right)=\left(\mu+\mu^{-1}\right) / 2, \mu>1$. By applying 6.2.2 inductively to the sequences $\alpha<w^{n m} \alpha<w^{(n+1) m} \alpha$, we find $\left(\alpha, w^{n m} \alpha\right) \geq\left(\mu^{n}+\mu^{-n}\right) / 2$. This shows that $\rho(w) \geq \mu^{1 / m}>1$.

(b) For outward roots $\alpha, \beta$, write

$$
\lambda_{\alpha \beta}=\rho\left(n \mapsto\left(\alpha, w^{n} \beta\right)\right), \quad d_{\alpha \beta}=\operatorname{deg}\left(n \mapsto\left(\alpha, w^{n} \beta\right)\right) .
$$

First, we will show that $\lambda_{\alpha \beta}$ and $d_{\alpha \beta}$ do not depend on $\alpha, \beta$. Let $\alpha, \beta, \alpha^{\prime}, \beta^{\prime}$ be outward. Then there are $k, \ell \in \mathbb{Z}$ such that $\alpha<w^{k} \alpha^{\prime}$ and $w^{\ell} \beta^{\prime}<\beta$ by 6.3.9. For big $n$, we have

$$
\alpha<w^{k} \alpha^{\prime}<w^{\ell+n} \beta^{\prime}<w^{n} \beta .
$$

By applying 6.2.3 twice, we have

$$
\left(\alpha, w^{n} \beta\right) \geq\left(w^{k} \alpha^{\prime}, w^{\ell+n} \beta^{\prime}\right)=\left(\alpha^{\prime}, w^{\ell+n-k} \beta^{\prime}\right) .
$$

By 6.3.8 we may write for big $n$

$$
\left(\alpha, w^{n} \beta\right)=\left(a_{n}+a_{n}^{-1}\right) / 2, \quad\left(\alpha^{\prime}, w^{n} \beta^{\prime}\right)=\left(b_{n}+b_{n}^{-1}\right) / 2, \quad a_{n}, b_{n}>1 .
$$

By (14) we have

$$
a_{n} \geq b_{\ell+n-k} .
$$

By 6.5.2, it follows that $\lambda_{\alpha \beta} \geq \lambda_{\alpha^{\prime} \beta^{\prime}}$, and, by symmetry, all $\lambda_{\alpha \beta}$ are equal. By combining (15) and 6.5.2 again, it follows that all $d_{\alpha \beta}$ are equal.

Since $E$ is non-degenerate and the outward roots span $E$, there exist $\alpha, \beta$ with $\lambda_{\alpha \beta}=\lambda$. Since $\left(\alpha, w^{n} \beta\right)>0$ for $n$ big, 6.5.3 implies that $\lambda$ is an eigenvalue of the sequence $\left(\alpha, w^{n} \beta\right)$.

(c) Let $\alpha$ be an outward root. By 6.3.8, almost all $n \in \mathbb{N}$ satisfy $\left(\alpha, w^{n} \alpha\right)>1$. It follows that almost all $n \in \mathbb{N}$ satisfy $\left(\alpha, w^{n k} \alpha\right)>1$ for all $k>0$; let $L$ be the set of such $n$. 
For $n \in L$, define $c_{n}$ by $\left(\alpha, w^{n} \alpha\right)=\left(c_{n}+c_{n}^{-1}\right) / 2, c_{n}>1$. As in the proof of (a), one finds that $\left(\alpha, w^{n k} \alpha\right) \geq\left(c_{n}^{k}+c_{n}^{-k}\right) / 2$ for all $k \geq 1$. On writing $d=\operatorname{deg}(w)$, we have

$$
\limsup _{k \rightarrow \infty} \frac{c_{n}^{k}}{2(n k)^{d-1} \lambda^{n k}} \leq \limsup _{k \rightarrow \infty} \frac{\left|\left(\alpha, w^{n k} \alpha\right)\right|}{(n k)^{d-1} \lambda^{n k}} \leq \limsup _{n \rightarrow \infty} \frac{\left|\left(\alpha, w^{n} \alpha\right)\right|}{n^{d-1} \lambda^{n}}<\infty .
$$

Hence $c_{n} \leq \lambda^{n}$ for all $n \in L$. It follows that

$$
\limsup _{n \rightarrow \infty} \frac{\left|\left(\alpha, w^{n} \alpha\right)\right|}{n \lambda^{n}} \leq \limsup _{n \rightarrow \infty} \frac{\lambda^{n}+\lambda^{-n}}{2 n \lambda^{n}}=0,
$$

whence $d_{\alpha \alpha}=1$ by 6.5.3. Since all $d_{\alpha \beta}$ are equal, it follows that $\operatorname{deg}(w)=1$, which finishes the proof of (c).

In words, 6.5.4(c) says that the generalized eigenspace $E_{\mu}$ in $E^{*}$ of $w$ with eigenvalue $\mu$ is in fact the eigenspace, if $|\mu|=\lambda$. The rest of this section is devoted to proving that $\operatorname{dim} E_{\lambda}=1$.

6.5.5 Lemma. Let $A \in \mathrm{O}(k, \ell)$, and let $E_{\mu}$ denote the generalized eigenspace of $A$ with eigenvalue $\mu$. Then the following holds.

(a) If $\mu \nu \neq 1$ then $E_{\mu} \perp E_{v}$.

(b) The bilinear form $(\cdot, \cdot)$ defines a pairing $E_{\mu} \times E_{\mu^{-1}} \rightarrow \mathbb{R}$ if $\mu^{2} \neq 1$.

(c) For every $\mu \in \mathbb{C}^{*}$, the vector spaces $E_{\mu}$ and $E_{\mu^{-1}}$ have equal dimensions.

Proof. (a) Let $x \in E_{\mu}, y \in E_{v}$ be a counterexample, such that $k+\ell$ is minimal, where $(A-\mu)^{k} x=(A-v)^{\ell} y=0$. Then, using minimality,

$$
\begin{aligned}
(x, y) & =(A x, A y)=((A x-\mu x)+\mu x, A y) \\
& =\mu(x, A y)=\mu(x,(A y-v y)+v y)=\mu \nu(x, y),
\end{aligned}
$$

whence $(x, y)=0$. This is the desired contradiction. Part (b) follows from (a) and the fact that $(\cdot, \cdot)$ is non-degenerate. Part (c) is an immediate consequence of (b).

By 6.5.5(c), $E_{\mu}$ and $E_{\mu^{-1}}$ have equal dimensions for all $\mu$. Hence $\lambda^{-1}$ is an eigenvalue of $w$, and every eigenvalue $\mu$ satisfies $\lambda^{-1} \leq|\mu| \leq \lambda$.

6.5.6 Lemma. Let $x \in U-\{0\}$ and let $\alpha$ be an outward root. Then $\lambda$ is an eigenvalue of the sequence $\left\langle x, w^{n} \alpha\right\rangle$.

Proof. Since $x \neq 0$ there exists an outward root $\beta$ such that $\langle x, \beta\rangle \neq 0$. For $k>0$ big enough we have $\left\langle x, w^{k} \beta\right\rangle \geq 0$. We cannot have equality for all big $k>0$ because this would contradict $\langle x, \beta\rangle \neq 0$. Hence there exists $k$ such that $\left\langle x, w^{k} \beta\right\rangle>0$. For 
big $n$, we have $w^{k} \beta<w^{n} \alpha$ by 6.3.9, say $\left(w^{k} \beta, w^{n} \alpha\right)=\left(a_{n}+a_{n}^{-1}\right) / 2, a_{n}>1$. By 1.4.7(c), we find that

$$
\left\langle x, w^{n} \alpha\right\rangle \geq a_{n}\left\langle x, w^{k} \beta\right\rangle .
$$

From 6.5.4(b), it follows that $\rho\left(n \mapsto\left\langle x, w^{n} \alpha\right\rangle\right)=\rho\left(n \mapsto a_{n}\right)=\lambda$. Since $\left\langle x, w^{n} \alpha\right\rangle>0$ for $n$ big enough, 6.5.3 implies that $\lambda$ is an eigenvalue of the sequence $\left\langle x, w^{n} \alpha\right\rangle$.

6.5.7 Lemma. For all $x \in U-\{0\}$, the points $p_{\lambda}(x)$ and $p_{\lambda-1}(x)$ are non-zero.

Proof. This follows immediately from 6.5.6, applied to $w$ and $w^{-1}$.

6.5.8 Lemma. On writing $r=\# S$, we have

$$
\left(1+w+\cdots+w^{r-1}\right)(U-\{0\}) \subset U^{0} .
$$

Proof. Let $x \in U-\{0\}$. Let $H=\left(w^{\mathbb{Z}} x\right)^{\prime}$. Then $H$ is a parabolic subgroup, normalized by $w$. Since $\operatorname{Pc}(w)=W$, we find $\operatorname{Pc}(N(H))=W$. By 6.8.1, either $H$ is finite or $H=W$. In the latter case, $x=0$ because $E$ is spanned by $\Pi$, a contradiction. Hence $H$ is finite. Note that $H=\left\{x, w x, \ldots, w^{r-1} x\right\}^{\prime}$. By 2.1.2, the cone $X$ spanned by $\left\{x, w x, \ldots, w^{r-1} x\right\}$ contains a point $y$ such that $y^{\prime}=H$. By 2.2.5, $y \in U^{0}$. By multiplying $y$ by a scalar and adding another point of $X$, we find that $\left(1+w+\cdots+w^{r-1}\right) x \in U^{0}$.

We recall the notation $E_{>0}$ for the sum of the generalized eigenspaces in $E^{*}$ of $w$ with positive real eigenvalues. Since $\operatorname{deg}(w)=1$ by $6.5 .4(\mathrm{c})$, we have $p_{\lambda}(x)=$ $\lim _{n \rightarrow \infty} w^{n} x / \lambda^{n}$ for all $x \in E_{>0}$. Applying this result to $w^{-1}$ as well, we find that

$$
p_{w}(x)=\lim _{n \rightarrow \infty}\left(w^{n} x+w^{-n} x\right) / \lambda^{n} \quad \text { for all } x \in E_{>0} .
$$

6.5.9 Lemma. We have $p_{w}\left(Q_{1}(w)\right) \subset U-\{0\}$.

Proof. Let $x \in Q_{1}(w)$. Write $x_{n}=w^{n} x+w^{-n} x$. Since $x \in Q_{1}(w) \subset E_{>0}$, we have (16) $p_{w}(x)=\lim _{n \rightarrow \infty} x_{n} / \lambda^{n}$. The proof will be finished if we show that $d_{\Phi}\left(x, x_{n}\right)$ is bounded as $n \rightarrow \infty$, since then $\left\{x_{1}, x_{2}, \ldots\right\}$ is contained in the union $X$ of finitely many facets, whence $p_{w}(x) \in \bar{X} \subset U$, and $p_{w}(x) \neq 0$ by 6.5.7.

Since $Q_{1}(w)$ is convex by 5.6.2, we have $x_{n} \in Q_{1}(w)$ for all $n$. A root $\alpha$ separating $x$ from $x_{n}$ is never even, because if $\alpha$ were even and, say, supporting, then $x, x_{n} \in Q_{1}(w) \subset Q(w) \subset H(\alpha)$. There are only finitely many periodic roots, by 6.3.11, so we need only consider odd roots. (In fact, periodic roots do not separate $x$ from $x_{n}$, but we do not need this.) Let $\alpha$ be outward. Since $x \in E_{>0}$ and $\operatorname{deg}(w)=1$ (6.5.4 (c)), there exist $a, b, M, \mu, \varepsilon_{k} \in \mathbb{R}$ such that $1<\mu<\lambda$, and for all $k \in \mathbb{Z}$,

$$
\left\langle x, w^{k} \alpha\right\rangle=a \lambda^{k}-b \lambda^{-k}+\varepsilon_{k} \mu^{|k|}, \quad\left|\varepsilon_{k}\right| \leq M .
$$


We will show that if $w^{k} \alpha$ separates $x$ from $x_{n}$, then

$$
\left|a \lambda^{k}-b \lambda^{-k}\right| \leq 2 M \mu^{|k|} .
$$

We have $x \in Q(w) \subset U^{0}$, so $x \neq 0$. By 6.5.7, $a, b \neq 0$. Since $\alpha$ is outward, we have $a, b>0$. We have

$$
\begin{aligned}
\left\langle x_{n}, w^{k} \alpha\right\rangle= & \left\langle w^{n} x+w^{-n} x, w^{k} \alpha\right\rangle \\
= & \left\langle x, w^{k+n} \alpha+w^{k-n} \alpha\right\rangle \\
= & \left(a \lambda^{k+n}-b \lambda^{-k-n}+\varepsilon_{k+n} \mu^{|k+n|}\right) \\
& \quad+\left(a \lambda^{k-n}-b \lambda^{-k+n}+\varepsilon_{k-n} \mu^{|k-n|}\right) \\
= & \left(\lambda^{n}+\lambda^{-n}\right)\left(a \lambda^{k}-b \lambda^{-k}\right)+2 \varepsilon \mu^{|n|+|k|}, \quad|\varepsilon| \leq M .
\end{aligned}
$$

If $w^{k} \alpha$ separates $x$ from $x_{n}$, then $\left\langle x, w^{k} \alpha\right\rangle$ or $\left\langle x_{n}, w^{k} \alpha\right\rangle$ has different sign than $a \lambda^{k}-b \lambda^{-k}$. In the first case, from (17) we find

$$
\left|a \lambda^{k}-b \lambda^{-k}\right| \leq M \mu^{|k|},
$$

whereas the latter yields

$$
\left|a \lambda^{k}-b \lambda^{-k}\right| \leq \frac{2 M \mu^{|n|+|k|}}{\lambda^{n}+\lambda^{-n}} \leq 2 M \mu^{|k|}
$$

by (19), and either case implies (18). Since (18) has only finitely many solutions in $k$, the number of roots of the form $w^{k} \alpha$ separating $x$ from $x_{n}$ is bounded as $n \rightarrow \infty$. Since the number of $w^{\mathbb{Z}}$-orbits of odd roots is finite, $d_{\Phi}\left(x, x_{n}\right)$ is bounded as $n \rightarrow \infty$, as promised.

6.5.10 Theorem. We have $p_{w}(U-\{0\})=U^{0} \cap E_{w} \subset Q_{1}(w)$.

Proof. Let $f \in P[X]$ be a polynomial such that all eigenvalues of $f(w)$ are in $\mathbb{R}_{\geq 0}$ (see 5.6.9) and such that $1+X+\cdots+X^{N r-1}$ divides $f$, where $N$ is the constant of 5.6.5. Let $g=f(X) f\left(X^{-1}\right), h=g(X) / g(\lambda)$. Since $h(\lambda)=h\left(\lambda^{-1}\right)=1$ and $\operatorname{deg}(w)=1$, we have $p_{w}=p_{w} \circ h(w)=h(w) \circ p_{w}$. We will show that

$$
h(w)(U-\{0\}) \subset Q_{1}(w) .
$$

Let $x \in U-\{0\}$. Write $h=p q$ where $q=1+X+\cdots+X^{r-1}$. By 6.5.8, we have $q(w) x \in U^{0}$. Moreover, $h(w) x \in Q_{2}(w)$ because $1+X+\cdots+X^{N-1}$ divides $p$ (see 5.6.5). We even have $h(w) x \in Q_{1}(w)$, since all eigenvalues of $h(w)$ are in $\mathbb{R}_{\geq 0}$. This concludes the proof of (20). 
Now we will prove the theorem. The inclusion $p_{w}(U-\{0\}) \supset U^{0} \cap E_{w}$ is trivial. As to the reverse inclusion, we have

$$
\begin{gathered}
p_{w}(U-\{0\})=h(w) \circ p_{w} \circ h(w)(U-\{0\}) \stackrel{(20)}{\subset} h(w) \circ p_{w} Q_{1}(w) \\
\stackrel{6.5 .9}{\subset} h(w)(U-\{0\}) \stackrel{(20)}{\subset} Q_{1}(w) \subset U^{0},
\end{gathered}
$$

and clearly $p_{w}(U) \subset E_{w}$. We have shown that $p_{w}(U-\{0\})=U^{0} \cap E_{w} \subset Q_{1}(w)$.

6.5.11 Definition. We define $Q_{0}(w)=p_{w}(U-\{0\})$. Thus, by 6.5.10, we have $Q_{0}(w)=U^{0} \cap E_{w}=p_{w}\left(U^{0}\right)$ and $Q_{0}(w) \subset Q_{1}(w)$.

Let $C(w)$ denote the conjugacy class of $w$ in $W$.

6.5.12 Lemma. Let $w \in W$ be an element in a Coxeter group. Then there are only finitely many $Z(w)$-orbits of facets in $Q(w)$.

Proof. It is enough to prove that there are only finitely many $Z(w)$-orbits of closed chambers $g \bar{C}$ meeting $Q(w)$. We have a bijection $Z(w) \backslash W \rightarrow C(w), Z(w) g \mapsto$ $g^{-1} w g$. It remains to prove that $\ell\left(g^{-1} w g\right)$ is bounded as long as $g \bar{C}$ meets $Q(w)$. Let $x \in g \bar{C} \cap Q(w)$. By 5.5.7, $d_{\Phi}(x, w x)=r(w)$. So $\ell\left(g^{-1} w g\right)=d_{\Phi}(g C, w g C) \leq$ $d_{\Phi}(g C, x)+d_{\Phi}(x, w x)+d_{\Phi}(w x, w g C)=r(w)+$ a bounded number. Hence $\ell\left(g^{-1} w g\right)$ is bounded.

6.5.13 Lemma. Let $\alpha$ be an outward root. Then $Q(w) \cap K(-\alpha) \cap K(w \alpha)$ is the union of finitely many facets.

Proof. By 6.5.12, there are only finitely many $Z(w)$-orbits of facets in $Q(w)$. Since $\left[Z(w): w^{\mathbb{Z}}\right]<\infty$, there are only finitely many $w^{\mathbb{Z}}$-orbits of facets in $Q(w)$. Each orbit has only a finite number of facets contained in $K(-\alpha) \cap K(w \alpha)$, by definition of outwardness, whence the result.

6.5.14 Theorem. We have $\operatorname{dim} E_{\lambda}=\operatorname{dim} E_{\lambda-1}=1$. There exist non-zero $u_{1} \in$ $E_{\lambda-1}, u_{2} \in E_{\lambda}$ such that $Q_{0}(w)=\mathbb{R}_{>0} u_{1}+\mathbb{R}_{>0} u_{2}$.

Proof. Consider the set $X=p_{\lambda}\left(U^{0}\right)$. Note that $0 \notin X$ by 6.5.7. We will show that $X$ is open and closed in $E_{\lambda}-\{0\}$.

Open. This follows from the fact that $p_{\lambda}: E^{*} \rightarrow E_{\lambda}$ is a surjective linear map and $U^{0}$ is open in $E^{*}$.

Closed. Since $p_{\lambda}=p_{\lambda} p_{w}$, we have

$$
X=p_{\lambda}\left(U^{0}\right)=p_{\lambda} p_{w}\left(U^{0}\right)=p_{\lambda}\left(Q_{0}(w)\right) .
$$


Let $\alpha$ be an outward root. Then every $w$-orbit $w^{\mathbb{Z}} x$, with $x \in U^{0}$, meets $Y:=$ $K(-\alpha) \cap K(w \alpha)$. Hence

$$
X=p_{\lambda}\left(Q_{0}(w)\right)=p_{\lambda}(Z),
$$

where $Z=Q_{0}(w) \cap Y$. By 6.5.13 and the fact that $Q_{0}(w) \subset Q(w)$, the set $Z$ is contained in only finitely many facets. Hence $\bar{Z} \subset U$ and

$$
\begin{aligned}
\bar{X} & =p_{\lambda}(\bar{Z}) \subset p_{\lambda}(U)=p_{\lambda}(U-\{0\}) \cup\{0\} \\
& =p_{\lambda} p_{w}(U-\{0\}) \cup\{0\} \subset p_{\lambda}\left(U^{0}\right) \cup\{0\}=X \cup\{0\},
\end{aligned}
$$

where in the last $\subset$ we use 6.5.10. Hence $X$ is closed in $E_{\lambda}-\{0\}$.

Suppose that $\operatorname{dim} E_{\lambda}>1$. Then $E_{\lambda}-\{0\}$ is connected. Since $X \subset E_{\lambda}-\{0\}$ is nonempty, open and closed, we have $X=E_{\lambda}-\{0\}$. But this contradicts the fact that $X$ is closed under addition. Hence $\operatorname{dim} E_{\lambda}=1$. Since $X$ is a non-empty cone, there exists $u_{2} \in E_{\lambda}-\{0\}$ such that $X=\mathbb{R}_{>0} u_{2}$. Similarly, $p_{\lambda-1}\left(U^{0}\right)=\mathbb{R}_{>0} u_{1}$ for some $u_{1}$. We have $Q_{0}(w)=p_{w}\left(U^{0}\right)=\left(p_{\lambda}+p_{\lambda-1}\right) U^{0} \subset p_{\lambda}\left(U^{0}\right)+p_{\lambda-1}\left(U^{0}\right)=\mathbb{R}_{>0} u_{1}+$ $\mathbb{R}_{>0} u_{2}$. So $Q_{0}(w)$ is a non-empty $w$-invariant convex subset of $\mathbb{R}_{>0} u_{1}+\mathbb{R}_{>0} u_{2}$, that is, the whole of $\mathbb{R}_{>0} u_{1}+\mathbb{R}_{>0} u_{2}$.

6.5.15 Proposition. Let $\alpha$ be a root. Then $Q_{0}(w) \subset \mu(\alpha)$ if and only if $\alpha$ is periodic.

Proof. 'If'. Let $\alpha$ be periodic, and let $x \in Q_{0}(w)$. Then the sequence $\left\langle w^{n} x, \alpha\right\rangle$ has only eigenvalues $\lambda$ and $\lambda^{-1}$. But this sequence equals $\left\langle x, w^{-n} \alpha\right\rangle$ and hence has only roots of unity as eigenvalues, since $\alpha$ is periodic. Since $\lambda>1$, we find that $\langle x, \alpha\rangle=0$.

'Only if'. Choose any $x \in Q_{0}(w)$. Then all $\mu\left(w^{n} \alpha\right)$ contain $x$. Since there are only finitely many walls through $x$, the root $\alpha$ is periodic.

By definition of $\lambda$, for every eigenvalue $\mu$ of $w$, we have $|\mu| \leq \lambda$. The following conjecture sharpens this.

6.5.16 Conjecture. For every eigenvalue $\mu$ of $w$ different from $\lambda$, we have $|\mu|<\lambda$.

6.6. Removed. This section has been removed from the thesis, but its number is retained to ease reference.

6.7. Second solution to the conjugacy problem. In this section, unless stated otherwise, $W$ denotes an infinite, irreducible, non-affine Coxeter group, and $(E,(\cdot, \cdot), \Pi)$ an associated non-degenerate root basis with $\operatorname{Span} \Pi=E$.

We suppose that the root basis is defined over a real number field $K$ (see 4.7.5).

We retain the notations of Section 6.5. Thus, if some $w \in W$ with $\operatorname{Pc}(w)=W$ has been chosen, we write $\lambda=\rho(w)$ and $Q_{0}(w)=\mathbb{R}_{>0} u_{1}+\mathbb{R}_{>0} u_{2}$, where $w u_{1}=$ $\lambda^{-1} u_{1}, w u_{2}=\lambda u_{2}$. 
By 6.5.15, $Q_{0}(w)$ is contained in a wall $\mu(\alpha)$ if and only if $\alpha$ is periodic. Call a point $x \in Q_{0}(w)$ special if it is on at least one non-periodic (necessarily odd) wall. Let $x_{k}=a_{k} u_{1}+b_{k} u_{2}(k \in \mathbb{Z})$ denote the special points on $Q_{0}(w)$ up to multiples, ordered in the sense that $b_{k} / a_{k}$ is strictly increasing. There exists $m=m(w) \leq \ell(w)$ such that, after replacing $x_{k}$ by multiples if necessary, $w x_{k}=x_{k+m}$.

For every $g \in W$, the cone $Q_{0}(w) \cap g \bar{C}$ is the convex span of two, one or no consecutive $x_{k}$, that is, it is $\mathbb{R}_{>0} x_{k}+\mathbb{R}_{>0} x_{k+1}$ or $\mathbb{R}_{>0} x_{k}$ or $\varnothing$ for some $k$.

6.7.1 Definition. We define $M_{0}(w)$ to be the set of conjugates $g^{-1} w g$ to $w$ such that $Q_{0}(w) \cap g \bar{C}$ has dimension 2 .

6.7.2 Proposition. There exists a quadratic algorithm that, when given $w \in W$ with $\operatorname{Pc}(w)=W$, decides whether $w \in M_{0}(w)$.

Proof. Let $u_{1}, u_{2}, \lambda$ be as above. It has to be decided whether $Q_{0}(w) \cap \bar{C}$ has dimension 2, that is, whether there exist at least two $t \in \mathbb{R}_{>0}$ such that $\left\langle u_{1}+t u_{2}, e_{s}\right\rangle \geq$ 0 for all $s \in S$. This is equivalent to a fixed number of inequalities $g_{i}(\lambda)>0$, where $g_{1}, \ldots, g_{n} \in K[X]$ have heights $\mathcal{O}(\ell(w))$ and bounded degrees. By 5.9.2, this can be tested in quadratic time (take $f$ to be the characteristic polynomial of $w$ ).

6.7.3 Proposition. There exists a cubic algorithm that, given $w \in W$ with $\operatorname{Pc}(w)=$ $W$, computes an element of $M_{0}(w)$.

Proof. The algorithm runs as follows. In cubic time, compute a point $x \in Q(w)$ of depth $\mathcal{O}(\ell(w))$ by 5.10.6. Conjugate $w$ so as to get $x \in \bar{C}$. By 5.10.5, we can compute the canonical outward roots in cubic time. Let $\alpha$ be a canonical outward root. Note that $Q_{0}(w) \cap \mu(\alpha)=\mathbb{R}_{>0} y$ for some $y \in U^{0}$. Let $y_{1}, \ldots, y_{k}$ be the coordinates of $y$ with respect to a basis of $E^{*}$ that was fixed in advance. We may suppose that $y_{i}=1$ for some $i$. Let $f$ denote the characteristic polynomial of $w$ and $\lambda$ its greatest real root. Now every coordinate $y_{i}$ equals a polynomial (depending on $y_{i}$ ) over $K$ in $\lambda$, of degree $\leq k$ and height $\mathcal{O}(\ell(w))$. Moreover, $d_{\Phi}(y, C)=\mathcal{O}(\ell(w))$ by 6.4.2. It follows that the algorithm 1.2.4, applied to $y$, is cubic (each step is quadratic by 5.9.2 and the number of steps is linear - compare 5.10.4). This algorithm computes $g \in W$ such that $y \in g \bar{C}$. By conjugating $w$, we may suppose that $y \in Q_{0}(w) \cap \bar{C}$. Determine $I \subset S$ such that $y \in C_{I}$. Then for some $h \in W_{I}$, we have $h^{-1} w h \in M_{0}(w)$. But $W_{I}$ is finite. By simply testing all $h \in W_{I}$ (in quadratic time by 6.7.2), we find an element of $M_{0}(w)$.

6.7.4 Theorem. For every (finite rank) Coxeter group, there is a cubic solution to the conjugacy problem.

Proof. In a way similar to 6.4.4, the problem is reduced to the case $\operatorname{Pc}(v)=\operatorname{Pc}(w)=$ $W$ is irreducible, infinite, non-affine. The algorithm continues as follows. By 6.7.3, 
compute $g \in W$ such that $g^{-1} w g \in M_{0}(w)$, that is, $g \bar{C} \cap Q_{0}(w)=\mathbb{R}_{>0} x_{0}+\mathbb{R}_{>0} x_{1}$, after renumbering the $x_{i}$ if necessary. Thus we have computed two consecutive special points $x_{0}, x_{1}$ in $Q_{0}(w)$, that is, we have expressed their coordinates as polynomials (of height $\mathcal{O}(\ell(w))$ and bounded degree) over $K$ in $\lambda$. We compute (in the same sense) $x_{2}, x_{3}, \ldots$ as follows. Suppose that $x_{n}$ has been computed. Compute all $g \in W$ such that $x_{n} \in g \bar{C}$. At least one of these $g$ has the property $g \bar{C}=\mathbb{R}_{>0} x_{n}+\mathbb{R}_{>0} y$ for some $y$ independent of $x_{n}$ and independent of $x_{n-1}$. Then $x_{n+1}$ may be chosen to be $y$. Computing each next $x_{n}$ takes quadratic time. After computing each new $x_{n}$, check whether $x_{n} \in \mathbb{R}_{>0} x_{0}$. If so, we have $n=m(w)$, and we stop computing any more $x_{i}$. Now it is easy to compute all of $M_{0}(w)$, in $\mathcal{O}\left(m(w) \ell(w)^{2}\right)=$ cubic time. The algorithm is finished by computing one element $x$ of $M_{0}(v)$, and checking whether it is in $M_{0}(w)$. This can also be done in cubic time because $x$ must be compared to $\# M_{0}(w)=\mathcal{O}(\ell(w))$ elements, and each comparison takes quadratic time.

6.7.5 Remark. Let $W \subset \mathrm{O}^{+}(n, 1)$ be a hyperbolic reflection group. The conjugacy problem for hyperbolic elements in $W$ can be solved by the ideas of this section. The only point of difference may be 6.7.3.

To explain this, let us denote by $L(w)$ the analogue to $Q_{0}(w)$, that is, $L(w)=$ $\mathbb{R}_{>0} u_{1}+\mathbb{R}_{>0} u_{2}$, where $u_{1}, u_{2}$ are eigenvectors of $w$ representing the fixed points at infinity. The analogue of 6.7.3 should compute $g \in W$ such that $g \bar{D} \cap L(w) \neq \varnothing$. Such a $g$ is found by choosing some $y \in L(w)$ and then computing $g \in W$ such that $y \in g \bar{D}$. The algorithm is polynomial if the $\operatorname{depth} \operatorname{dp}(y)$ (see page 130) is polynomial in $\ell(w)$.

One choice of $y$ could be the projection on $L(w)$ of a point $x \in D$, fixed in advance. We conjecture that $\operatorname{dp}(y)$ is $\mathcal{O}(\ell(w))$ then. I have not proved anything in this direction. Another choice is that $\mathbb{R} y$ is the intersection of $L(w)$ with an odd wall (see Section 5).

Using results of Sections 5 and 6, it can then be proved that $\operatorname{dp}(y)$ is $\mathcal{O}(\ell(w))$, analogous to 6.4.2.

6.8. Free abelian groups in Coxeter groups. The algebraic rank of a group $G$ is defined to be the supremum of the ranks of the free abelian subgroups of $G$ and is denoted $\operatorname{rk}(G)$. For Coxeter systems $(W, S)$, this should not be confused with the rank \#S. In this section we will compute the algebraic rank of a Coxeter group in terms of the Coxeter matrix.

6.8.1 Lemma. Suppose that $W$ is irreducible. Let $I \subset S$ be such that the standard parabolic closure of $N\left(W_{I}\right)$ equals $W$. Then $I$ is spherical or $I=S$.

Proof. Suppose $I$ to be non-spherical. Let $J$ be a non-spherical component of $I$. By 3.1.9, we have $N\left(W_{I}\right)=G_{I} \ltimes W_{I}$. Let $g \in G_{I}$. Then $g^{-1} \Pi_{J}=\Pi_{K}$ for some 
$K \subset I$. By 3.1.3 there exists a directed path

$$
J=I_{0} \stackrel{s_{0}}{\rightarrow} I_{1} \stackrel{s_{1}}{\rightarrow} \cdots \stackrel{s_{t}}{\rightarrow} I_{t+1}=K
$$

in $K$ such that

$$
g=v\left(I_{0}, s_{0}\right) \ldots v\left(I_{t}, s_{t}\right) .
$$

The existence of $v\left(I_{0}, s_{0}\right)$ implies that $I_{0} \cup\left\{s_{0}\right\}$ contains a spherical component. Since $I_{0}=J$ is infinite and irreducible, we must have $s_{0} \in J^{\perp}$. Hence $I_{1}=J$ and $v\left(I_{0}, s_{0}\right)=s_{0}$. Going on this way, we find $g \in W_{J \perp}$. We conclude that $G_{I} \subset W_{J \perp}$ and $N\left(W_{I}\right) \subset W_{I \cup J \perp}$. Since the standard parabolic closure of $N\left(W_{I}\right)$ equals $W$ and is contained in $W_{I \cup J \perp}$, we find $I \cup J^{\perp}=S$. But $J$ is a component of $I \cup J^{\perp}$ so $J=S$. Also $J \subset I$ so $I=S$.

6.8.2 Theorem. Let $W$ be an irreducible, infinite, non-affine Coxeter group and let $H \subset W$ be an abelian subgroup with $\operatorname{Pc}(H)=W$. Then $H$ does not contain $a$ subgroup isomorphic to $\mathbb{Z}^{2}$.

Proof. Suppose that $A \subset H$ is a subgroup isomorphic to $\mathbb{Z}^{2}$. Let $h \in A-\{1\}$. We may suppose $\operatorname{Pc}(h)$ to be standard parabolic, say $\operatorname{Pc}(h)=W_{I}$. Then

$$
H \subset N\left(W_{I}\right)
$$

since for all $g \in H: g W_{I} g^{-1}=g \operatorname{Pc}(h) g^{-1}=\operatorname{Pc}\left(g h g^{-1}\right)=\operatorname{Pc}(h)=W_{I}$. From (21) and $\operatorname{Pc}(H)=W$ it follows that $W=\operatorname{Pc}\left(N\left(W_{I}\right)\right)$. By 6.8.1, we have $I=S$ (note that $I$ is not spherical because $h \in W_{I}$ has infinite order). Hence $\operatorname{Pc}(h)=W$. This allows us to apply 6.3.10, which states $\left[Z(h): h^{\mathbb{Z}}\right]<\infty$. But $\mathbb{Z}^{2} \cong A \subset Z(h)$. This contradiction finishes the proof.

For the purpose of fixing an unclarity in the proof of 6.8.3, the statement of 6.8.2 is slightly different from the one in the original thesis, which stated the following. Let $W$ be an irreducible, infinite, non-affine Coxeter group and let $H \subset W$ be a free abelian subgroup with $\operatorname{Pc}(H)=W$. Then $H$ is infinite cyclic.

Let $I_{1}, \ldots, I_{n} \subset S$ be irreducible, non-spherical and pairwise perpendicular. For all $i$, let $H_{i} \subset W_{I_{i}}$ be a subgroup as follows. If $I_{i}$ is affine, $H_{i}$ is the translation subgroup. Otherwise, $H_{i}$ is any subgroup of $W_{I_{i}}$ isomorphic to $\mathbb{Z}$. We call $\prod_{i} H_{i}$ a standard free abelian subgroup.

6.8.3 Theorem. Let $W$ be a Coxeter group (of finite rank). Then each free abelian subgroup of $W$ has a finite index subgroup which is conjugate to a subgroup of a standard free abelian subgroup.

Proof. Since standard free abelian subgroups of $W$ have bounded rank, it is enough to show that every free abelian subgroup of finite rank verifies the theorem. Let $G \subset W$ be free abelian of finite rank. 
After conjugating $G$ if necessary, we may suppose $\operatorname{Pc}(G)$ to be standard parabolic, say $\operatorname{Pc}(G)=W_{I}$. Let $I_{1}, \ldots, I_{n}$ be the components of $I$. Let $G_{i}:=G \cap W_{I_{i}}$ for all $i$. Then $G$ is a subgroup of $G^{\prime}:=\prod_{i} G_{i} \subset W_{I}$.

For all $i$ we shall define a subgroup $H_{i} \subset G_{i}$. If $I_{i}$ is spherical, put $H_{i}=1$. If $I_{i}$ is affine, choose $N>0$ such that $w^{N}=1$ for all torsion elements $w \in W$, and put $H_{i}=\left\{g^{N} \mid g \in G_{i}\right\}$.

Finally, suppose that $I_{i}$ is non-spherical and non-affine. Then $G_{i}$ is an infinite finitely generated abelian group, not containing a subgroup isomorphic to $\mathbb{Z}^{2}$ by 6.8.2. So $G_{i}$ contains a finite index subgroup $H_{i}$ isomorphic to $\mathbb{Z}$; choose any such $H_{i}$.

Now $H:=\prod_{i} H_{i}$ is a standard free abelian subgroup and $\left[G^{\prime}: G^{\prime} \cap H\right]<\infty$, so that $[G: G \cap H]<\infty$.

A consequence of 6.8 .3 is the equivalence (b) $\Longleftrightarrow$ (c) of 4.3.1, where Coxeter groups not containing subgroups isomorphic to $\mathbb{Z}^{2}$ are classified as part of the classification of word hyperbolic Coxeter groups. Our corollary says a little more, namely, it also characterizes the subgroups isomorphic to $\mathbb{Z}^{2}$, which is not clear from Moussong's work. Of course, our theory does not tell which Coxeter groups are word hyperbolic. A question is: Can our theory be extended so as to classify word hyperbolic Coxeter groups?

Theorem 6.8.3 was subsequently used in [3], [2], [17], [14], [13], [18], [16].

\section{Appendix A. Euclidean complexes of non-positive curvature}

In this appendix we summarize what we need to know about $\mathrm{CAT}(0)$ to be able to read Appendix B. Our main reference is [9].

A.1. Metric spaces. An interval is a non-empty set $I \subset \mathbb{R}$ with $u, v \in I, u<v$ $\Longrightarrow[u, v] \subset I$. Examples of intervals are $(0,1),(0,1],(0, \infty)$.

Let $(X, d)$ be a metric space. A path in $X$ is a continuous map from an interval to $X$. The length $\lambda(\alpha)$ of a path $\alpha: I \rightarrow X$ is

$$
\sup \left\{\sum_{i} d\left(\alpha t_{i}, \alpha t_{i+1}\right) \mid n \geq 0, t_{0}, \ldots, t_{n} \in I, t_{i}<t_{i+1} \text { for all } i\right\} .
$$

A path is said to be normalized if it is of the form $\alpha:[0,1] \rightarrow X$ and of constant speed $c$, that is, $d(\alpha x, \alpha y)=c d(x, y)$ for all $x, y$.

A geodesic in $X$ is a path $\alpha: I \rightarrow X$ such that $d(\alpha x, \alpha z)=d(\alpha x, \alpha y)+$ $d(\alpha y, \alpha z)$ whenever $x<y<z$. A geodesic space is a metric space in which any two points are connected by a geodesic. A local geodesic is a path $\alpha: I \rightarrow X$ such that $I$ is a union of open subsets on which $\alpha$ is a geodesic. 
A.2. Metric complexes. We denote Euclidean $n$-space by $\mathbb{E}^{n}$ and its metric by $|x-y|$ or $d(x, y)$. Denote the $n$-sphere $\left\{x \in \mathbb{E}^{n+1}|| x \mid=1\right\}$ by $\mathbb{S}^{n}$ and its metric by $d(x, y)$.

A spherical or Euclidean cell is a compact subset of $\mathbb{S}^{n}$ or $\mathbb{E}^{n}$, respectively, which is the intersection of finitely many closed half-spaces. By metric cells we mean spherical or Euclidean cells, and similarly for metric complexes, which are to be defined later. The interior of a metric cell $X$ (with respect to the smallest linear variety containing $X$ ) is denoted $X^{0}$. A face of a metric cell $X$ is either $X$ itself or $X \cap H$ where $H$ is a hyperplane disjoint with $X^{0}$.

We collect some properties of metric cells, the proofs of which are left to the reader. Some properties are proved in [11], $\S 7$. Let $X$ be a metric cell. Then faces of $X$ are again metric cells. Intersections of faces of $X$ are again faces of $X$. The relation 'being a face of' is transitive. Every metric cell is the disjoint union of the interiors of its faces. A metric cell has only finitely many vertices (= faces with exactly one element). The convex hull of a finite subset of $\mathbb{S}^{n}$ or $\mathbb{E}^{n}$ is a metric cell. All Euclidean cells are of this form, but not so for spherical ones. Spherical cells need not even be connected, as is shown by the spherical cell consisting of two antipodal points in $\mathbb{S}^{n}$.

A.2.1 Definition. A Euclidean complex is a pair $(K, C)$ of a set $K$ and a collection $C$ of metric spaces $\left(X, d_{X}\right)$, where $X \subset K(X$ is called a cell $)$, such that

MC(1) The cells cover $K$.

$\operatorname{MC}(2)$ Every metric space $\left(X, d_{X}\right) \in C$ is isometric to a Euclidean cell.

MC(3) If $Y$ is a face of a cell $X$, then $Y$ is again a cell, and $d_{Y}=\left.d_{X}\right|_{Y \times Y}$.

MC(4) The intersection of any two cells is a face of either cell.

A spherical complex is defined as above, by replacing 'Euclidean' in $\mathrm{MC}(2)$ by 'spherical'.

For the rest of this section let $(K, C)$ denote a metric complex (that is, a spherical or Euclidean complex).

Let $S$ be a subset of a cell of $K$. By MC(3) and MC(4), there is a smallest cell containing $S$, called the support of $S$. It is denoted $\bar{S}$, and we shorten $\overline{\{x\}}$ to $\bar{x}$. For a point $x \in K$, define the star $\operatorname{St}(x)$ to be the union of all cells containing $x$, and the open star $\operatorname{Ost}(S)$ to be the union of the interiors of all cells containing $S$.

A linear path in a metric cell $X$ is a map $\alpha$ from an interval $I$ to $X$ of the following form.

- If $X \in \mathbb{E}^{n}$ is Euclidean: $\alpha(t)=x+t y, x, y \in \mathbb{E}^{n}$.

- If $X \in \mathbb{S}^{n}$ is spherical: $\alpha(t)=(\cos \omega t) u+(\sin \omega t) v, u, v \in \mathbb{S}^{n} \subset \mathbb{E}^{n+1}$, $u \perp v, \omega \in \mathbb{R}_{\geq 0}$, (that is, along a 'big circle').

The numbers $|y|$ and $\omega$ are called the speed of $\alpha$. 
A linear path in $K$ is a map $\alpha$ from an interval $I$ to $K$ such that $\alpha(I)$ is contained in a cell $X$, and $\alpha$ is linear with respect to $X$. A piecewise linear path is a map $\alpha$ from an interval $I$ to $K$ with the property that for all $t \in I$ there exists $\varepsilon>0$ such that $\alpha$ is linear on $I \cap[t-\varepsilon, t]$ and $I \cap[t, t+\varepsilon]$. Since we will consider only piecewise linear paths, we call them simply paths. The speed $\operatorname{speed}(\alpha, t)$ is defined to be the speed of a linear restriction of $\alpha$ to an interval containing $t$. Thus, speed $(\alpha, t)$ is defined outside a discrete set of values of $t$. The length $\lambda(\alpha)$ is defined in Section A.1 and it is also $\int_{t \in I} \operatorname{speed}(\alpha, t) d t$.

Recall that a path $\alpha: I \rightarrow K$ is called normalized if $I=[0,1]$ and $\alpha$ has constant speed. Note that a path on a compact interval has the form $\alpha:\left[a_{0}, a_{n}\right] \rightarrow K$, where for some $a_{0}<a_{1}<\cdots<a_{n}$, the restriction $\alpha \mid\left[a_{i}, a_{i+1}\right]$ is linear for each $i$. If, in addition, $\alpha$ is normalized, then $\alpha$ is uniquely determined by the symbol $\left[\alpha\left(a_{0}\right), \ldots, \alpha\left(a_{n}\right)\right]$, which may therefore serve as a notation for $\alpha$. If $\alpha:[a, b] \rightarrow K$ is a path, we say it is a path from $\alpha(a)$ to $\alpha(b)$.

Define a pseudometric on $K$, denoted $d$, as follows. For $x, y \in K, d(x, y)$ is the infimum of lengths of paths from $x$ to $y$. If there is no such path, then $d(x, y)=\infty$. If $x, y$ are in one cell $X$, we have $d(x, y) \leq d_{X}(x, y)$, but equality does not always hold, that is, there may be a shortcut through other cells. We say that $y$ is between $x$ and $z$ if $d(x, z)=d(x, y)+d(y, z)$. So if $K$ is geodesic, then $y$ is between $x$ and $z$ if and only if there exists a geodesic from $x$ to $z$ passing through $y$.

By viewing $K$ as the quotient of the disjoint union of cells modulo identifications, we find a topology on $K$. Concretely, $U \subset K$ is open if and only if for all cells $X$, the intersection $U \cap X$ is open in $X$ (in the usual sense).

Let Shapes $(K)$ denote the set of isometry classes of cells of $K$.

A.2.2 Theorem. Suppose that Shapes $(K)$ is finite and $K$ is connected. Then $K$ is a complete geodesic space.

Proof. See [9], Theorem 7.19.

A.3. Curvature. Let $X \subset M^{n}=\mathbb{E}^{n}$ or $\mathbb{S}^{n}$ be a metric cell, $x \in X$, and let $T_{x}\left(M^{n}\right)$ denote the tangent space at $x$ to $M^{n}$. We recall that on $T_{x}\left(M^{n}\right)$ there exists a natural positive definite quadratic form, which we denote by $|y|^{2}$, and we have an exponential map exp: $T_{x}\left(M^{n}\right) \rightarrow \mathbb{E}^{2}$ defined by: $f: t \mapsto \exp (t y)$ is a constant speed geodesic and $d f / d t(0)=y$. The link $\operatorname{Lk}(x, X)$ and complement $\operatorname{Cp}(x, X)$ are defined by

$$
\begin{aligned}
& \operatorname{Lk}(x, X)=\left\{y \in T_{x}\left(M^{n}\right)|| y \mid=1 ; \text { there exists } \varepsilon>0 \text { so that } \exp ([0, \varepsilon] y) \subset X\right\}, \\
& \operatorname{Cp}(x, X)=\left\{y \in \operatorname{Lk}(x, X) \mid y \perp T_{x}(\bar{x}) \subset T_{x}\left(M^{n}\right)\right\} .
\end{aligned}
$$

These are spherical cells. If $X$ is a face of $Y$ and $x \in X$, then $\operatorname{Lk}(x, X)$ is a face of $\operatorname{Lk}(x, Y)$. For a metric complex $K$ and $x \in K$, the link $\operatorname{Lk}(x, K)$ (or briefly $\operatorname{Lk}(x)$ ) is defined as the quotient of the disjoint union of links $\operatorname{Lk}(x, X), X$ a cell containing 
$x$, modulo identifications due to inclusions of links induced by inclusions of cells. Similarly for complements. Since links and complements depend up to isometry only on the support $\bar{x}$ of the point $x$, we write $\operatorname{Lk}(\bar{x})$ for $\operatorname{Lk}(x)$ and $\mathrm{Cp}(\bar{x})$ for $\operatorname{Cp}(x)$. The complement $\operatorname{Cp}(X, K)$ is also defined for the empty cell $X$, namely, $\operatorname{Cp}(\varnothing, K)=K$.

For all $x \in K$, we have a natural projection $\operatorname{St}(x)-\{x\} \rightarrow \operatorname{Lk}(x)$.

Let $S(\lambda)$ denote the circle (viewed as a one-dimensional Euclidean or spherical complex) of length $\lambda$. A cycle in a metric complex $K$ is a piecewise linear map $\alpha: S(\lambda) \rightarrow K$. A minimal cycle is a non-constant cycle which is a local geodesic.

Roughly, a CAT $(\kappa)$ space (for $\kappa \in \mathbb{R}$ ) is a geodesic space whose triangles are no fatter than those in the spaces of constant curvature $\kappa$. For the full definition, which we shall not need, we refer to [9]. For $\kappa=0$ this means that we are comparing with Euclidean space $\mathbb{E}^{n}$ and for $\kappa=1$ with the metric spheres $\mathbb{S}^{n}$.

The main aim of this appendix is to recognise from local data whether a Euclidean complex is $\operatorname{CAT}(0)$, a global condition.

A.3.1 Definition. A metric complex is said to satisfy the link condition if for every vertex $v \in K$, the link $\operatorname{Lk}(v, K)$ is $\operatorname{CAT}(1)$. A spherical complex is said to satisfy the girth condition if every minimal cycle has length at least $2 \pi$.

A.3.2 Theorem. (a) Let $K$ be a Euclidean complex with finitely many shapes. Then $K$ is $\mathrm{CAT}(0)$ if and only if it is simply connected and satisfies the link condition.

(b) Let $K$ be a spherical complex with finitely many shapes. Then $K$ is CAT(1) if and only if it satisfies the link condition and the girth condition.

Proof. See [9], Theorem 5.4.

A.3.3 Example. We give an example of a spherical complex which satisfies the girth condition but is not CAT(1). It is $K=S(\lambda) *$ \{point where $0<\lambda<2 \pi$. Here $*$ denotes the spherical join; see [9], Definition 5.13, for a definition. Then $K$ contains no minimal cycles, so it satisfies the girth condition. However, its link at the pole is a circle of length $\lambda<2 \pi$, so $K$ is not CAT(1).

We now explain the transitivity of complements. Let $K$ be a metric complex and let $X \subset Y \subset K$ be cells. Then $\operatorname{Cp}(X, Y)$ can be embedded naturally as a cell in $\mathrm{Cp}(X, K)$, and

$$
\mathrm{Cp}(Y, K) \cong \mathrm{Cp}(\mathrm{Cp}(X, Y), \mathrm{Cp}(X, K)) .
$$

Conversely, every cell of $\mathrm{Cp}(X, K)$ has the form $\mathrm{Cp}(X, Y)$.

A.4. Convex metrics. A function $f: \mathbb{R} \rightarrow \mathbb{R}$ is said to be convex if for all $x, y \in I$ and all $t \in[0,1]$ we have

$$
f((1-t) x+t y) \leq(1-t) f(x)+t f(y) .
$$


A geodesic space is said to have convex metric if for any two normalized geodesics $\alpha, \beta$ the function $f: t \mapsto d(\alpha(t), \beta(t))$ is convex.

A.4.1 Proposition. Every CAT(0) space has convex metric.

Proof. See [9], Proposition 2.2.

\section{Appendix B. The Davis-Moussong complex}

In the first three sections of this appendix we rewrite Moussong's thesis, where a Euclidean complex $M$ of non-positive curvature is constructed, on which a Coxeter groups $W$ acts. In Section B.4 we give an alternative construction, which is shorter and can be read independently. In Section B.5, the metric of $M$ is compared to the distance in $W$. In Section B.6 we derive an exponential solution to the conjugacy problem.

B.1. Nerves of almost negative matrices. An almost negative matrix is a square symmetric real matrix $A=\left(a_{i j}\right)$ with $a_{i j} \leq 0$ if $i \neq j$. Given an almost negative matrix $A=\left(a_{i j}\right)$, we define a spherical complex $N=N(A)$, called the nerve of $A$, as follows. We like to think of the rows and columns of $A$ as indexed by some set $I$. Let $E$ be a real vector space with basis $\left\{e_{i} \mid i \in I\right\}$. Define a symmetric bilinear form $(\cdot, \cdot)$ on $E$ by $\left(e_{i}, e_{j}\right)=a_{i j}$. For a subset $J \subset I$, let $E_{J}$ denote the subspace of $E$ generated by $\left\{e_{i} \mid i \in J\right\}$. We call a subset $J \subset I$ spherical if the bilinear form restricted to $E_{J}$ is positive definite. For spherical $J \subset I$, define a spherical cell $S_{J}$ (which is in fact a simplex, that is, the dimension is the number of vertices minus one) by

$$
S_{J}=\left\{x=\sum_{i \in J} x_{i} e_{i}\left|x_{i} \geq 0,\right| x \mid=\sqrt{(x, x)}=1\right\},
$$

which inherits the structure of a spherical cell by its embedding in the Euclidean space $E_{J}$. Now $N(A)$ is defined to be the spherical complex with the union of the $S_{J}$ as underlying point set and the $S_{J}$ as cells (noting $S_{\varnothing}=\varnothing$ ).

We call an almost negative matrix normalized if all diagonal elements are equal to 1 . To every almost negative matrix $A=\left(a_{i j}\right)_{i, j \in I}$ we associate a normalized matrix norm $(A)=B$ as follows. The rows and columns of $B$ are indexed by $J=$ $\left\{i \in I \mid a_{i i}>0\right\}$ and $b_{i j}=a_{i j} / \sqrt{a_{i i} a_{j j}}$. The nerves of $A$ and $\operatorname{norm}(A)$ are isomorphic.

B.1.1 Theorem. Nerves of almost negative matrices are CAT(1).

Proof. See [25]. 
B.1.2 Lemma. Let $N$ be the nerve of an almost negative matrix, and let $x \in N$. Then $\mathrm{Cp}(x, N)$ is isometric to the nerve of some almost negative matrix.

Proof. Retain the denotations of $I, A, E, e_{i}, E_{J}$, so that $N=N(A)$. We may assume $A$ to be normalized. By transitivity of complements, we may suppose $x$ to be a vertex of $N$, say $x=e_{i}$. Let $f_{j}$ be the orthogonal projection of $e_{j}$ on $e_{i}^{\perp}$, the orthogonal set in $E$ to $e_{i}$. Thus, for pairwise different indices $i, j, k$ we have

$$
f_{j}=e_{j}-\left(e_{j}, e_{i}\right) e_{i}=e_{j}-a_{i j} e_{i}
$$

and

$$
\begin{aligned}
\left(f_{j}, f_{k}\right) & =\left(e_{j}-a_{i j} e_{i}, e_{k}-a_{i k} e_{i}\right) \\
& =a_{j k}-2 a_{i j} a_{i k}+a_{i j} a_{i k} a_{i i}=a_{j k}-a_{i j} a_{i k} \leq 0
\end{aligned}
$$

since $a_{j k}, a_{i j}, a_{i k} \leq 0$. Hence the matrix $B=\left(f_{j}, f_{k}\right)_{j, k \neq i}$ is again an almost negative matrix. We claim that $\mathrm{Cp}\left(e_{i}, N\right) \cong N(B)$. Let $E_{J}$ be positive definite with $i \in J$, and let $A^{\prime}, B^{\prime}$ be the restrictions of $A, B$ to $J, J-\{i\}$, respectively. Let $S=\left\{x \in E_{J}|| x \mid=1\right\}$. Consider the natural map from the tangent space $T_{e_{i}}(S)$ to $e_{i}^{\perp}$ (which can be defined as translation over $-e_{i}$, viewing $T_{e_{i}}(S)$ as a linear variety in $\left.E_{J}\right)$. This map restricts to an isometry $\mathrm{Cp}\left(e_{i}, N\left(A^{\prime}\right)\right) \rightarrow N\left(B^{\prime}\right) \subset e_{i}^{\perp}$. Using the fact that for all $J \subset I-\{i\}$, the subspace $\operatorname{Span}\left\{f_{j} \mid j \in J\right\}$ is positive definite if and only if $E_{J \cup\{i\}}$ is, it follows that $\mathrm{Cp}\left(e_{i}, N\right) \cong N(B)$.

B.2. Cells for the Davis-Moussong complex. For the rest of this appendix, we consider a Coxeter system $(W, S)$ and retain the notations of Section 1. In this section fix positive real numbers $a_{s}(s \in S)$.

Let $J \subset S$ be spherical. Since $E_{J}=\operatorname{Span}\left\{e_{s} \mid s \in J\right\}$ is non-degenerate, there exists a unique basis $\left\{f_{s}^{J} \mid s \in J\right\}$ of $E_{J}$ dual to $\left\{e_{s}\right\}$, defined by $\left(f_{s}^{J}, e_{t}\right)=\delta_{s t}$ $(s, t \in J)$. Writing $C h$ for the convex hull, we define

$$
\begin{aligned}
x_{J} & =\sum_{s \in J} a_{s} f_{s}^{J}, \quad X_{J}=\mathrm{Ch}\left(W_{J} x_{J}\right), \\
X_{J K} & =\operatorname{Ch}\left(W_{J} x_{K}\right) \text { for } J, K \subset S \text { both spherical with } J \subset K .
\end{aligned}
$$

The sets $X_{K}$ and $X_{J K}$ are Euclidean cells by their embedding in the Euclidean space $E_{K}$. For spherical $J \subset S$, let $p_{J}: E \rightarrow E_{J}$ denote the orthogonal projection. It is well defined since the quadratic form on $E_{J}$ is non-degenerate.

B.2.1 Definition. For spherical $J, K \subset S$ such that $J \subset K$, we define $g_{J}^{K}=$ $\sum_{s \in K-J} f_{s}^{K}$.

B.2.2 Lemma. (a) $\operatorname{dim} X_{J}=\# J$.

(b) Let $J \subset K$ be spherical subsets of $S$. Then $p_{J} x_{K}=x_{J}$. Moreover, it follows that $p_{J} \mid X_{J K}: X_{J K} \rightarrow X_{J}$ is an isometry of cells, equivariant under $W_{J}$. 
(c) The non-empty faces of $X_{K}$ are precisely all $w X_{J K}$ for all $J \subset K, w \in W_{K}$. For all $J \subset K, x \in X_{K}$ we have $\left(g_{J}^{K}, x-x_{K}\right) \leq 0$, with equality if and only if $x \in X_{J K}$.

Proof. (a) For all $s \in J$ we have $s x_{J}=x_{J}-2\left(x_{J}, e_{s}\right) e_{s}$. Since $\left(x_{J}, e_{s}\right)=a_{s}>0$ and since the $e_{s}(s \in J)$ are linearly independent, the convex hull of $\left\{x_{J}\right\} \cup\left\{s x_{J} \mid\right.$ $s \in J\}$ has dimension \# $J$. Hence so has $X_{J}$.

(b) $E$ is the orthogonal direct sum of $E_{J}$ and $E_{J}^{\perp}$, and $W_{J}$ fixes $E_{J}^{\perp}$ pointwise and stabilizes $E_{J}$. It follows that $p_{J}$ is $W_{J}$-equivariant and $p_{J}$ sets up an isometry from $X_{J K}$ to its image. By definition of orthogonal projection, for $s \in J$, we have $\left(p_{J} x_{K}, e_{s}\right)=\left(x_{K}, e_{s}\right)=a_{s}$. It follows that $p_{J} x_{K}=x_{J}$ and $p_{J} X_{J K}=X_{J}$.

(c) From B.2.3, choosing $x=x_{K}, p=g_{J}^{K}, W=W_{K}$, we find that

$$
\left(g_{J}^{K}, w x_{K}-x_{K}\right) \leq 0,
$$

with equality only for $w$ fixing $g_{J}^{K}$, that is, $w \in W_{J}$ by 1.2.2 (c). The latter statement of (c) follows. Thus, $X_{J K}$ is a face of $X_{K}$. Conversely, each face of $X_{K}$ containing $x_{K}$ is of the form $X=\left\{x \in X_{K} \mid\left(p, x-x_{K}\right)=0\right\}$, where $p \in E_{K}$ and $\left(p, x-x_{K}\right) \leq 0$ for all $x \in X_{K}$. The latter condition implies, by choosing $x=s x_{K}, s \in K$, that we can write $p=\sum_{s \in J} c_{s} f_{s}^{K}, c_{s}>0$ for some subset $J \subset K$. By an argument similar to the above, the vertex set of $X$ is $W_{J} x_{K}$, whence $X=X_{J K}$. Note that $x_{K}$ is a vertex of $X_{K}$ because the vertex set of $X_{K}$ is a non-empty subset of $W_{K} x_{K}$ and $W_{K}$-invariant. Hence every non-empty face of $X_{K}$ is of the form $w X_{J K}, w \in W_{K}$, $J \subset K$.

B.2.3 Lemma. Let $W$ be a finite Coxeter group, and identify $E$ with its dual $E^{*}$ by $(\cdot, \cdot)=\langle\cdot, \cdot\rangle$. Let $p \in \bar{C}, x \in C, w \in W$. Then $(p, w x) \leq(p, x)$, with equality only if $w p=p$.

Proof. Induction on $\ell(w)$. If $w=1$ it is clear. If $w=s v, s \in S, \ell(v)<\ell(w)$, then $\left(v x, e_{s}\right)>0$ by 1.2.2(b). Hence by induction $(p, w x)=\left(p, v x-2\left(v x, e_{s}\right) e_{s}\right)=$ $(p, v x)-2\left(v x, e_{s}\right)\left(p, e_{s}\right) \leq(p, v x) \leq(p, x)$. Now suppose that equality holds. Then $\left(p, e_{s}\right)=0$ and $(p, v x)=(p, x)$. Hence $s p=p$ and, by induction, $v p=p$. We conclude that $w p=p$.

B.3. The Davis-Moussong complex. In this section we will construct a Euclidean complex introduced by Moussong [43], 14.1, and we will prove its main properties B.3.2 and B.3.3. We call it the Davis-Moussong complex and denote it by $M(W)$ or $M$. A difference between Moussong's approach and ours is that Moussong builds the complex from one fundamental domain, whereas we build it from cells, that is, the approaches are dual. 
By a pre-cell we mean a subset of $W$ of the form $w W_{J}, w \in W, J \subset S$ spherical. For each pre-cell $p$, fix an element $w_{p} \in p$. Then $p=w_{p} W_{J(p)}$ for a unique spherical $J(p) \subset S$.

B.3.1 Lemma. The intersection of any two pre-cells is either again a pre-cell, or the empty set. Moreover, if $p, q$ are pre-cells with non-empty intersection, then $J(p) \cap$ $J(q)=J(p \cap q)$.

Proof. This follows from 1.2.3(c).

Let $\widehat{M}$ denote the set of pairs $(p, x)$ where $p$ is a pre-cell and $x \in X_{J(p)}$. Define an equivalence relation on $\hat{M}$ by

$$
(p, x) \sim(q, y) \Longleftrightarrow\left\{\begin{array}{l}
r:=p \cap q \neq \varnothing, \\
w_{r}^{-1} w_{p} x \in X_{J(r) J(p)}, w_{r}^{-1} w_{q} y \in X_{J(r) J(q)}, \\
p_{J(r)}\left(w_{r}^{-1} w_{p} x-w_{r}^{-1} w_{q} y\right)=0 .
\end{array}\right.
$$

Now the underlying set of $M$ is $\widehat{M}$ modulo this equivalence relation, and the cells are the empty set and the images in $M$ of $\left\{(p, x) \mid x \in X_{J}(p)\right\}$. Since these sets inject into $M$, they still carry structures of Euclidean cells. The metric on $M$ is denoted by $d_{M}$.

B.3.2 Proposition. $M(W)$ is a locally finite Euclidean complex. The group $W$ acts discretely on it with finitely many orbits of cells. The action of $W$ on the vertex set is simply transitive.

Proof. Straightforward, using B.2.2 and B.3.1.

Let $v$ denote the vertex of $M$ which is the equivalence class of $\left(\{1\}, x_{\varnothing}\right) \in \hat{M}$. Then the neighbours (in the 1-skeleton of $M$ ) of $v$ are $s v, s \in S$, and the corresponding edges have length $2 a_{s}$. We identify $W$ with the vertex set of $M$ by $w \mapsto w v$. Let $Y_{J}$ denote the images in $M$ of $\left\{\left(W_{J}, x\right) \mid x \in X_{J}\right\}$. Thus, the cells of $M$ are the sets $w Y_{J}, w \in W, J \subset S$ spherical.

B.3.3 Theorem (Moussong). $M(W)$ satisfies the link condition.

Proof. Let $A$ be the Gram matrix of $(W, S)$, that is, $A=\left(a_{s t}\right)_{s, t \in S}, a_{s t}=\left(e_{s}, e_{t}\right)=$ $-\cos \left(\pi / m_{s t}\right)$. Using 1.2.6, it is easy to see that for every vertex $x$ of $M, \mathrm{Cp}(x, M) \cong$ $N(A)$. From B.1.2 and transitivity of complements, it follows that for every point $y \in M$, the complement $\mathrm{Cp}(y, M)$ is the nerve of some almost negative matrix. By B.1.1, $\mathrm{Cp}(y, M)$ satisfies the girth condition. By A.3.2, $M$ satisfies the link condition. 
B.4. The embedding of the Davis-Moussong complex into the Tits cone. We will give an alternative definition of the Davis-Moussong complex, by embedding it into $U^{0}$, the interior of the Tits cone.

Fix a point $v \in C$. We define the underlying point-set of the alternative DavisMoussong complex by

$$
M^{\prime}=\bigcup\left\{\operatorname{Ch}\left(w W_{J} v\right) \mid J \subset S \text { spherical }, w \in W\right\} .
$$

The set $\operatorname{Ch}\left(W_{J} v\right)$ (and thereby $\mathrm{Ch}\left(w W_{J} v\right)$ for all $w \in W$ ) is given the structure of a Euclidean cell by the embedding $q^{-1} r: \operatorname{Ch}\left(W_{J} v\right) \rightarrow E_{J}$, where $r: E^{*} \rightarrow E_{J}^{*}$ is the restriction map and $q: E_{J} \rightarrow E_{J}^{*}$ is the isomorphism associated to the inner product $(\cdot, \cdot)$ on $E_{J}$, that is, $q x=(x, \cdot)$. Let $C$ be the collection of the cells $\operatorname{Ch}\left(w W_{J} v\right)$ where $w \in W, J \subset S$ spherical.

B.4.1 Proposition. The pair $\left(M^{\prime}, C\right)$ is a Euclidean complex and is isomorphic to the Davis-Moussong complex $M$. The edge from $v$ to sv has length $2\left\langle v, e_{s}\right\rangle$.

Proof. We will prove one step, namely that the intersection of any two cells is a face of either cell. The rest of the proof is straightforward and left to the reader.

Let $X=\mathrm{Ch}\left(g W_{I} v\right), Y=\mathrm{Ch}\left(h W_{J} v\right)$ be cells with non-empty intersection. First we will prove that $X$ and $Y$ have a vertex in common, that is, $g W_{I} \cap h W_{J} \neq \varnothing$.

Suppose that $g W_{I} \cap h W_{J}=\varnothing$, and let $x \in g W_{I}, y \in h W_{J}$ such that $d_{W}(x, y)$ is minimal. Let $\alpha$ be a root separating $x$ from $y$, say $\langle x v, \alpha\rangle>0,\langle y v, \alpha\rangle<0$. Since $g W_{I}$ is gated by $1.3 .5(\mathrm{c})$, and $d_{W}\left(g W_{I}, y\right)=d_{W}(x, y)$, we have $\left\langle x^{\prime} v, \alpha\right\rangle>0$ for all $x^{\prime} \in g W_{I}$. Hence $\langle X, \alpha\rangle \subset \mathbb{R}_{>0}$. Similarly $\langle Y, \alpha\rangle \subset \mathbb{R}_{<0}$, which shows $X \cap Y=\varnothing$, a contradiction. Hence $g W_{I} \cap h W_{J} \neq \varnothing$.

After left multiplication by some element of $W$, we may suppose that $1 \in g W_{I} \cap$ $h W_{J}$ and so $X=\mathrm{Ch}\left(W_{I} v\right), Y=\mathrm{Ch}\left(W_{J} v\right)$.

Write $K=I \cap J$. We will show that $X \cap Y=\mathrm{Ch}\left(W_{K} v\right)$. Let $y \in E^{*}$ such that $v+y \in X \cap Y$. We claim that $y \in \operatorname{Span}\{s v-v \mid s \in K\}$.

Let us first show how the claim proves the promised result. We have

$$
v+y \in(v+\operatorname{Span}\{s v-v \mid s \in K\}) \cap \operatorname{Ch}\left(W_{I} v\right)=\operatorname{Ch}\left(W_{K} v\right),
$$

where the latter equality follows since $\mathrm{Ch}\left(W_{K} v\right)$ is a face of $\mathrm{Ch}\left(W_{I} v\right)$ by B.2.2.

We can write

$$
y=\sum_{s \in I} a_{s}(s v-v)=\sum_{s \in K} b_{s}(s v-v), \quad a_{s}, b_{s} \geq 0 .
$$

In order to prove the claim, we may suppose that $K=\varnothing$; for otherwise, for each $s \in K$, subtract $\min \left(a_{s}, b_{s}\right)$ from $a_{s}$ and $b_{s}$, and then replace $I$ and $J$ by $\{s \in I \mid$ 
$\left.a_{s} \neq 0\right\}$ and $\left\{s \in J \mid b_{s} \neq 0\right\}$, respectively. Let $t \in J$. We have

$$
\begin{aligned}
\left\langle y, e_{t}\right\rangle & =\sum_{s \in I} a_{s}\left\langle s v-v, e_{t}\right\rangle=\sum_{s \in I} a_{s}\left\langle v, s e_{t}-e_{t}\right\rangle \\
& =\sum_{s \in I} a_{s}\left\langle v,-2 a_{s t} e_{s}\right\rangle=\sum_{s \in I}-2 a_{s} a_{s t}\left\langle v, e_{s}\right\rangle \geq 0,
\end{aligned}
$$

since every term in the last sum is non-negative. In the same way it follows that

$$
\left\langle y, e_{t}\right\rangle=\sum_{s \in J}-2 b_{s} a_{s t}\left\langle v, e_{s}\right\rangle .
$$

In matrix form this reads $A_{J} b=c$, where $A_{J}$ is the matrix $\left(a_{s t}\right)_{s, t \in J}$, and $b$ and $c$ are the vectors $b=\left(-2 b_{s}\left\langle v, e_{s}\right\rangle\right)_{s \in J}, c=\left(\left\langle y, e_{t}\right\rangle\right)_{t \in J}$. By (23), $c$ is totally non-negative. By 1.5.2, so is $A_{J}^{-1}$. Hence so is $b=A_{J}^{-1} c$. Since $\left\langle v, e_{s}\right\rangle>0$ for all $s \in S$, it follows that $b_{s}=0$ for all $s \in J$. Hence $y=0$, which proves the claim.

B.5. Comparison of $\boldsymbol{d}_{\boldsymbol{W}}$ and $\boldsymbol{d}_{\boldsymbol{M}}$. Let $M$ be the Davis-Moussong complex of a Coxeter system $(W, S)$. Let $\Gamma$ denote the 1-skeleton of $M$. The object of this section is to compare the path metric of $\Gamma$, denoted $d_{\Gamma}$, with the restriction of $d_{M}$ to $\Gamma$. Clearly, we have $d_{M} \leq d_{\Gamma}$. Milnor proved (in a more general setting) that there exist $K, L \in \mathbb{R}$ such that $d_{\Gamma} \leq K d_{M}+L$. His proof is non-effective, that is, it does not give us $K$ and $L$ explicitly. We will give explicit, though quite weak bounds for $K$ and $L$ in B.5.6.

Let us identify $W$ with the vertex set in $M$, such that the left actions of $W$ coincide. We write $\ell_{\Gamma}(x)=d_{\Gamma}(1, x)(x \in \Gamma)$ and $\ell_{M}(x)=d_{M}(1, x)(x \in M)$.

B.5.1 Lemma. Let $N \subset M$ be a set containing 1 such that $M$ is covered by $\{w N \mid$ $w \in W\}$. Let $F=\{f \in W \mid N \cap f N \neq \varnothing\}$. Let there be given $\delta, R>0$ such that

$$
\begin{array}{rlrl}
N \cap w N & =\varnothing \Longrightarrow d(N, w N) \geq \delta & & \text { for all } w \in W, \\
\ell_{\Gamma}(f) & \leq R & \text { for all } f \in F .
\end{array}
$$

Then $\ell_{\Gamma}(x) \leq\left(\ell_{M}(x) / \delta+1\right) R$ for all $x \in W$.

Proof. Let $x \in W, \lambda=\ell_{M}(x)$. Let $1=x_{0}, x_{1}, \ldots, x_{t}=x$ be a sequence of points in $M$ such that $d_{M}\left(x_{i}, x_{i+1}\right)<\delta, t \leq \lambda / \delta+1$. Let $h_{i} \in W$ such that $x_{i} \in h_{i} N, h_{0}=1, h_{t}=x$. Write $f_{i}=h_{i-1}^{-1} h_{i}$, so that $x=f_{1} f_{2} \ldots f_{t}$. Since $d_{M}\left(x_{i}, x_{i+1}\right)<\delta$, we have $h_{i} N \cap h_{i+1} N \neq \varnothing$, whence $f_{i} \in F$. Hence $\ell_{\Gamma}(x) \leq \sum \ell_{\Gamma}\left(f_{i}\right) \leq t R \leq(\lambda / \delta+1) R$.

The following proposition was proved by Milnor [42], Lemma 2. 
B.5.2 Proposition. There exist $K, L \in \mathbb{R}$ such that $\ell_{\Gamma}(x) \leq K \ell_{M}(x)+L$ for all $x \in W$.

Proof. We know that a subset of $M$ is compact if and only if it is bounded and closed, and by B.3.2 that $W$ acts discretely on $M$ with compact quotient. It follows easily that the conditions of B.5.1 are fulfilled by certain $N, \delta, R$, whence the proposition. As an example, we prove the existence of $\delta$ satisfying (24). Suppose that $N$ is compact. Let $w_{1}, w_{2}, \ldots \in W$, let $d\left(N, w_{i} N\right) \rightarrow 0(i \rightarrow \infty)$, and let $N \cap w_{i} N=\varnothing$. Since $N$ is bounded, $\ell_{M}\left(w_{i}\right)$ is bounded. Since $W$ is discrete in $M$, the $w_{i}$ have a stable infinite subsequence. Hence for $w_{i}$ in this subsequence, $d\left(N, w_{i} N\right)=0$, which implies that $N \cap w_{i} N \neq \varnothing$, a contradiction.

The above proof is not effective. More precisely, the proof of the existence of $\delta$ is non-constructive. We proceed to give an effective, though quite weak bound for $K$ and $L$.

B.5.3 Lemma. Let $K$ be a Euclidean complex. Let there be given $\delta>0$ such that for every cell $X$ of $K$ and all $x, y \in X$, we have

$$
d_{X}(x, y)=d_{X}(\bar{x}, y)<\delta \Longrightarrow x \in \bar{y} .
$$

Then any two disjoint cells in $K$ have distance at least $\delta$.

Proof. Let $X, Y$ be cells with distance smaller than $\delta$. Let $\alpha$ be a normalized geodesic from say $x \in X$ to $y \in Y$ of length $\lambda(\alpha)=d(X, Y)<\delta$. Let $0=t_{0}<t_{1}<\cdots<$ $t_{n}=1$ be such that $\alpha \mid\left[t_{i}, t_{i+1}\right]$ is linear, and write $x_{i}=\alpha\left(t_{i}\right)$. By induction on $i$ we will prove

$$
\alpha\left(\left[0, t_{i}\right]\right) \subset \overline{x_{i}} .
$$

For $i=0$ this is clear. Let $i>0$. Let $Z$ be a cell containing $\alpha\left(\left[t_{i-1}, t_{i}\right]\right)$. We have $x_{i-1}=\alpha\left(t_{i-1}\right) \in \alpha\left(\left[t_{i-1}, t_{i}\right]\right) \subset Z$, whence $\overline{x_{i-1}} \subset Z$. Hence, by induction, $\alpha\left(\left[0, t_{i}\right]\right) \subset Z$. Since $\alpha$ is a constant speed geodesic, $\alpha \mid\left[0, t_{i}\right]$ is linear. From $\lambda(\alpha)=$ $d(X, Y)$ it follows that $\lambda\left(\alpha,\left[0, t_{i}\right]\right)=d_{Z}\left(\bar{x}, x_{i}\right)$. Since also $\lambda(\alpha)<\delta$, we may conclude that $x \in \overline{x_{i}}$. It easily follows that $\alpha\left(\left[0, t_{i}\right]\right) \subset \overline{x_{i}}$, which proves (26). Choosing $i=n$ in (26), we find $\operatorname{Im} \alpha \subset \bar{y}$. In particular, $x=\alpha(0) \in \bar{y}$. Hence $X \cap Y \neq \varnothing$, which finishes the proof.

Note that many complexes do not satisfy the condition of B.5.3 (for all $\delta>0$ ). For example, suppose that some cell $X$ is a Euclidean triangle with vertices $a, b, c \in \mathbb{E}^{2}$, having a sharp angle at $a$. Choose $y \in[a, b]$ close to $a$, and let $x$ be the projection of $y$ on $[a, c]$. Then, if $y$ is close enough to $a, d_{X}(x, y)=d_{X}(\bar{x}, y)<\delta$ but $x \notin \bar{y}$. The next two lemmas show that the Davis-Moussong complex is special in the sense that B.5.3 can be applied to it. 
Lemma B.5.4 is an effective version of what can be done for every Euclidean complex with only finitely many isometry classes of cells. Lemma B.5.5 shows a special property of the Davis-Moussong complex, which connects Lemmas B.5.3 and B.5.4.

\section{B.5.4 Lemma. Let}

$$
\delta=\min \left\{\frac{2 a_{s}}{\left|g_{J}^{K}\right|} \mid J, K \subset S \text { spherical, } J \subset K, s \in K-J\right\} .
$$

Then for every cell $X \subset M$ and all $x, y \in X$, we have

$$
d(x, y)<\delta \Longrightarrow \bar{x} \cap \bar{y} \neq \varnothing .
$$

Proof. Identify $X$ with $X_{K}$. We may suppose that $x_{K} \in \bar{x}$, say $\bar{x}=X_{J K}, J \subset K$. Let us write $h_{J}^{K}=g_{J}^{K} /\left|g_{J}^{K}\right|$, so that $\left|h_{J}^{K}\right|=1$. Since

$$
\left(h_{J}^{K}, x_{K}-s x_{K}\right)=\frac{\left(g_{J}^{K}, 2 a_{s} e_{s}\right)}{\left|g_{J}^{K}\right|}=\frac{2 a_{s}}{\left|g_{J}^{K}\right|},
$$

for $s \in K-J$, it follows from the definition of $\delta$ that

$$
\left(h_{J}^{K}, x_{K}-z\right) \geq \delta
$$

for every vertex $z$ adjacent to $x_{K}$, not in $X_{J K}$. By $W_{J}$-invariance of $h_{J}^{K}$, (27) holds for every vertex $z$ adjacent to a vertex of $X_{J K}$ but which is not in $X_{J K}$ itself. Using the fact that $\left(h_{J}^{K}, x_{K}-z\right)=0$ for all $z \in X_{J K}$, it easily follows that (27) holds for every vertex $z$ of $X_{K}$ which is not in $X_{J K}$.

Now suppose that $d(\bar{x}, y)<\delta$. Then $\left(h_{J}^{K}, x_{K}-y\right)<\delta$, since $\left|h_{J}^{K}\right|=1$. Hence $\left(h_{J}^{K}, x_{K}-z\right)<\delta$ for some vertex $z$ of $\bar{y}$. Hence $z \in \bar{x} \cap \bar{y}$.

B.5.5 Lemma. Let $K \subset S$ be spherical, and let $x, y \in X_{K}=Z$. Then

$$
\left[d_{Z}(x, y)=d_{Z}(\bar{x}, y) \text { and } \bar{x} \cap \bar{y} \neq \varnothing\right] \Longrightarrow x \in \bar{y} .
$$

Proof. We may suppose that $x \in \bar{x} \cap \bar{y}$. Write $\bar{x}=X_{J K}, \bar{y}=X_{L K}$. Suppose that $x \notin \bar{y}$, so that $\bar{x} \not \subset \bar{y}$, that is, $J \not \subset L$. Let $s \in J-L$.

Since $d(\bar{x}, y)=d(x, y)$, the projection of $y$ on $E_{J}+x_{K}$ (= smallest linear variety containing $X_{J K}$ ) is in the interior $X_{J K}^{0}$ of $X_{J K}$. Equivalently, $p_{J} y \in X_{J}^{0}$. In particular, by B.2.2 (c),

$$
\left(f_{s}^{J}, p_{J}\left(y-x_{K}\right)\right)<0 .
$$

For all $t \in L$ we have

$$
\left(f_{s}^{J}, p_{J}\left(t x_{K}-x_{K}\right)\right)=\left(f_{s}^{J}, p_{J}\left(-2 a_{t} e_{t}\right)\right)=-2 a_{t}\left\langle f_{s}, p_{J} e_{t}\right\rangle \geq 0,
$$

by 2.2.2. Since $y-x_{K}$ is a linear combination of $t x_{K}-x_{K}(t \in L)$ with non-negative coefficients, we find $\left(f_{S}^{J}, p_{J}\left(y-x_{K}\right)\right) \geq 0$, contradicting (28). This finishes the proof. 
B.5.6 Proposition. Let $\delta$ be as defined in B.5.4. Let $R$ be twice the maximum diameter of $\Gamma_{J}=\Gamma \cap Y_{J}$ for spherical $J \subset S$. Then $\ell_{\Gamma}(x) \leq\left(\ell_{M}(x) / \delta+1\right) R$ for all $x \in W$.

Proof. We will verify the conditions of B.5.1. Choose $N$ to be the union of all $Y_{J}$ for spherical $J \subset S$. Note that $f \in F \Longleftrightarrow$ there are spherical $J, K \subset S: Y_{J} \cap f Y_{K} \neq$ $\varnothing$. Thus, $F$ is the union of all $W_{J} W_{K}$ for spherical $J, K \subset S$. Clearly, (25) is satisfied. We will show that (24) holds. By combination of B.5.4 and B.5.5, the DavisMoussong complex satisfies the condition of B.5.3. Now suppose that $d(N, w N)<\delta$. Then $d\left(Y_{J}, w Y_{K}\right)<\delta$ for some spherical $J, K \subset S$. By B.5.3, $Y_{J} \cap w Y_{K} \neq \varnothing$, whence $N \cap w N \neq \varnothing$, which proves (24). The proof is finished by applying B.5.1.

Probably B.5.6 is very far from the best possible result. We conjecture that $K$, $L$ do not have to depend on $W$, that is, there exist $K, L$ such that for every Coxeter group $W$, on choosing all edges in $M$ to have length 1 , we have $d_{\Gamma} \leq K d_{M}+L$.

B.6. The conjugacy problem. In this section we will show how the Davis-Moussong complex implies an exponential solution to the conjugacy problem. The same argument applies to every non-positively curved group. I heard the idea from M. Shapiro. In [20], a doubly exponential solution, that is, a solution of complexity

$$
A^{B^{\ell(v)+\ell(w)}}
$$

is given, also using the Davis-Moussong complex.

B.6.1 Proposition. There exist computable constants $P, Q$ such that for any two conjugate $v, w \in W$, there exists a sequence

$$
v=w_{0}, w_{1}, \ldots, w_{n}=w,
$$

such that for all $i$, we have $w_{i+1}=s w_{i}$ s for some $s \in S$, and

$$
\ell\left(w_{i}\right) \leq P[\ell(v)+\ell(w)]+Q .
$$

Proof. Choose the Davis-Moussong complex such that all edges have length 1 , and let $\Gamma$ be its 1-skeleton. Let $N=M \cap \bar{C}$, where we embed $M \subset U^{0}$, and let $x \in N$ denote the trivial vertex. Let $K, L$ be computable constants such that $d_{\Gamma} \leq K d_{M}+L$ (see B.5.6). We will show that $P:=K, Q:=L+2 K \operatorname{diam}(N)$ verify the proposition. Here $\operatorname{diam}(N)$ denotes the diameter of $N$, which is easily shown to be finite. Let $v, w \in W$ be conjugate, say $v=g^{-1} w g$. Let $\alpha, \beta$ denote the normalized geodesics in $M$ from $x$ to $g x$ and from $w x$ to $w g x=g v x$, respectively. By convexity of the metric of $M$ (Proposition A.4.1), we have

$$
\begin{aligned}
d_{M}(\alpha(t), \beta(t)) & \leq(1-t) d_{M}(x, w x)+t d_{M}(g x, g v x) \\
& \leq(1-t) \ell(w)+t \ell(v) \leq \ell(v)+\ell(w) .
\end{aligned}
$$


Consider

$$
X:=\{h \in W \mid h N \text { meets } \operatorname{Im} \alpha\} .
$$

Note that $1, g \in X$. We will show that for all $h \in X$, we have

$$
\ell\left(h^{-1} w h\right) \leq P[\ell(v)+\ell(w)]+Q .
$$

Let $h \in X$. Let $y \in h N \cap \operatorname{Im} \alpha$, say $y=\alpha(t)$, so that $w y=\beta(t)$. Then

$$
\begin{aligned}
\ell\left(h^{-1} w h\right) & =d_{\Gamma}(h x, w h x) \\
& \leq K d_{M}(h x, w h x)+L \\
& \leq K\left[d_{M}(y, w y)+2 \operatorname{diam}(N)\right]+L \\
& \leq K[\ell(v)+\ell(w)+2 \operatorname{diam}(N)]+L,
\end{aligned}
$$

which shows (29). For every point $y \in M$, the set $\{h \in W \mid y \in h N\}$ is connected. It is easy to deduce that $X$ is connected, which concludes the proof.

The above proposition suggests the following algorithm solving the conjugacy problem. Let $G$ be the graph with vertex set

$$
\{g \in C(w) \mid \ell(g) \leq P[\ell(v)+\ell(w)]+Q\}
$$

where $g, h$ are adjacent if and only if $s g s=h$ for some $s \in S$. Compute the connected component $G^{0}$ of $G$ containing $w$, by walking through $G^{0}$. The time needed for doing so is exponential in $\ell(v)+\ell(w)$, since $\# G^{0} \leq \# G$ is exponential. By B.6.1, $v$ and $w$ are conjugate if and only if $v \in G^{0}$.

\section{References}

[1] J. M. Alonso et al., Notes on word hyperbolic groups (ed. by H. Short). In Group theory from a geometrical viewpoint (Trieste, 1990), World Scientific, Singapore 1991, 3-63. Zbl 0849.20023 MR 1170363

[2] U. Baumgartner, Totally disconnected, locally compact groups as geometric objects. A survey of work in progress. In Geometric group theory (Geneva and Barcelona, 2005). Trends Math., Birkhäuser Verlag, Basel 2007, 1-20. Zbl 05343228 MR 2395785

[3] U. Baumgartner, B. Rémy, and G. A. Willis, Flat rank of automorphism groups of buildings. Transform. Groups 12 (2007), 413-436. Zbl 1145.22012 MR 2356316

[4] A. F. Beardon, The geometry of discrete groups. Grad. Texts in Math. 91, Springer-Verlag, New York 1983. Zbl 0528.30001 MR 0698777

[5] R. Benedetti and C. Petronio, Lectures on hyperbolic geometry. Universitext, SpringerVerlag, Berlin 1992. Zbl 0768.51018 MR 1219310

[6] A. Berman and R. J. Plemmons, Nonnegative matrices in the mathematical sciences. Academic Press, New York 1979. Zbl 0484.15016 MR 0544666 
[7] A. Björner and F. Brenti, Combinatorics of Coxeter groups. Grad. Texts in Math. 231, Springer-Verlag, New York 2005. Zbl 1110.05001 MR 2133266

[8] N. Bourbaki, Groupes et algèbres de Lie, Ch. 4-6. Hermann, Paris 1968; Masson, Paris 1981. Zbl 0186.33001 MR 0240238

[9] M. R. Bridson and A. Haefliger, Metric spaces of non-positive curvature. Grundlehren Math. Wiss. 319, Springer-Verlag, Berlin 1999. Zbl 0988.53001 MR 1744486

[10] B. Brink and R. B. Howlett, A finiteness property and an automatic structure for Coxeter groups. Math. Ann. 296 (1993), 179-190. Zbl 0793.20036 MR 1213378

[11] A. Brønsted, An introduction to convex polytopes. Grad. Texts in Math. 90, SpringerVerlag, New York 1983. Zbl 0509.52001 MR 0683612

[12] K. S. Brown, Buildings. Springer Monogr. Math., Springer-Verlag, New York 1998. Zbl 0922.20034 MR 1644630

[13] P.-E. Caprace, Conjugacy of one-ended subgroups of Coxeter groups and parallel walls. Preprint 2005. arXiv:math/0508057

[14] P.-E. Caprace, Conjugacy of 2-spherical subgroups of Coxeter groups and parallel walls. Algebr. Geom. Topol. 6 (2006), 1987-2029. Zbl 05118585 MR 2263057

[15] P.-E. Caprace, Buildings with isolated subspaces and relatively hyperbolic Coxeter groups. Preprint 2007. arXiv:math/0703799

[16] P.-E. Caprace and F. Haglund, On geometric flats in the CAT(0) realization of Coxeter groups and Tits buildings. Canad. J. Math., to appear; Preprint 2006. arXiv:math/0607741

[17] P.-E. Caprace and B. Mühlherr, Reflection triangles in Coxeter groups and biautomaticity. J. Group Theory 8 (2005), 467-489. Zbl 1081.20049 MR 2152693

[18] P.-E. Caprace and B. Rémy, Simplicity and supperrigidity of twin building lattices. Invent. Math., to appear; Preprint 2006. arXiv:math/0607664

[19] A. M. Cohen, Coxeter groups and three related topics. In Generators and relations in groups and geometries, Castelvecchio Pascoli (Lucca), 1990, NATO Adv. Sci. Inst. Ser. C Math. Phys. Sci. 333, Kluwer Acad. Publ., Dordrecht 1991, 235-278. Zbl 0742.20040 MR 1206917

[20] A. M. Cohen, Recent results on Coxeter groups. In Polytopes: abstract, convex and computational (Scarborough, Ontario, 1993), NATO Adv. Sci. Inst. Ser. C Math. Phys. Sci. 440, Kluwer Acad. Publ., Dordrecht 1994, 1-19. Zbl 0830.20060 MR 1322055

[21] G. E. Collins and R. Loos, Real zeros of polynomials. In Computer algebra: symbolic and algebraic computation, Springer-Verlag, Vienna 1983, 83-94. Zbl 0533.68038 MR 0728967

[22] M. Coornaert, T. Delzant, and A. Papadopoulos, Géométrie et théorie des groupes. Lecture Notes in Math. 1441, Springer-Verlag, Berlin 1990 Zbl 0727.20018 MR 1075994

[23] H. S. M. Coxeter, The complete enumeration of finite groups of the form $R_{i}^{2}=$ $\left(R_{i} R_{j}\right)^{k_{i j}}=1$. J. London Math. Soc. 10(1935) 21-25. JFM 61.0097.05 Zbl 0010.34202

[24] J. H. Davenport, Y. Siret, and E. Tournier, Computer algebra. Academic Press, London 1988. Zbl 0679.68058 MR 0975254 
[25] M. W. Davis, The geometry and topology of Coxeter groups. London Math. Soc. Monogr. 32, Princeton University Press, Princeton, NJ, 2007. Zbl 1142.20020 MR 2360474

[26] M. Dehn, Über unendliche diskontinuierliche Gruppen. Math. Ann. 71 (1912), 116-144. JFM 42.0508.03

[27] V. V. Deodhar, On the root system of a Coxeter group. Comm. Algebra 10 (1982), 611-630. Zbl 0491.20032 MR 647210

[28] V. V. Deodhar, A note on subgroups generated by reflections in Coxeter groups. Arch. Math. (Basel) 53 (1989), 543-546. Zbl 0688.20028 MR 1023969

[29] S. van Dongen, Exact computation of the sign of a real algebraic number. Master's thesis, Technical University of Eindhoven, Eindhoven 1994.

[30] M. Dyer, Reflection subgroups of Coxeter systems. J. Algebra 135 (1990), 57-73. Zbl 0712.20026 MR 1076077

[31] D. B. A. Epstein, J. W. Cannon, D. F. Holt, S. V. F. Levy, M. S. Paterson, and W. P. Thurston, Word processing in groups. Jones and Bartlett Publishers, Boston 1992. Zbl 0764.20017 MR 1161694

[32] D. Epstein and D. Holt, The linearity of the conjugacy problem in word-hyperbolic groups. Internat. J. Algebra Comput. 16 (2006), 287-305. Zbl 1141.20028 MR 2228514

[33] P. de la Harpe, An invitation to Coxeter groups. In Group theory from a geometrical viewpoint (Trieste, 1990), World Scientific, Singapore 1991, 193-253. Zbl 0840.20033 MR 1170367

[34] D. F. Holt, B. Eick, and E. A. O'Brien, Handbook of computational group theory. Chapman \& Hall/CRC Press, Boca Raton 2005. Zbl 1091.20001 MR 2129747

[35] R. B. Howlett, Normalizers of parabolic subgroups of reflection groups. J. London Math. Soc. (2) 21 (1980), 62-80. Zbl 0427.20040 MR 576184

[36] J. E. Humphreys, Reflection groups and Coxeter groups. Cambridge Stud. Adv. Math. 29, Cambridge University Press, Cambridge 1990. Zbl 0725.20028 MR 1066460

[37] R. D. Hurwitz, A survey of the conjugacy problem. In Contributions to group theory, Contemp. Math. 33, Amer. Math. Soc., Providence, RI, 1984, 278-298. Zbl 0549.20023 MR 0767116

[38] N. Jacobson, Lectures in abstract algebra. III. Grad. Texts in Math. 32, Springer-Verlag, New York 1975. Zbl 0322.12001 MR 0392906

[39] D. E. Knuth, The art of computer programming. Vol. 2: Seminumerical algorithms, 2nd ed., Addison-Wesley Publishing Co., Reading, Mass., 1981. Zbl 0477.65002 MR 0633878

[40] Yu. I. Manin, A course in mathematical logic. Grad. Texts in Math. 53, Springer-Verlag, New York 1977. Zbl 0383.03002 MR 0457126

[41] G. Maxwell, Sphere packings and hyperbolic reflection groups. J. Algebra 79 (1982), 78-97. Zbl 0556.51003 MR 679972

[42] J. Milnor, A note on curvature and fundamental group. J. Differential Geometry 2 (1968), 1-7. Zbl 0162.25401 MR 0232311

[43] G. Moussong, Hyperbolic Coxeter groups. Ph.D. thesis, The Ohio State University, Columbus 1988. http://www.ohiolink.edu/etd/view.cgi?osu1114437114 
[44] L. Paris, Irreducible Coxeter groups. Internat. J. Algebra Comput. 17 (2007), 427-447. Zbl 1134.20046 MR 2333366

[45] L. Solomon, A Mackey formula in the group ring of a Coxeter group. J. Algebra 41 (1976), 255-264. Zbl 0355.20007 MR 0444756

[46] J. Tits, Le problème des mots dans les groupes de Coxeter. In Symposia Mathematica, vol. 1, Teoria dei gruppi, INDAM, Roma, 13-16 dicembre 1967, Academic Press, London 1969, 175-185. Zbl 0206.03002 MR 0254129

[47] È. B. Vinberg, Discrete linear groups that are generated by reflections. Izv. Akad. Nauk SSSR Ser. Mat. 35 (1971), 1072-1112; English transl. Math. USSR-Izv. 5 (1971), 10831119. Zbl 0247.20054 MR 0302779

[48] È. B. Vinberg, Hyperbolic groups of reflections. Uspekhi Mat. Nauk 40 (1985), 29-66; English transl. Russian Math. Surveys 40 (1985), 31-75. Zbl 0579.51015 MR 783604

Received September 13, 2007

D. Krammer, University of Warwick, Mathematics Department, Coventry CV4 7AL, United Kingdom

E-mail: D.Krammer@warwick.ac.uk 